\title{
Single Cell Enhancer Activity Maps Neuronal Lineages in
}

\section{Embryonic Mouse Basal Ganglia}

Linda Su-Feher ${ }^{1,2,12}$, Anna N. Rubin ${ }^{3,12}$, Shanni N. Silberberg ${ }^{3}$, Rinaldo Catta-Preta1,2,11,

6 Kenneth J. Lim ${ }^{1,2,3}$, Iva Zdilar,2, Christopher S. McGinnis ${ }^{4}$, Gabriel L. McKinsey ${ }^{5}$,

7 Thomas E. Rubino, Jr. ${ }^{1,2}$, Michael Hawrylycz ${ }^{6}$, Carol Thompson $^{6}, Z^{2}$ ev J. Gartner ${ }^{4,7,8,9}$,

10 1Department of Psychiatry and Behavioral Sciences, University of California, Davis,

11 Davis, California, USA. 2Department of Neurobiology, Physiology and Behavior,

12 University of California, Davis, Davis, California, USA. ${ }^{3}$ Nina Ireland Laboratory of

13 Developmental Neurobiology, Department of Psychiatry, University of California, San

14 Francisco Medical School, San Francisco, California, USA. ${ }^{4}$ Department of

15 Pharmaceutical Chemistry, University of California, San Francisco, San Francisco, CA,

16 USA. ${ }^{5}$ Department of Pediatrics, University of California, San Francisco, San Francisco,

17 USA. ${ }^{6}$ Allen Institute for Brain Science, Seattle, WA, USA. ${ }^{7}$ Helen Diller Family

18 Comprehensive Cancer Center, San Francisco, CA, USA. ${ }^{8}$ Chan Zuckerberg BioHub,

19 University of California San Francisco, San Francisco, CA, USA. ${ }^{9}$ Center for Cellular

20 Construction, University of California San Francisco, San Francisco, CA, USA.

$21{ }^{10}$ Department of Human Anatomy and Psychobiology and IMIB-Arrixaca Institute,

22 University of Murcia, Spain. ${ }^{11}$ Current address: Department of Genetics, Blavatnik 
23 Institute, Harvard Medical School, Boston, Massachusetts, USA. ${ }^{12}$ These authors

24 contributed equally.

25

26 \#Correspondence to Alex S. Nord (asnord@ucdavis.edu) and John L. R. Rubenstein

27 (john.rubenstein@ucsf.edu). 


\section{Abstract}

30 Enhancers integrate transcription factor signaling pathways that drive cell fate

31 specification in the developing brain. We used single cell RNA-sequencing (scRNA-seq)

32 to capture enhancer activity at single cell resolution and delineate specification of cells

33 labeled by enhancers in mouse medial, lateral, and caudal ganglionic eminences (MGE,

34 LGE, and CGE) at embryonic day (E)11.5. We combine enhancer-based reporter

35 labeling with single-cell transcriptional readout to characterize enhancer activity and

36 define cell populations in vivo. Seven enhancers had diverse activities in specific

37 progenitor and neuronal populations within the GEs. We then applied enhancer-based

38 labeling, scRNA-seq, and analysis of in situ hybridization (ISH) data to distinguish

39 subtypes of MGE-derived GABAergic and cholinergic projection neurons and

40 interneurons. This work demonstrates how the power of scRNA-seq can be extended by

41 enhancer-based labelling and leveraging ISH data and reveals novel lineage

42 specification paths underlying patterning of developing mouse brain. 


\section{Introduction}

45 During brain development, transcriptional programs governed by the genomic interplay

46 of transcription factors and cis-regulatory enhancer and promoter sequences drive the

47 proliferation and specification of neuronal and glial lineages (Beccari et al., 2013; Nord,

48 2015). An understanding of this regulatory symphony in the telencephalon has been

49 derived via decades of genetic dissection of transcription factor signaling (Kessaris et

50 al., 2014; Lim et al., 2018; Long et al., 2009), more recently extended via genomic

51 approaches (Lindtner et al., 2019; Sandberg et al., 2016), and is now undergoing a

52 revolution via application of single cell RNA-sequencing (scRNA-seq). scRNA-seq has

53 produced fine-scale elucidation of cell types in the mammalian brain (Zeisel et al.,

54 2018); however, major challenges remain towards understanding the dynamics of cell

55 state and identity that occur in the context of neurodevelopment.

57 The embryonic basal ganglia (BG) include spatially distinct proliferative zones of the

58 ganglionic eminences (GEs), which include the medial, lateral, and caudal ganglionic

59 eminences (MGE, LGE, and CGE) (J.L.R. and Campbell, 2020). Progenitor cells in the

60 ventricular (VZ) and subventricular (SVZ) domains in the GEs give rise to many

61 neuronal classes. Neuron types that originate in embryonic BG include GABAergic

62 projection neurons and cholinergic neurons (Fragkouli et al., 2009) that form the ventral

63 pallidum, globus pallidus (Flandin et al., 2010; Nóbrega-Pereira et al., 2010), and striatal

64 structures (J.L.R. and Campbell, 2020) that make up the mature BG. In addition, the

65 GEs generate interneurons that populate the striatum, cortex, olfactory bulb, and other 
66 brain regions (Anderson et al., 1997; Batista-Brito et al., 2020; Lim et al., 2018; Marín et

67 al., 2000). Building on bulk transcriptomics and in situ hybridization studies (ISH),

68 scRNA-seq has been applied to embryonic mouse BG, revealing generalized progenitor

69 populations and early born GABAergic lineages, with a focus on cortical interneuron

70 (CIN) specification (Mayer et al., 2018; Mi et al., 2018). While CINs are one major

71 output of embryonic BG, single cell characterization of GABAergic and cholinergic as

72 well as early born CIN lineages and that arise in the BG remains largely unexplored.

73 Resolving the early stages of BG neurogenesis via scRNA-seq and ISH has been

74 limited by major barriers: paucity of region- and lineage-specific single gene markers,

75 similarity of early transcriptional programs, spatial mixing of progenitors within germinal

76 zones and immature cell types in the $M Z$, and regional organization of $B G$ neurogenesis

77 that has been poorly captured by unguided scRNA-seq analysis.

79 Fate mapping via reporter labeling has provided critical insights into the origins of

80 neuronal cell populations (Batista-Brito et al., 2020). Notably, enhancers drive highly

81 specific transcription pattens, including in the developing telencephalon (Visel et al.,

82 2013), thus offering exciting possibilities for cell-type specific labeling and genetic

83 manipulation. We previously demonstrated the utility of enhancer-driven transgenic

84 reporter mouse lines for fate mapping and genetic manipulation of neuronal populations

85 originating in embryonic BG and cortex (Pattabiraman et al., 2014; Silberberg et al.,

86 2016). We generated transgenic mice harboring evolutionarily conserved enhancer

87 sequences that drive expression of $\mathrm{CreER}^{\mathrm{T} 2}$ and GFP. These developmental enhancers

88 exhibited spatiotemporal activity across expression domains within the embryonic BG 
89 and mark early cell populations prior to terminal cell fate commitment. Enhancers

90 differentially labeled cell populations that spatially intermingle and alternatively marked

91 regionally distinct mitotic and postmitotic populations during development. Fate mapping

92 with these enhancer-driven CreER ${ }^{\mathrm{T} 2}$-GFP mice demonstrated that developmental

93 lineages marked by transient enhancer activity produce varied mature neuron

94 populations within and across these enhancers. Importantly, beyond their use in

95 understanding neuronal lineages, these enhancer-driven reporter lines offer the

96 opportunity for function-based analysis of dynamic in vivo enhancer activity, a missing

97 feature from studies modeling enhancer activity via epigenomic approaches. More

98 broadly, enhancer-based cell labeling is emerging as a powerful tool for cell-type

99 identification, enrichment, and modulation in neuroscience and other areas, yet little

100 remains known about sensitivity and specificity of enhancer-driven reporter expression

101 at single cell resolution.

102

103 In this study, we apply the novel strategy of pairing enhancer-based transgenic reporter

104 mouse lines with scRNA-seq to define specific enhancer-labeled lineages at single cell

105 resolution in early embryonic BG. These experiments reveal functionally defined distinct

106 enhancer activities across scRNA-seq-defined cell states and lineages. Next, we

107 focused on the MGE and integrated ISH mapping of transcript expression from the Allen

108 Developing Mouse Brain Atlas (ABA) (Lein et al., 2007) to provide a higher resolution

109 anatomical definition of lineages identified by enhancer-labeling and scRNA-seq. Our

110 study identified proliferative and postmitotic cells that are distinctly labeled by enhancers 
111 active in MGE, LGE, and CGE and revealed novel specification paths for enhancer-

112 labeled and spatially defined populations of early BG-derived neuronal lineages.

114 Results

116 Comparative activity of seven enhancers in E11.5 BG via scRNA-seq

118 We profiled enhancer-labeled cell populations from day (E)11.5 MGE, LGE, or CGE

119 across seven subpallial enhancer transgenic mouse lines (Silberberg et al., 2016)

120 (Figure 1A). The selected transgenic lines express GFP and CreER ${ }^{\top 2}$ with enhancer-

121 driven divergent patterns in the ventricular (VZ), subventricular (SVZ), and mantle (MZ)

122 zones of the GEs. These enhancers are putatively associated with developmentally

123 expressed genes and have restricted regional activity within the GEs at E11.5,

124 summarized in Figure 1B. The objective of these experiments was threefold. First, to

125 establish the utility and sensitivity to detect enhancer-driven reporter expression via

126 scRNA-seq. Second, to define and compare representative enhancer activities and

127 enhancer-labeled progenitors and early neuronal populations across MGE (enhancers

$128 h s 1538, h s 1056, h s 799$, and $h s 192)$, LGEs ( $h s 841$ and $h s 599)$, and CGE (hs841 and

$129 h s 953)$ at E11.5. Third, to resolve fine-scale differences among cells labeled by

130 regionally distinct MGE progenitor-associated enhancers ( $h s 1538$ and $h s 1056$ ) and

131 early neuronal enhancers that differentially label emerging lineages ( $h s 799$ and $h s 192$ ). 
A

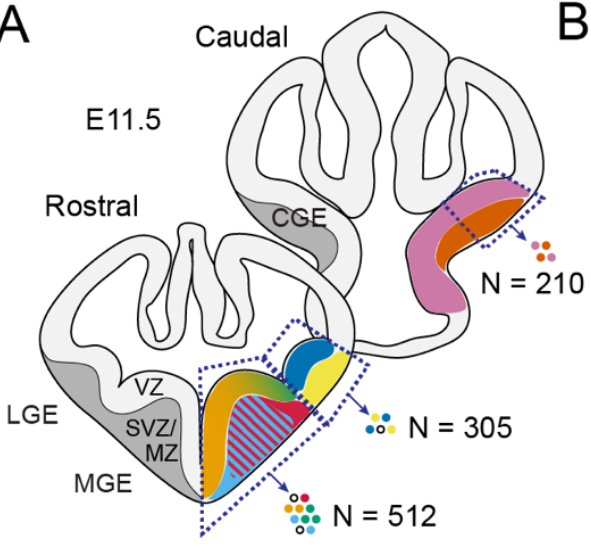

C Transgene Expression

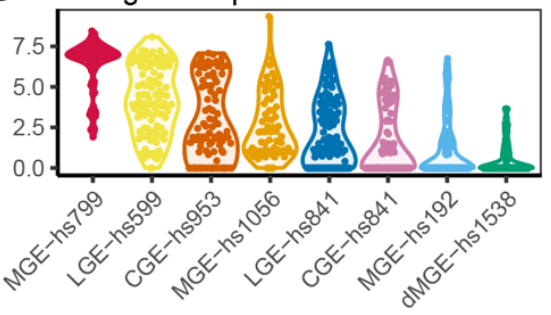

D

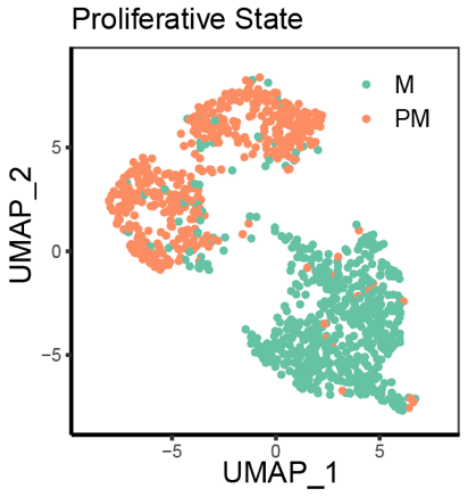

B

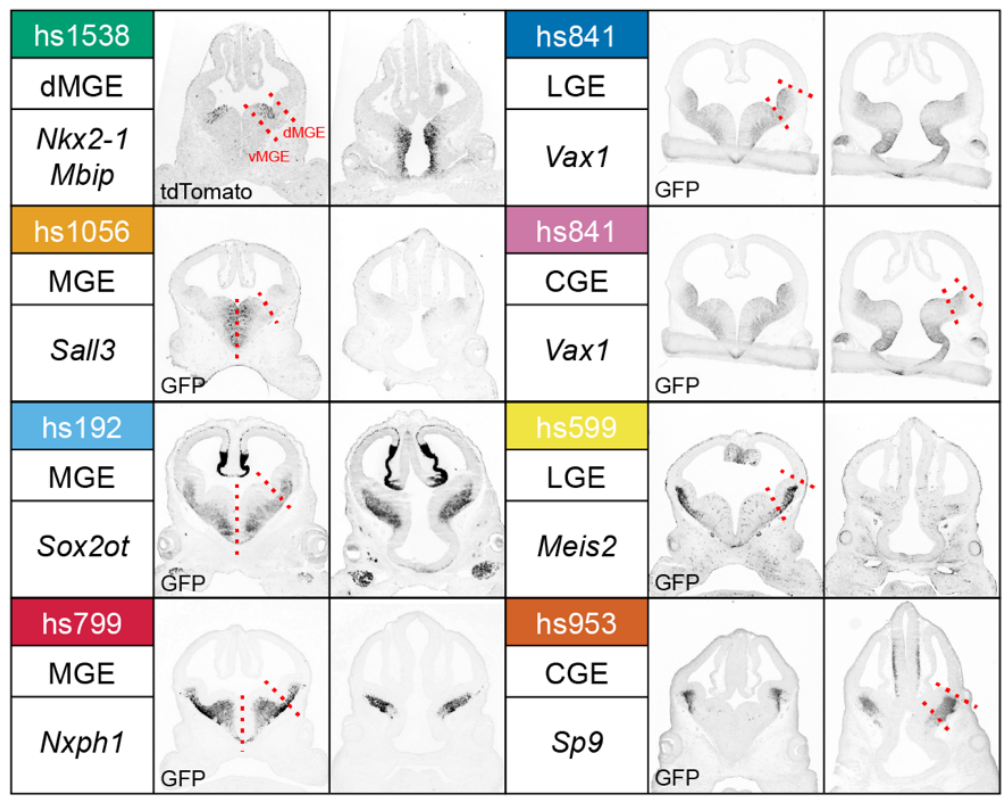

$\mathrm{F}$

Enhancer Group

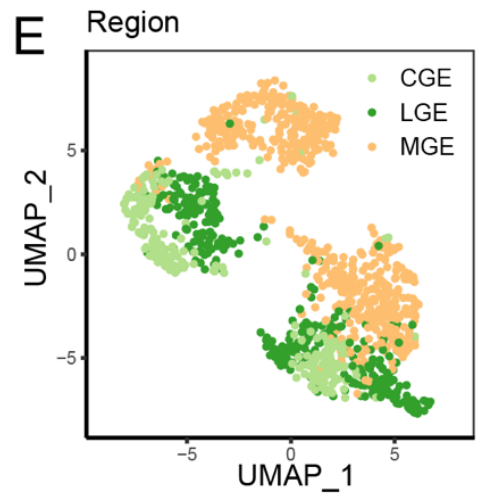

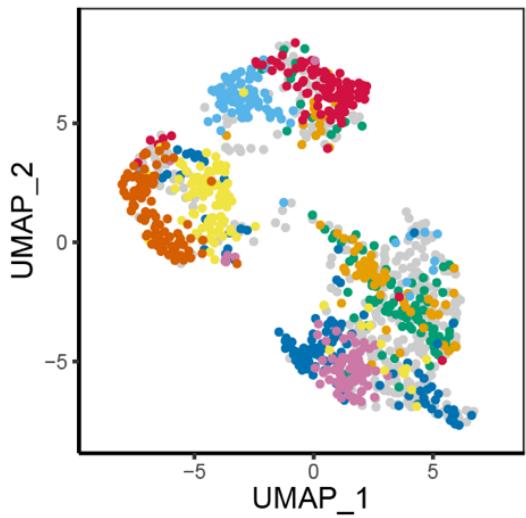

Figure 1: Profiling enhancer-labeled single cells in E11.5 basal ganglia.

(A) Schematic of dissection, with colors representing the activity of seven transgenic enhancer reporters characterized using C1 scRNA-seq. CGE: caudal ganglionic eminence; LGE: lateral ganglionic eminence; MGE: medial ganglionic eminence; VZ: ventricular zone; SVZ: subventricular zone; MZ: mantle zone. (B) Summary of the seven enhancers profiled, including dissection region, putative gene regulatory target, and representative GFP immunohistochemistry (IHC) imaging of enhancer transgenic reporters at E11.5, depicting activity within the ganglionic eminences. Red lines indicate microdissection boundaries. GFP IHC images are adapted from (Silberberg et al., 2016). (C) Violin plot of normalized transgene expression by enhancer group. (D) Visualization of single cells by UMAP, colored by mitotic state (green: M, mitotic; orange: PM, postmitotic). (E) Visualization of single cells by UMAP, colored by region of dissection (light orange: MGE; light green: CGE; dark green: LGE). (F) Visualization of single cells by UMAP, colored by transgenic enhancer grouping. Colors correspond to header colors in (B). Enhancer-negative cells are depicted in grey. 
134 Using the seven transgenic lines, we performed targeted BG microdissection and

135 preparation of reporter-positive and ungated single cells. Single cell suspensions were

136 either first segregated for transgene expression through fluorescence activated cell

137 sorting (FACS, Figure S1) or passed directly to the Fluidigm C1 system for capture and

138 amplification of the transcriptomes of individual cells. The regional dissections included:

139 for $h s 599$ the LGE; for $h s 953$ the CGE; for $h s 1538$ the dorsal (d)MGE; and for $h s 1056$,

$140 h s 192$, and $h s 799$, the MGE. For one enhancer, $h s 841$, we independently dissected the

141 LGE and CGE. For $h s 1538$, we used CreER ${ }^{\mathrm{T2}}$-driven tdTomato signal via cross to Ai14

142 reporter mice (Madisen et al., 2010) for gating due to low GFP signal. For details

143 regarding sample preparation, see Table S1. After sequencing and quality control

144 (Figure S2A-L), 1027 cells were included for analysis, with $\sim 594,000$ reads and $\sim 5,140$

145 genes per cell on average.

147 Our first objective was to demonstrate the feasibility of single cell enhancer activity

148 mapping by establishing whether enhancer-driven transgene expression could be

149 mapped to single cells via scRNA-seq. We used a combination of $\mathrm{FACS}^{+}$gating and

150 transgene (CreER ${ }^{\text {T2}}$-IRES-GFP or tdTomato) RNA expression to assign cells as

151 enhancer-positive or negative ("None"). 315 cells were unsorted or FACS" and

152 transgene negative; 712 cells were $\mathrm{FACS}^{+}$and/or expressed non-zero transgene.

153 Based on tissue dissection and enhancer line, enhancer-positive cells were assigned to

154 one of eight categories: MGE-hs1056, MGE-hs1538, MGE-hs192, MGE-hs799, LGE-

155 hs599, CGE-hs953, LGE-hs841, and CGE-hs841. Enhancer-labeled cells generally

156 exhibited expression of presumed target genes with some exceptions. Enhancer-driven 
157 reporter transcripts were detectable at single cell resolution across all enhancers, with

158 variation in presence and transcript level captured via scRNA-seq (Figure 1C). Five of

159 the seven enhancers exhibited strong concordance between reporter protein GFP+

160 gating and transgene transcript detected via scRNA-seq (Figure S2M). The other two

161 lines, dMGE-hs1538 and MGE-hs192, had weaker sensitivity, with 30-40\% of FACS

162 reporter-positive cells having detectable transgene transcript. Nonetheless, even for

163 enhancers with weaker transcriptional activity, reporter transgene was reliably detected

164 via scRNA-seq in a substantial fraction of FACS-determined reporter-positive cells,

165 demonstrating the overall utility of this approach for function-based scRNA-seq

166 enhancer activity profiling.

TF expression organizes scRNA-seq data by proliferative state and BG region

170 Using highly variable genes in scRNA-seq analysis is a common approach for feature

171 selection (Butler et al., 2018); however, this method did not adequately separate

172 regional and cell state identity in our data (Figure S3). As an alternative, we used a

173 transcription factor (TF)-curated approach, with the rationale that TFs drive lineage

174 specification and cell identity. We rooted this analysis using 689 TFs profiled for RNA

175 ISH patterns at E11.5 and E13.5 in the Allen Developing Mouse Brain Atlas (ABA) (Lein

176 et al., 2007) (Table S2, Figure S4A-B). 455 of these TFs were expressed in our E11.5

177 scRNA-seq data, of which $292(64.2 \%)$ had detectable ISH expression in the BG

178 (Figure S4C-E). We used these 455 TFs to define scRNA-seq cell identity and for visual

179 representation via UMAP plots (Figure 1D-F). Using this TF-curated approach, 
180 proliferative state and regional origin were the primary aspects of scRNA-seq variation

181 (Figures 1D-E). We compared TF-curated analysis to results using highly variable

182 genes (Figures S3A-C), before and after performing regression analysis to reduce the

183 influence of cell cycle phase (Figures S3D-F). Excluding non-TFs reduced the

184 contribution of cell cycle phase and confounding sources of variation (e.g. sequencing

185 batch) to cell clustering and improved separation by GE origin (Figure S3G-I).

187 Enhancers label cells with specific regional identities and developmental

188 trajectories

190 Having shown that scRNA-seq can reliably identify enhancer-positive cells and that our

191 TF-curated approach enables separation across regional origin and proliferative states,

192 we next modeled transcriptional differences across enhancer-positive cells. First, we

193 modeled transitional cell states via diffusion mapping. Second, we examined differences

194 across cluster-based transcriptional identities. We identified two major diffusion

195 components (DC) corresponding to proliferative state (DC1) and MGE from LGE/CGE

196 origin (DC2) (Figure 2A-F). DC1 captured the stem cell, proneural, and neurogenic

197 transition, with genes such as Hes1, Ccnd2, Gadd45g, and S/c34a2 marking cells at

198 various stages of this transition (Figure 2G). Lower values of DC2 were associated with

199 MGE identity, marked by expression such MGE-specific genes such as Nkx2-1, Lhx6,

200 and Lhx8 (Flames et al., 2007) (Figure 2G). Higher values of DC2 were associated with

201 LGE or CGE identity (Figure 2D,F). DC2 diversity was driven by expression of region-

202 defining TFs such as Nkx2-1 in the MGE and Pax6 in the CGE and LGE (Figure 2G,I). 

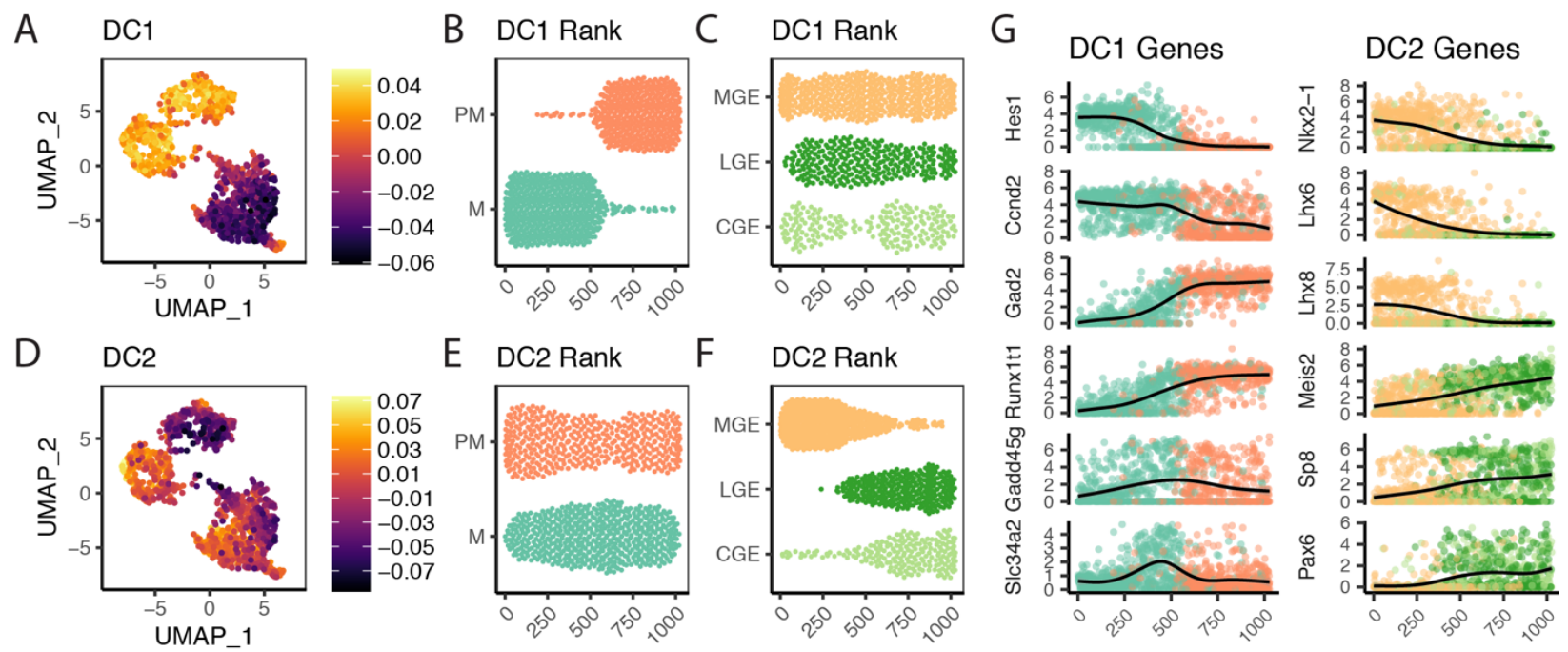

$\mathrm{H}$

I

M PM

GE Markers

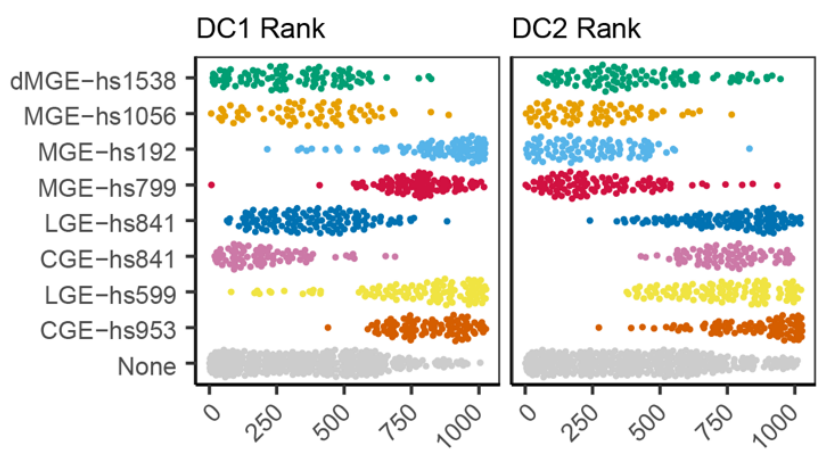

dMGE-hs1538 MGE-hs1056

MGE-hs192

$\Omega \Omega \Omega \Omega \Omega \Omega+1 \Omega \Omega 1$ MGE-hs799 LGE-hs841

CGE-hs841

LGE-hs599

CGE-hs953
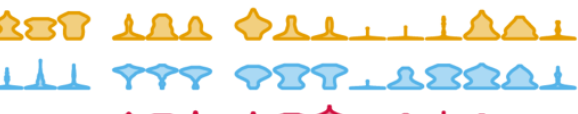

None

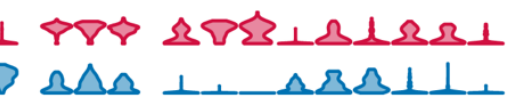

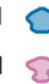

$\Omega \Omega \Omega$

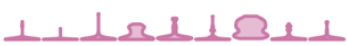

1

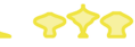

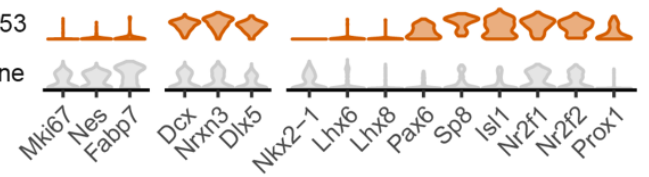

Figure 2: Diffusion mapping reveals progenitor state and regional identity gradients.

$(A, D)$ UMAP colored by diffusion component value (DC)1 (A) or DC2 (D). (B,E) Cells ranked by DC1 (B) or DC2 (E) value, separated and colored by mitotic state. (C,F) Cells ranked by DC1 (B) or DC2 (E) value, separated and colored by ganglionic eminence. (G) Relative expression of differentially expressed genes across DC1 (left) or DC2 (right). Cells on x-axis are ordered by DC1 or DC2 rank. Line represents generalized additive model (gam) line. (H) Cells ranked by DC1 (left) or DC2 (right) rank, separated and colored by enhancer group. (I) Violin plots of relative expression by enhancer group for marker genes associated with mitotic identity (M), postmitotic identity (PM), and various markers with ganglionic eminence-associated expression.

203 The strongest separation of LGE and CGE identity are the caudal-biased TFs Nr2f1 and

204 Nr2f2 (Hu et al., 2017) (Figure 2I). DC1 and DC2 values distinguished cells labeled by

205 different enhancers and indicate that these developmental enhancers are active across

206 maturation states within the GEs (Figure 2H). VZ-associated enhancers dMGE-hs1538,

207 MGE-hs1056, and CGE- and LGE-hs841 labeled cells across the proliferative zone of 
208 DC1, indicating enhancer activity across multiple maturation states (Figure $2 \mathrm{H}$ ). In 209 contrast, SVZ/MZ-associated enhancers MGE-hs192, MGE-hs799, LGE-hs599, and

210 CGE-hs953 labeled cells across the postmitotic zone of DC1, indicating these

211 enhancers are active across neuronal maturation (Figure $2 \mathrm{H}$ ).

213 We next performed clustering using TF-curated scRNA-seq expression, identifying 12

214 cell clusters that separated by proliferative state and regional or cell-type identity (Figure

$2153 \mathrm{~A}$, Table S3). We further used random forest classification to define informative

216 transcripts that discriminate cells labelled by specific enhancers (Table S4). Cells

217 labeled by enhancers dMGE-hs1538, MGE-hs1056, CGE-hs841 and LGE-hs841

218 primarily grouped into mitotic clusters $(\mathrm{cl})-1, \mathrm{cl}-2, \mathrm{cl}-3, \mathrm{cl}-8$, and $\mathrm{cl}-9$, further separated

219 by regional identity (MGE versus non-MGE). Within these regional boundaries, VZ/SVZ-

220 associated enhancers split across multiple clusters (Figure 3B), paralleling diffusion

221 mapping results suggesting mitotic enhancers label multiple proliferative states.

222 Compared to enhancers with progenitor activity, enhancers active in postmitotic cells

223 (MGE-hs192, MGE-hs799, LGE-hs599, and CGE-hs953) were biased toward specific

224 cell type clusters within broader regional identities (Figure 3B). To characterize cell

225 types that were differentially labeled by these enhancers, we performed differential gene

226 expression analysis using the full transcriptome of 17,015 expressed genes to identify

227 differentially expressed (DE) genes for each TF-defined cluster (Figure 3C-E). 

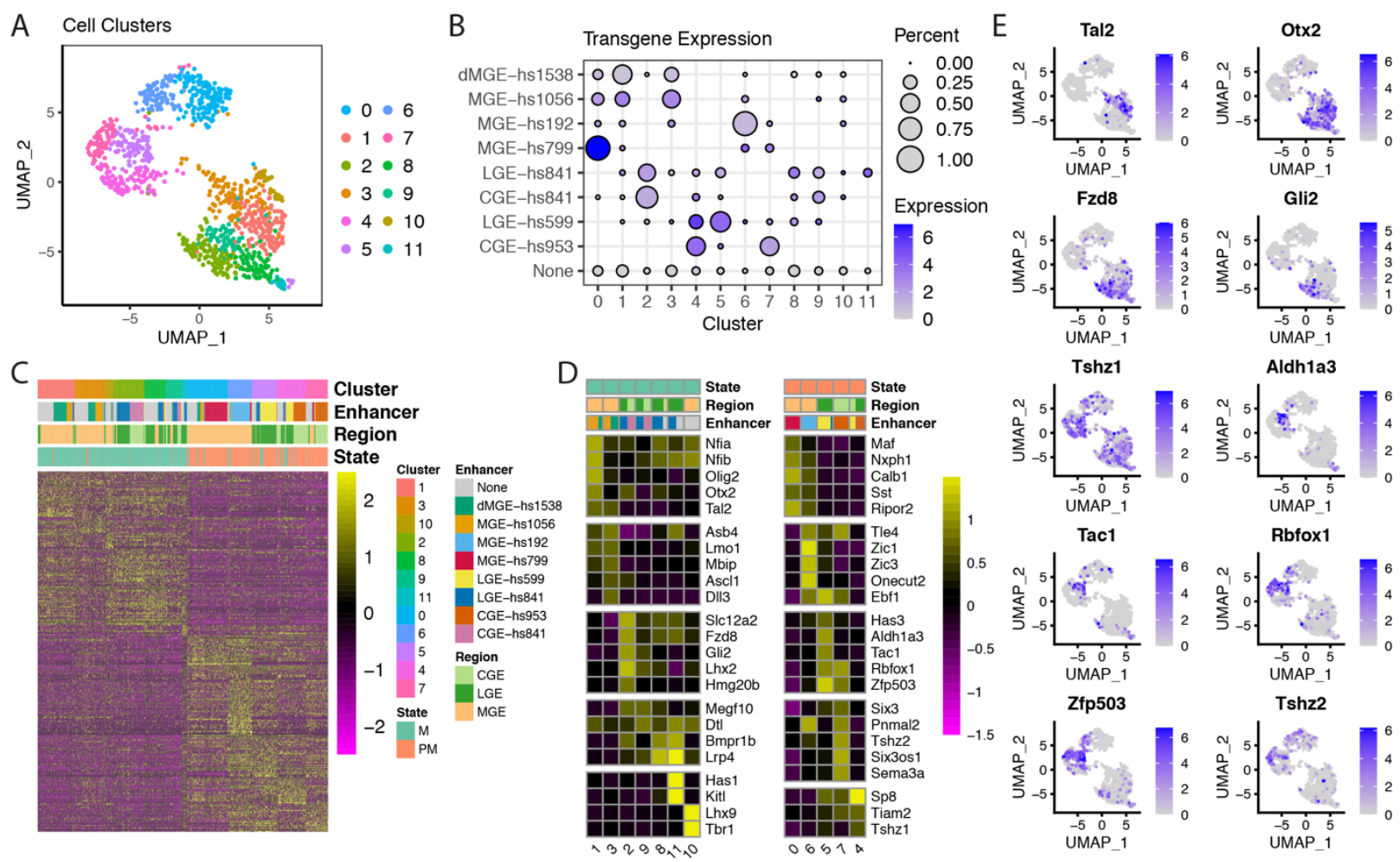

Tac1
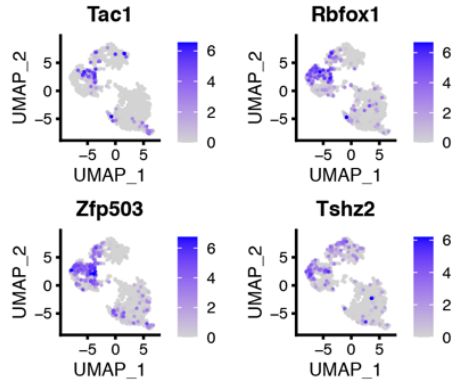

Figure 3: Enhancers label distinct transcriptional signatures.

(A) UMAP colored by TF-defined cell cluster. (B) Dot plot of relative transgene expression and representation of each enhancer across clusters. Size of dot represents percent of enhancer corresponding to a specific cluster. Color gradient represents mean normalized transgene expression for each enhancer by cluster. (C) Ordered heatmap of differentially expressed genes, in order of cluster, enhancer, regional origin, and proliferative state. Color gradient represents Z-score of normalized expression. (D) Representative differentially expressed genes by cluster. Color represents the mean Zscore of normalized expression across clusters. Each column color bar represents the proportion representation of proliferative state, region, or enhancer group. (E) UMAP plots of representative genes from (D), colored by relative expression.

Proliferative clusters $\mathrm{cl}-1,3,2$, and 9 encompassed proliferating cells including those

230 labeled by VZ/SVZ-associated enhancers MGE-hs1538, MGE-hs1056, and CGE- and

231 LGE-hs841. The mitotic-associated enhancers captured both early VZ and SVZ cells.

232 Cells in cl-1 expressed neural stem cell markers including higher expression of Nfia,

$233 \mathrm{Nfib}$, and Olig2 (Figure 3D). Cl-3 was associated with higher levels of intermediate

234 progenitor markers such as Asb4 and Asc/1. Proliferative LGE and CGE cells from 
236 and Fzd8. Enhancer-negative LGE and LGE-hs841 mitotic cells additionally form

237 clusters $\mathrm{Cl}-8$ and $\mathrm{Cl}-11$, which expressed genes such as Lrp4, Has1, and Kitl. $\mathrm{Cl}-10$ was

238 composed primarily of enhancer-negative MGE cells and expressed $L h \times 9$ and $T b r 1$,

239 indicative of cortical or diencephalic rather than basal ganglia identity. From random

240 forest classification, markers that distinguished LGE, CGE, and MGE progenitor-

241 associated enhancers recapitulated region-associated TFs from DC2 (Table S4). MGE

242 rostrodorsal ( $h s 1538)$ and caudoventral ( $h s 1056)$ biased enhancers were distinguished

243 by quantitative differences across TFs including Otx2 and Id4, identifying TF expression

244 gradients that distinguished progenitor cells across MGE regional axes.

246 Compared to proliferative enhancers, postmitotic enhancers active in SVZ/MZ mapped

247 to distinct transcriptional clusters corresponding to emerging neuron types. LGE-hs599

248 and CGE-hs953 are both represented in cl-4, which expressed higher levels of genes

249 including Sp8, Tiam2, and Tshz1 (Figure 3D-E), suggesting cl-4 is more immature than

$250 \mathrm{cl}-5$ and $\mathrm{cl}-7$ and has not yet acquired strong LGE or CGE regional specificity. Cl-5,

251 composed predominantly of LGE-hs599 cells, expressed genes including Rbfox1, Tac1,

252 and Zfp503. Cl-7, composed predominantly of CGE-hs953 and enhancer-negative cells,

253 expressed genes such as Six3, Tshz2, and Sema3a. Genes defining these clusters

254 shared general markers of early GABAergic projection neurons and were consistent

255 with fate mapping of $h s 599^{+}$and $h s 953^{+}$cells to projection neuron populations in the

256 adult forebrain, including striatal medium spiny neurons (McGregor et al., 2019;

257 Silberberg et al., 2016) and Sp8 $8^{+}$neurons in the amygdala (Silberberg et al., 2016).

258 MGE MZ-associated enhancers $h s 192$ and $h s 799$ also exhibited markers of early 
259 neuronal fate commitment (Figure 3D, Table S4). Cl-0, composed predominantly of

$260 h s 799^{+}$cells, expressed early MGE-derived cortical interneuron lineage markers

261 including Maf, Mafb, Nxph1, Calb1, and Sst. Conversely, cl-6, composed of primarily

262 hs $192^{+}$cells, expressed a wide range of TFs including Tle4 and Zic1, Zic3, and Zic4,

263 suggestive of GABAergic and cholinergic projection neuron commitment (Chen et al.,

264 2010).

266 These experiments captured the cell-type specific activity of seven evolutionarily

267 conserved enhancers across E11.5 GEs, showing the feasibility of enhancer-based

268 genetic labeling paired with single cell transcriptomics and resolving lineage and spatial

269 relationships via enhancer labeling. We found regionally separated but otherwise similar

270 mitotic identities among enhancer-labeled progenitors across MGE, LGE, and CGE, and

271 localized regional signatures within MGE via comparing dorsal $\left(h s 1538^{+}\right)$with more

272 ventral $h s 1056^{+}$cells. Across GEs, enhancer-labeled neuronal cell types emerged from

273 more general early postmitotic clusters. Postmitotic cells in MGE separated into

274 signatures suggesting GABAergic projection, cholinergic, and interneuron lineages

275 differentially labeled by $h s 192$ and $h s 799$. This initial survey defined specific cell

276 populations and identified known and novel markers for maturation state and regional

277 identity for progenitor and early born neuronal cell types, but had limited resolution to

278 capture heterogeneity within enhancer-labeled cells or profile enhancer-labeled

279 populations against all other cell types. 
281 Dissection of early born neuron types and positional identity in MGE via

282 enhancer-labeled 3' scRNA-seq and ISH

284 To more deeply interrogate emerging neuronal types labeled by $h s 192$ and $h s 799$ in

285 E11.5 MGE, we performed scRNA-seq using the 10x Genomics Chromium system. Our

286 initial scRNA-seq analysis of cells labeled by $h s 192$ and $h s 799$ suggested heterogeneity

287 within these labeled early neuronal populations in the MGE. Indeed, fate-mapping

288 experiments for these two enhancers (Silberberg et al., 2016) labeled other, MGE-

289 derived GABAergic, cholinergic, and interneuron populations as well as early born CINs,

290 populations that have not been the focus of previous scRNA-seq studies. FACS-purified

291 MGE ungated and reporter-positive cells were dissected from embryos across three

292 litters for each transgenic line and prepared for multiplexed scRNA-seq using MULTI-

293 seq (McGinnis et al., 2019) (Figure 4A, Table S1). After quality control (Figure S5A-B),

2944,001 single cells were used for downstream analysis. We used the same TF-curated

295 approach developed for the C1 scRNA-seq dataset to drive cell clustering. Of the 463

296 TFs detected in 10x scRNA-seq MGE data, 421 (90.9\%) were represented in both

297 Fluidigm C1 and 10x Chromium systems (Figure 4B, S4F-I). We identified 18 cell

298 clusters in the 10x dataset (Figure 4C). The largest determinant of transcriptional

299 variation across cells was proliferative state (Figure 4D). Consistent with the C1 data,

300 using TFs to drive cell clustering improved performance relative to highly variable gene

301 approaches (Figure S5C-E). Overall, the 10x and MGE-derived C1 data had similar

302 cluster topology and identified similar cell states, as determined by canonical correlation

303 analysis of both datasets (Figure S6). 
A

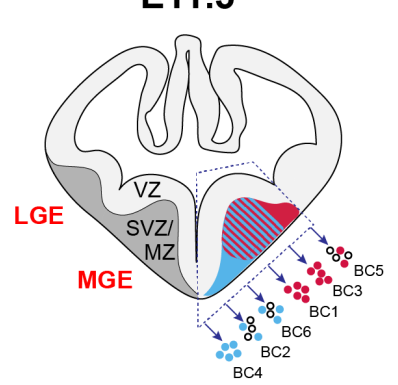

$\mathrm{E}$

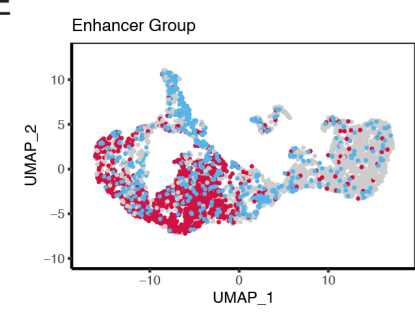

MGE-hs192 • MGE-hs799 — None

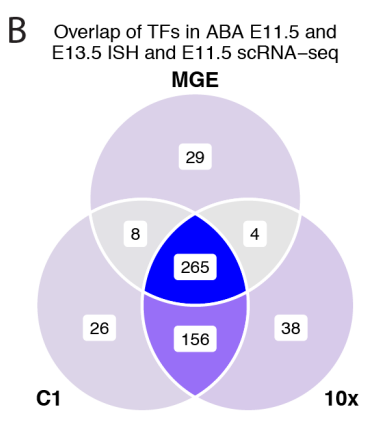

$\mathrm{F}$

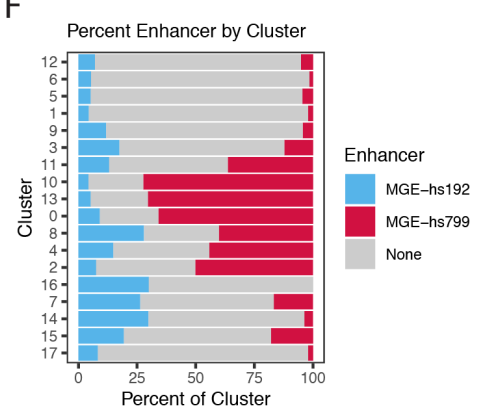

$\mathrm{H}$

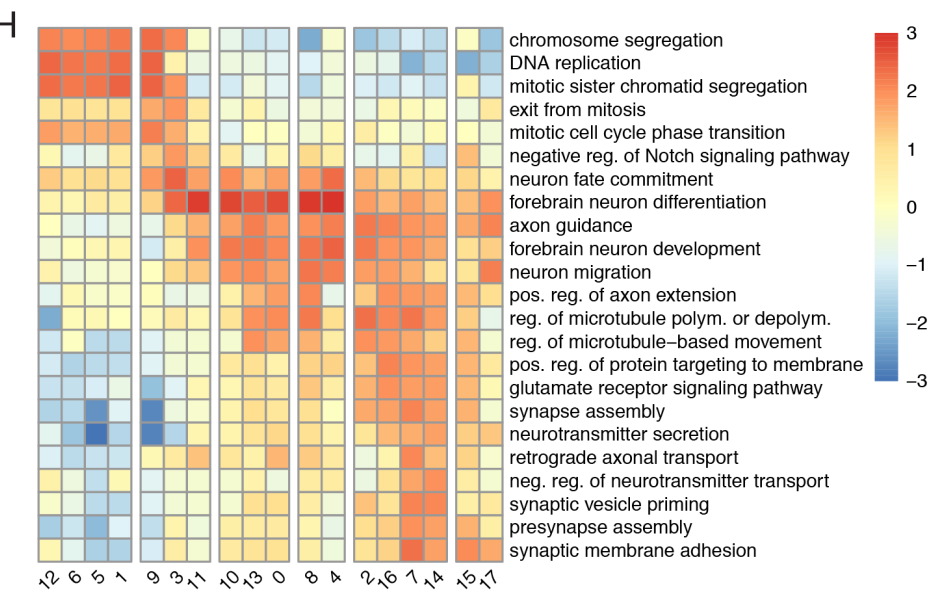

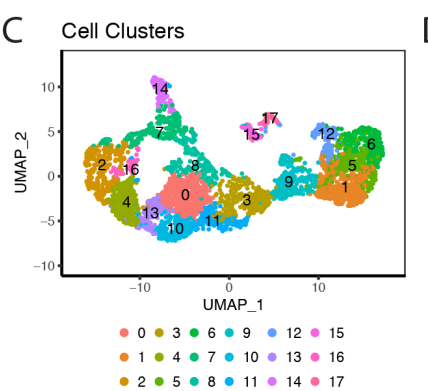

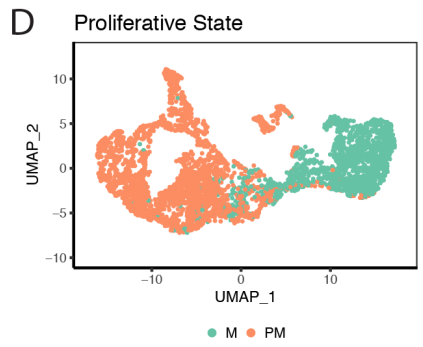

G
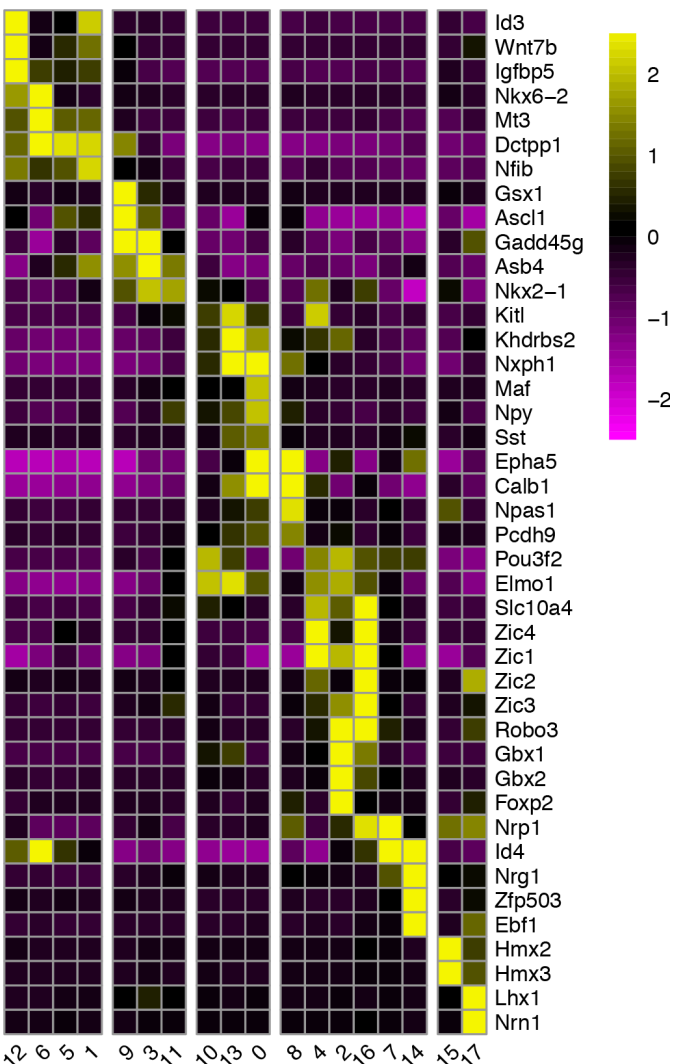

Figure 4: Regional and proliferative gradients within E11.5 MGE.

(A) Schematic of dissections used for MULTI-seq (McGinnis et al., 2019), with colors corresponding to enhancer transgenic reporter expression in the medical ganglionic eminence (red: $h s 799$; blue: $h s 192$; open circles: unlabeled). LGE: lateral ganglionic eminence; MGE: medial ganglionic eminence; VZ: ventricular zone; SVZ: subventricular zone; BC: barcode. (B) Venn diagram depicting overlap of transcription factors scored within the Allen Developing Mouse Brain Atlas (ABA) for expression in E11.5 or E13.5 basal ganglia and transcription factors detected in 10x and C1 scRNA-seq datasets. (C) UMAP colored by TF-anchored cell clusters. (D) Visualization of single cells from E11.5 MGE by UMAP, colored by mitotic state (green: M, mitotic; orange: PM, postmitotic). (E) UMAP colored by enhancer (red: $h s 799$; blue: hs192; grey: enhancer-negative). (F) Proportion representation of $h s 192$, hs799, and enhancernegative by cluster. (G) Representative differentially expressed genes by cluster. Color represents the mean Z-score of normalized expression across clusters. $(\mathbf{H})$ Representative gene ontology biological process terms separating clusters. Color represents $\log _{2}$ (observed/expected) for each term. 
305 Cells were defined as enhancer-positive for $h s 192$ or $h s 799$ if they had at least one UMI

306 (unique molecular identifier) count for CreER ${ }^{T 2}$-IRES-GFP. Enhancer-positive cells from

307 independent samples clustered together, demonstrating reproducibility across biological

308 replicates and distinct distributions of enhancer-labeled hs192 versus $h s 799$ cells

309 (Figure 4E). The majority of enhancer-labeled cells mapped to post-mitotic clusters.

$310 h s 799^{+}$cells were substantially enriched ( $>50 \%$ of cluster composition) in cl- 0,10 , and

31113 , and to a lesser extent in cl-2, 4, 8, and 11 (Figure 4F). In contrast, $h s 192^{+}$cells were

312 more broadly distributed, with the greatest representation in postmitotic clusters $\mathrm{cl}-7,8$,

31314,15 , and 16, and decreased representation in cl-0, 2, 10, and 13 (Figure 4F). Cells

314 labeled by at least one of the two enhancers were present in all postmitotic clusters

315 alongside ungated enhancer negative cells.

317 We next performed differential gene expression (DE) analysis and Gene Ontology

318 analysis across clusters using the full transcriptome of 18,088 genes to identify markers

319 for each cluster and resolve maturation states and cell type identities (Figure 4G-H,

320 Tables S3, S5). Proliferative clusters (cl-1, 5, 6, and 12), corresponding to cells within

321 the MGE VZ, were enriched in terms including 'DNA replication' and 'chromosome

322 segregation' (Figure 4H). In comparison, proliferative clusters $\mathrm{cl}-9$ and $\mathrm{cl}-3$, likely

323 corresponding to cells within the SVZ, were enriched for terms such as 'exit from

324 mitosis' (Figure 4H). Single cell resolution captured initiation of enhancer activity, which

325 is first evident for both $h s 799$ and $h s 192$ among individual cells in late SVZ clusters

326 based on transgene expression (Figures 4D-E). Postmitotic clusters subdivided into

327 MGE-derived maturing GABAergic and cholinergic neuron and interneuron lineages (cl- 
$0,2,4,7,8,10,11,13$, and 16), which are differentially labeled by $h s 192$ and $h s 799$,

329 and are described in detail below.

331 Some clusters were made up of postmitotic cells that appeared to originate outside the

332 MGE (cl-14, 15, and 17), and were likely migrating through the MGE at E11.5 or

333 captured at dissection boundaries. Cl-14 cells were enriched for $h s 192^{+}$cells and had

334 properties of LGE-derived immature medium spiny neurons, which express Ebf1, Nrg1

335 and Zfp503 (Nolz1), but not Nkx2-1 (Figure 4G). Cl-15 contained both $h s 192^{+}$and

$336 h s 799^{+}$cells and may derive from preoptic area (POA), based on Hmx2 (Nkx5-2) and

$337 \mathrm{Hm} \times 3(N k \times 5-1)$ expression. $\mathrm{Cl}-17$ cells were mostly transgene-negative, and may

338 originate from regions adjacent to the subpallium, e.g. from the hypothalamus or

339 prethalamic eminence. These non-MGE clusters are not further discussed.

341 Projecting scRNA-seq cell identities onto developing mouse MGE via ISH data

343 Using transcriptional cell identities defined by scRNA-seq, we applied ABA ISH

344 expression data (Lein et al., 2007) from the 689 TFs to map the anatomical distribution

345 of cell populations in the E11.5 telencephalon. First, we manually graded expression of

346 curated TFs in the VZ, SVZ, and MZ of MGE and/or LGE by reviewing individual

347 sections from E11.5 and E13.5 in the ABA (Table S2). From this analysis we identified

348332 genes with visually detectable mRNA ISH patterns in the BG, of which 283 were

349 also detected in E11.5 scRNA-seq from C1 and 10x (Figure S4C). Following

350 comprehensive review of genes with differential scRNA-seq expression and ABA ISH 
351 expression patterns in E11.5, E13.5, and E15.5 BG, we identified sentinel genes that

352 could be used as spatiotemporal markers to presumptively assign cells or clusters to

353 distinct neuroanatomical regions and/or specific neuronal lineages on the basis of their

354 mRNA expression profiles. Using these sentinel markers and our scRNA-seq data, we

355 characterized emergent cell lineages and regional distributions of proliferative (Figure 5)

356 and postmitotic (Figure 6) cell populations in the MGE.

MGE progenitors stratify by VZ to SVZ and dorsoventral and rostrocaudal axes

360 The analysis of mitotically active cells (Mki67+; Figure 5A) in the developing MGE

361 provided evidence for distinct progenitor stages and regional patterning (Figure 5B-C).

362 In our analysis, the assignment of four mitotic clusters was driven by genes previously

363 associated with progenitor cell maturation steps, suggesting four discrete histogenetic

364 stages: VZ1 (neuroepithelium), VZ2 (radial glial), SVZ1 (secondary progenitor 1), and

365 SVZ2 (secondary progenitor 2) (Figure 5A). This interpretation is supported by mRNA

366 ISH and scRNA-seq analyses, as described below.

368 The most immature VZ stage, the neuroepithelium (VZ1), was represented by cells with 369 the highest expression of early mitotic markers such as Hes1 and Id4 (Kageyama et al., 370 2008) and roughly fit within cl-5 and cl-6 (Figures 5D, S7A). More mature VZ cells (VZ2;

371 perhaps radial glia), organized as a diagonal zone in the UMAP plot, were characterized

372 by expression of $W n t 7 b, I d 3$, and Ttyh1; they were roughly contained within $\mathrm{cl}-12$ and

373 the left part of $\mathrm{Cl}-1$ (Figures $5 \mathrm{E}, \mathrm{S7F}, \mathrm{G}$ ). This zone also had high expression of Hes5 
bioRxiv preprint doi: https://doi.org/10.1101/2021.01.11.426285; this version posted January 12, 2021. The copyright holder for this preprint (which was not certified by peer review) is the author/funder, who has granted bioRxiv a license to display the preprint in perpetuity. It is made available under aCC-BY-NC-ND 4.0 International license.
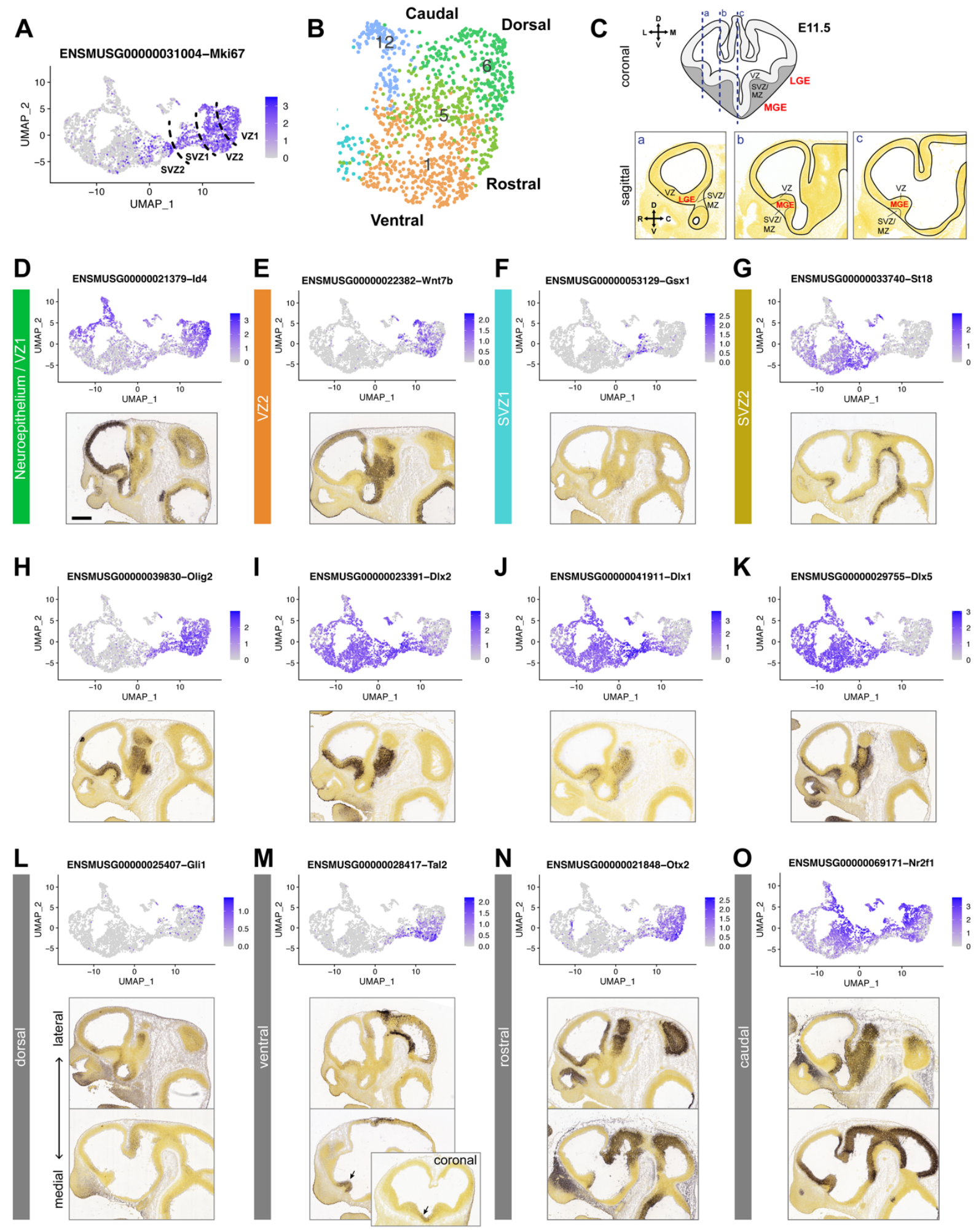
Figure 5: Separation of mitotic progenitors in E11.5 MGE.

(A) Visualization of single cells from E11.5 MGE by UMAP, colored by expression of the mitotic marker Mki67. Dotted lines show proposed boundaries for cells from VZ1, VZ2, SVZ1 and SVZ2. (B) Region of UMAP covering the VZ cells, colored by TF-defined cell cluster to show proposed regional identities (also shown in Figure 4E). (C) Top: coronal schematic showing the positions (blue dotted lines) of parasagittal sections used to illustrate regional gene expression patterns. Bottom: reference images of parasagittal sections from the ABA with the relevant subpallial anatomical domains labeled. (D-O) Gene expression UMAPs and representative ISH on E11.5 medial parasagittal sections from the ABA showing expression domains for markers of various mitotic cell identities. (D-G) Gene markers for VZ-SVZ developmental stages (Id4, Wnt7b, Gsx1, St18). Colored labels correspond to main cell cluster identity for those cells. (H-K) UMAPS and ISH for Olig2 and DIx2/1/5, genes that help define SVZ1 and SVZ2 identity. (L-O) Gene markers with known regional expression patterns (Gli1, Tal2, Otx2, Nr2f1) in the MGE VZ correlate with spatially defined UMAP locations, corresponding to the schematic in (C). Later and medial sections are shown. (M) Tal2 expression is highest at the medial level; coronal inset shows that this corresponds to the ventral MGE (arrow). Additional genes marking these developmental stages and regional identities are shown in Figures S7-8. Scale bar: $500 \mu \mathrm{m}$.

375 and Fabp7, which mark radial glia (Feng et al., 1994; Kageyama et al., 2008) (Figure

376 S7E,H). Other genes strongly marked both VZ zones (Eisenstat et al., 1999; Petryniak

377 et al., 2007; Roychoudhury et al., 2020), including Lhx2, Rest, and Rgcc (Figure S7B-

378 D). Genes like $A s c / 1, G s \times 2$ and $D / x 2$ began expression in the VZ as scattered cells both

379 in the UMAP plot and by ISH (Figures 5I; S7J,K).

381 SVZ organized into the progressively more mature SVZ1 in cl-9 and SVZ2 in cl-3. We

382 assigned SVZ1 identity to cl-9, based on overlapping Olig2 and D/x2 expression

383 (Petryniak et al., 2007) (Figure 5H,I). Gsx1 showed perhaps the most specific SVZ1

384 periventricular expression by ISH and was largely confined to cl-9 (Figure 5F). Cells in

385 this 'isthmus' cluster also expressed high levels of known SVZ genes (Asc/1, Gsx2 and

386 Hes6) (Long et al., 2009; Porteus et al., 1994; Roychoudhury et al., 2020) and

387 neurogenic transition markers (Btg2) (Haubensak et al., 2004) (Figure S7J-L).

388 Gadd45g, a marker of intermediate progenitors in the cortex (Yuzwa et al., 2017), was

389 also highest in $\mathrm{cl}-9$. SVZ2 identity was linked to $\mathrm{cl}-3$. This most mature progenitor state

390 was associated with the loss of Olig2 and high levels of D/x1,2, and 5 (Eisenstat et al., 
391 1999; Petryniak et al., 2007) (Figure 5H-K). By ISH, cl-3/SVZ2 markers (e.g. Prox1, Sp9

392 and St18) were expressed in a distinct layer of cells superficial to the SVZ1 markers

393 (Figures 5G, S7M,N). Insm1 and Is/1 appeared to be expressed in both SVZ1 and SVZ2

394 (Figure S7O,P). Cl-8 and cl-11 may represent the earliest stage of neuronal

395 commitment as SVZ2 cells exit the cell cycle; these clusters are discussed further

396 below.

397

398 Leveraging canonical correlation analysis of MGE cells across both $\mathrm{C} 1$ and 10x

399 datasets and ISH data, we found transcription factors and other markers that

400 distinguished the regionally distinct $h s 1538$ and $h s 1056$ populations (Figure S6).

401 Markers expressed in cells at the top of the UMAP plot (e.g. Nkx6-2, Gli1 and Gli2)

402 indicated dorsal MGE identity (Figures 5L, S8A,B). Dach2, a novel marker in this

403 category, occupied a similar location in the UMAP plot and was expressed in the dMGE

404 by ISH (Figure S8C). In the lower part of the UMAP plot, the genes expressed (Shh,

405 Slit2 and Tal2) indicated ventral (v)MGE identity (Hoch et al., 2015b) (Figures 5M, S8D-

406 E). The highest expression of VMGE genes was observed in medial sagittal planes of

407 ISH sections (Figure S8D-E). Bcan, Dach1 and Sulf1 may also mark vMGE progenitor

408 identity (Figure S8F-H). Molecular markers of MGE rostrocaudal position were also

409 identified in the UMAP plot. The rostral MGE had high Otx2 expression (Hoch et al.,

410 2015b) whereas the caudal MGE was marked by high Nr2f1 and Nr2f2 expression (Hu

411 et al., 2017) (Figures 5N,O, S8M). The preoptic area (POA) and pre-optic hypothalamus

$412(\mathrm{POH})$ are contiguous with the caudal mitotic zone in the MGE. Cl-12 may represent a

413 mixture of POA2 and POH progenitors, based on expression of Nkx6-2, Dbx1, and 
414 Pax6 (Flames et al., 2007) (Figures S8B,O,P). POA1 cells lack these markers and have

415 higher expression of Etv1 (Flames et al., 2007); these cells may be intermixed with

416 MGE cells within $\mathrm{cl}-1$. The septum is contiguous with the rostral MGE, and the septal

417 markers Fgf15 (Borello et al., 2008), Zic1 and Zic4 (Inoue et al., 2007; Rubin et al.,

418 2010) were also expressed by rostral MGE progenitors (Figure S8I,J). Pou3f1 and

419 Cntnap2 were novel markers of rostral cells (Figure S8K,L), whereas Ptx3 was a new

420 caudal MGE marker (Figure S8N). These patterning markers, including Id4, Otx2, and

421 Tcf7l2, also distinguished $h s 1538$ (rostrodorsal biased) and hs1056 (caudoventral

422 biased) cells (Table S4), validating the regional identity evident among MGE

423 progenitors. Thus, this enhancer labeling and TF-curated approach identified TF

424 expression gradients capturing early MGE regional patterning among progenitors.

426 Emergence of MGE neuronal lineages revealed by differential enhancer labeling

428 Postmitotic clusters were identified as the precursors of distinct MGE-derived cell

429 lineages: GABAergic interneurons destined for the cortex (CINs) or other structures,

430 GABAergic projection neurons, and cholinergic neurons (Figure 6A). Importantly, some

431 of these cell types appeared to emerge in different spatial subdomains of the MGE

432 based on marker gene expression. Early-born neurons emerge in SVZ2 and make up

433 the $M Z$, which can be further visualized via a topological projection map which

434 preserves spatial relationships across the telencephalon (Puelles et al., 2016;

435 Silberberg et al., 2016) (Figure 6B-C). The organization and cellular outputs of the basal

436 ganglia primordia identified herein using this topological map of E11.5 telencephalon 
A

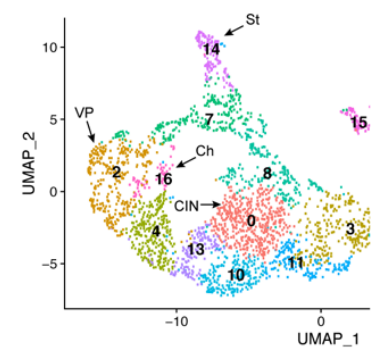

B

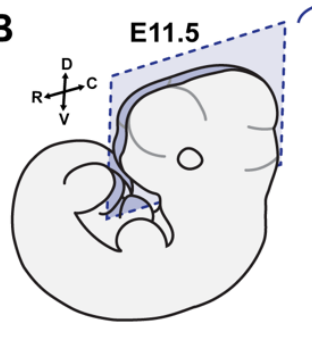

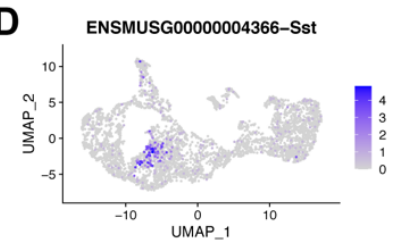

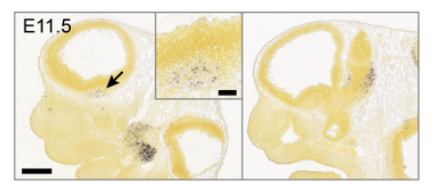

G
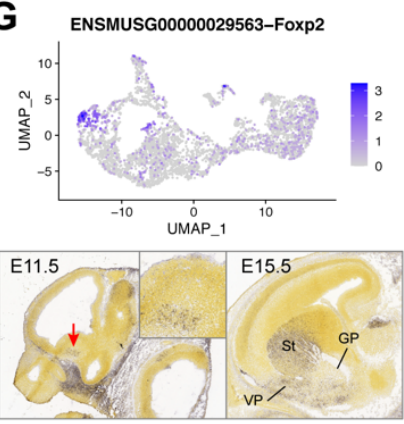

J
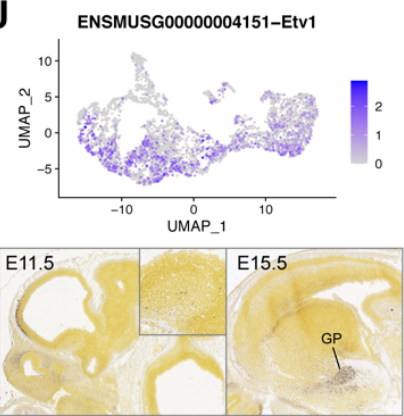

E
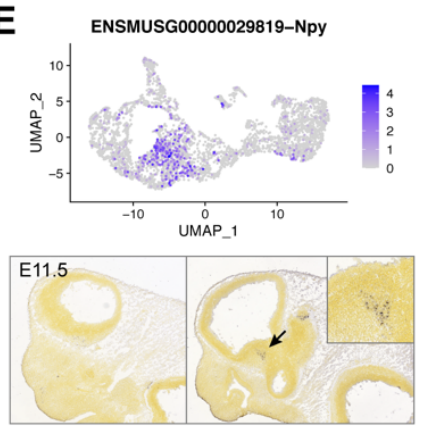

H
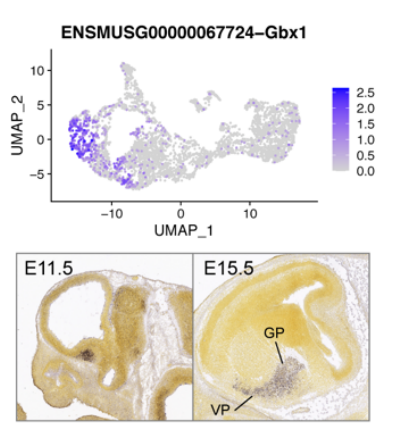

$\mathbf{K}$
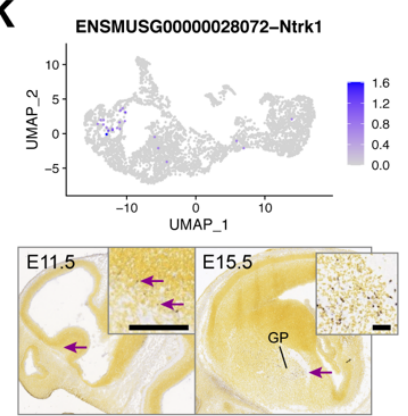

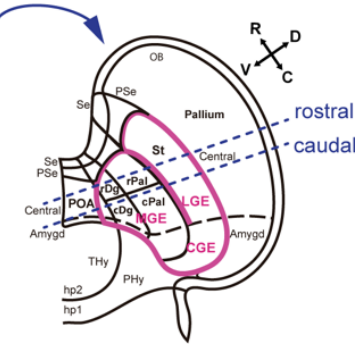

$\mathbf{F}$

C
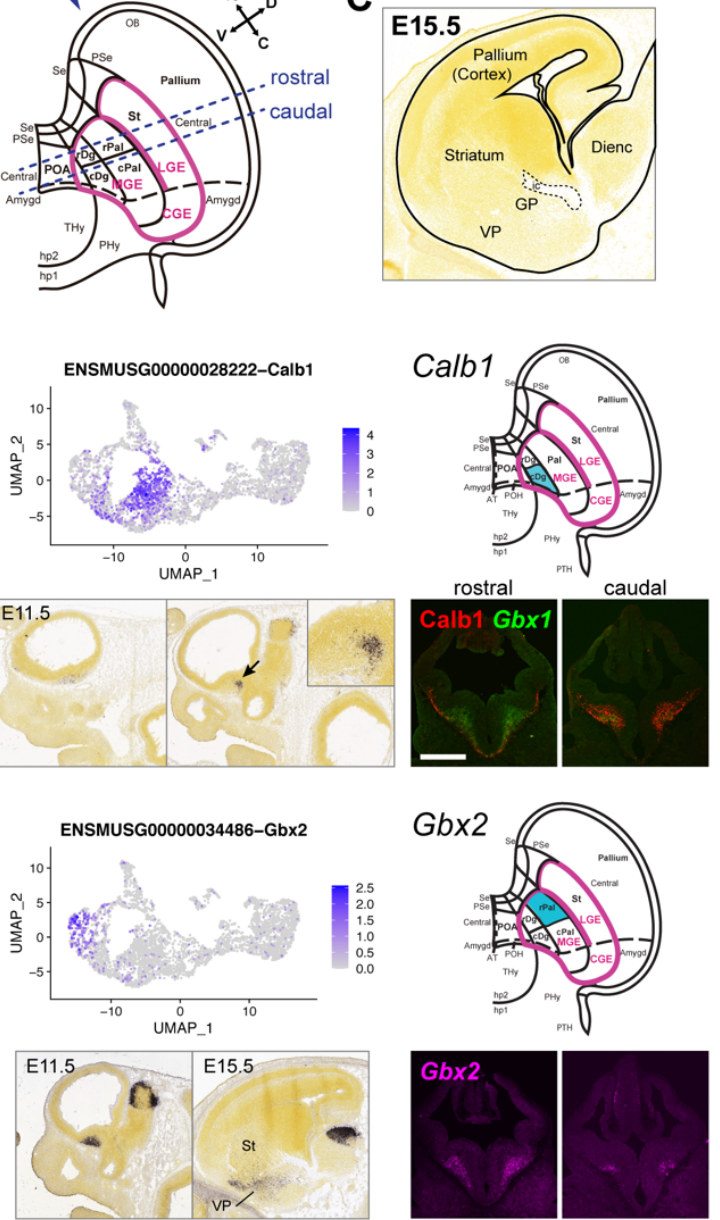

$\mathbf{L}$
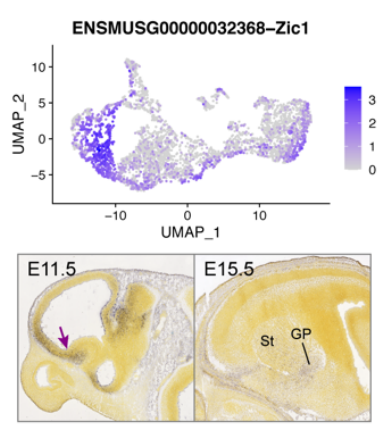

Gbx2
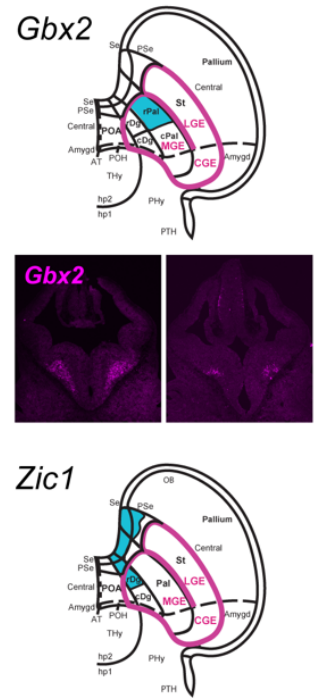

Zic1

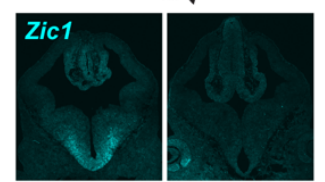

Figure 6: Separation of early neuronal lineages in E11.5 MGE.

(A) Portion of UMAP colored by TF-defined cell cluster (also shown in Figure 4E) covering postmitotic cells, with proposed identities of cell clusters labeled. Abbreviations: VP, ventral pallidum; CIN, cortical interneurons; St, striatal medium spiny neurons; GP, globus pallidus; Amygd, amygdala; Ch, cholinergic. (B) Collapsed 2D topological map of the E11.5 MZ, as viewed from the sagittal section plane indicated. The schema is a variant of that in Silberberg et al., 2016. Note the addition of rostral and caudal Pal (Pallidum) and Dg (Diagonal area). See Table S6 for abbreviations. Blue dotted lines (rostral and caudal) indicate positions of coronal fluorescent images shown in F, I and L. (C) Schematic depicting broad anatomical domains at E15.5. VP: ventral pallidum; GP: globus pallidus; Dienc: diencephalon. (D-L) Gene expression UMAPs and representative ISH on E11.5 parasagittal sections from the ABA showing expression of markers for distinct postmitotic neuronal lineages. Arrows indicate regions of higher 
438 correlate with progenitor zone anatomical map of (Flames et al., 2007) (Table S6).

439 Previous scRNA-seq studies have captured some of these emergent neuronal identities

440 but largely have not resolved the spatial organization underlying this process (Mayer et

441 al., 2018; Mi et al., 2018). Nkx2-1, Lhx6, and Lhx8 marked the MGE-derived postmitotic

442 neurons (Figure 4G), and these cell groups were further resolved in UMAP plots and

443 cluster assignments by their maturity state and presumed lineage. To analyze these

444 relationships, we focused on major emergent interneuron, GABAergic projection

445 neuron, and cholinergic lineages and the differential activity of $h s 192$ and $h s 799$ that

446 distinguishes these specific emerging neuronal populations.

448 Cluster $\mathrm{cl}-0$ and a subset of $\mathrm{cl}-13$ were composed primarily of $h s 799^{+}$and $h s 192^{-}$cells

449 and expressed immature interneuron markers. A subset of cl-8 included $h s 799^{+}$and

$450 h s 192^{+}$that expressed Mafb. Cl-0 showed the most consistent expression of immature

451 CIN markers including Calb1, Cux2, Erbb4, Lmo3, Maf, Npy, Sox6, Sst and Zeb2

452 (Figures 4G, 6D-F, S9A-G). Earlier lineage markers such as $L h x 8$ were reduced in

453 these cells. Subsets of cl-13 and cl-8 cells were Mafb+, but lack Maf and other CIN

454 markers. The small proportion of $\mathrm{Maf}^{+} h s 192^{+}$cells in cl-8 and cl-0 was consistent with

455 our C1 data and in line with fate mapping of a subset of $h s 192$-lineage cells to CINs

456 (Silberberg et al., 2016). Sentinel genes for cells from cl-0 and cl-13 were expressed in

457 a distinct area in the periventricular mantle zone of the caudal MGE based on ISH at

458 E11.5. We found that Calb1 expression is largely located in the caudal Diagonal (Dg) 
459 region, as illustrated on the topological map of the telencephalon (Figure 6F). The

460 caudal Dg also showed expression of Cux2, Erbb4, Npy, Sst, Maf, and Mafb; the latter

461 two genes are perhaps the most specific markers of MGE-derived CINs (Mckinsey et

462 al., 2013; Pai et al., 2019) (Figure 6D,E, S9A-D). Contiguous with this region, in more

463 lateral ISH sections, were cells expressing CIN markers, presumably migrating to the

464 LGE and/or CGE on their way to the cortex (e.g. Sst and Calb1 in Figure 6D,F).

465 Additionally, Adamts5, Bend4, Dlgap1, Kitl and Rai2 may be novel markers of immature

466 CINs (Figures S9H,I). Based on putative maturation states and overlapping

467 transcriptional signatures, immature interneurons from these three clusters can be

468 presumptively mapped back to late progenitor cells in SVZ2 (cl-3) via cl-11 for cl-0, cl-10

469 to $\mathrm{cl}-11$ for $\mathrm{cl}-13$, and within $\mathrm{cl}-8$. These findings map the neurogenic progression and

470 distinct spatial niches for emerging early MGE-derived interneurons, including $h s 799^{+}$

471 cells that contribute to $\mathrm{SST}^{+} \mathrm{CINs}$, as well as other populations.

472

473 Of particular interest, the scRNA-seq signatures and corresponding ISH patterns

474 suggested at least two distinct classes of GABAergic projection neurons differentially

475 labeled by $h s 799$ and $h s 192$ and distinguished by $G b x 1^{+}$or Zic1+ expression,

476 respectively, that originate in different parts of the MGE (Figure 6H,L). The first

477 GABAergic projection class was preferentially labeled by $h s 799$ and expressed Gbx1,

478 with more restricted expression of Foxp2 and Gbx2, and corresponded to Shh ${ }^{+} \mathrm{cl}-13$

479 cells and to subsets of cl-4 and cl-2 (Figures 6G-I, S8D). Based on a distribution on the

480 UMAP, Gbx1, Kitl, Lmo3, Sox6, Th, Tle4, Tshz2, Zeb2 and Zic1 (Flandin et al., 2010;

481 Mckinsey et al., 2013) are additional markers for cells from cl-2, cl-4, and cl-13 (Figures 
$4824 \mathrm{G}, 6 \mathrm{~L}, 7 \mathrm{D}, \mathrm{S} 9 \mathrm{E}-\mathrm{H}, \mathrm{J}, \mathrm{K})$. These cells were presumptively mapped to less mature states

483 in $\mathrm{cl}-10$ and $\mathrm{cl}-1$ (for $\mathrm{cl}-13$ only) or $\mathrm{cl}-4$ and $\mathrm{cl}-11$ (for $\mathrm{cl}-2$ ). On the topological map of the

484 MGE, Gbx2 expression was in the rostral portion of the pallidal (Pal) subdivision (Figure

485 6I). Anatomically, the E15.5 expression domains of Foxp2, Gbx2, Gbx1 and Etv1,

486 determined by ISH, were nested along the pallidum's radial axis. Foxp2 expression was

487 largely superficial, possibly in the ventral pallidum (Campbell et al., 2009) (Figure 6G).

488 Gbx1 expression encompassed the ventral pallidum and the entire globus pallidus

489 (Figure $6 \mathrm{H}$ ). Gbx2 included the ventral pallidum and part of the globus pallidus (Chen et

490 al., 2010) (Figure 6I). Etv1 was expressed throughout the globus pallidus but in few

491 cells in the ventral pallidum (Flandin et al., 2010) (Figure 6J). Foxp2 ${ }^{+}$cells become

492 superficial pallidal projection neurons (i.e. ventral pallidum), whereas other cl-2 cells,

493 and some cl-4 and cl-13 cells, contribute to deeper pallidal structures (i.e. globus

494 pallidus). Thus, we propose these populations that share increased $h s 799$ activity

495 branch from immature neuron types within $\mathrm{cl}-2, \mathrm{cl}-4$, and $\mathrm{cl}-13$.

497 The second GABAergic lineage expressed Zic1, as well as more restricted expression

498 of Zic3 and Zic4, and was enriched for cells with high hs192 activity (Figure 6L). Zic TFs

499 ISH MGE expression was restricted to the rostroventral MGE and overlapped little with

500 Gbx2; Zic1 may not overlap with the more caudal CIN markers (Figure 6D-F; S10A-D).

501 On the topological map, Zic1 expression was restricted to the rostral Dg region, and

502 was continuous with Zic1 expression in the septum (Figure 6L). As noted, Zic1 was also

503 expressed in the VZ and SVZ of the rostral MGE and septum, suggesting that this

504 progenitor zone generates the $\mathrm{Zic1}^{+}$postmitotic cells in $\mathrm{cl}-11$ and $\mathrm{cl}-4$. It is unclear from 
505 ISH how Zic1-associated GABAergic projection neurons are spatially organized within

506 the GP, though the spatial organization appears more diffuse across the GP. These

507 hs192-biased Zic1+ cells may constitute a distinct cell type within the GP.

508

509 Cells in cl-16 were exclusively enhancer $h s 192^{+}$and $h s 799^{-}$and expressed definitive

510 cholinergic marker genes. Among these, Ntrk1 (Sanchez-Ortiz et al., 2012) was the

511 most specific to this cluster (Figure 6K). Although Ntrk1 expression was weak in ISH at

512 E11.5, scattered positive cells were visible in the striatum and GP by E15.5, consistent

513 with the distribution of cholinergic interneurons (Figure 6K). Cl-16 cells also expressed

$514 G b x 1, G b \times 2$, Is/1 and Zic4, all of which are cholinergic lineage markers (Fragkouli et al.,

515 2009; Chen et al., 2010; Magno et al., 2017; Asbreuk et al., 2002; Elshatory and Gan,

516 2008), as well as Zic1, Zic2, and Zic5 (Figures 6H,I,L, S9L,M). Fgf15 appeared to be a

517 novel marker of this population, but its expression was not maintained as cells mature

518 (Figure S9N). A subset of $h s 192^{+} \mathrm{cl}-4$ cells also expressed some of these markers (e.g.

519 Zic2) and may represent a less mature state or another type of cholinergic neuron. Fgf8,

520 Fgf17, and Nkx2-1/Zic4 fate mapping provides evidence that these cells arise from the

521 junction of the rostromedioventral MGE with the septum (Magno et al., 2017; Hoch et

522 al., 2015a), consistent with the expression of Zic1.

523

524 The final postmitotic group, $\mathrm{cl}-7$, included two populations: the upper part was almost

525 exclusively $h s 192^{+}$, while the lower part also contained hs $799^{+}$cells. The upper part of

$526 \mathrm{Cl}-7$ was $N k \times 2-1^{-}$and strongly expressed Six3 and Sp8 (Figure S9O,P), suggesting a

527 CGE-derived CIN or LGE-derived olfactory bulb IN identity (Long et al., 2007). The 


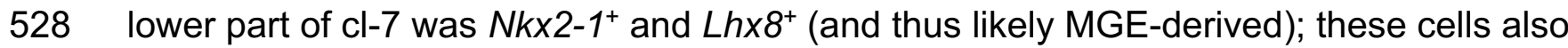

529 expressed genes shared by cl-2 and cl-14, including Id4 and Tle4 (Figures 5D, 7D).

530 Earlier states of cl-7 cells appeared to map back to cl-8 then SVZ2 cl-3 cells, with Nr2f2

531 expression in these clusters indicating caudal MGE origin (Figure S8M).

533 Across described postmitotic populations, enhancer-labeled scRNA-seq and ABA ISH

534 indicated specific spatial localizations for cell types with divergent transcriptomic

535 identities. To verify this finding, we performed fluorescent immunohistochemistry (IHC)

536 and ISH to map protein expression across coronal sections for four sentinel genes

537 representative of these populations (Figure 6F,I,L). We examined Calb1 for early CINs,

538 predicted to be located in caudal Dg; Gbx1 and Gbx2 for GABAergic projection neurons

539 in rostral Pal; and Zic1 for rostral Dg GABAergic projection and cholinergic populations.

540 These experiments validated that early born neuronal populations indeed exhibit spatial

541 segregation to specific rostrocaudal and dorsoventral zones of the MGE MZ. Thus, our

542 experiments captured novel lineage progression and associations between spatial and

543 transcriptomic identity of E11.5 MGE-derived neuron populations.

544

545 Validation of MGE enhancer-labeled progenitor and postmitotic populations

547 The combination of enhancer labeling and scRNA-seq paired with ISH defined distinct

548 populations marked by progenitor ( $h s 1538$ and $h$ s1056) and postmitotic ( $h s 192$ and

549 hs799) enhancers in the MGE. We used ISH and co-labeling to validate transcripts that 550 distinguish cells between these postmitotic and mitotic enhancer pairs. We found subtle 
551 but detectable transcriptomic differences between MGE progenitor cells labeled by

$552 h s 1538$ and $h s 1056$ corresponding to regional spatial segregation of $h s 1538$ to dMGE

$553 \mathrm{VZ}$ and $h s 1056$ to VMGE (Table S4). In contrast, there were broad transcriptional

554 differences between the spatially intermixed populations of postmitotic cells labeled by

$555 h s 192$ and $h s 799$ in the MGE (Tables S3,S4, Figures 3,5). Between $h s 1538$ and

$556 h s 1056$, among the most informative transcripts for differential enhancer labeling were

557 Id4, Tcf7/2, Zkscan1, Otx2, and Satb1 (Table S4). While the differential scRNA-seq

558 signatures were subtle, ISH verified highest expression of the hs1538-associated

559 marker Id 4 in the VZ of the dorsal and rostral MGE where $h s 1538$ is active (Figure 7A).

561 In postmitotic populations, hs192 activity, which was biased toward GABAergic

562 projection neurons in the striatum and cholinergic interneurons, was associated with

563 higher levels of the transcription factor Tle4 and several members of the Zic TF family

564 including Zic1, Zic3, and Zic4 (Table S4). Conversely, hs799 activity favored

565 populations of early CIN lineages expressing markers including Mafb and Sst, in

566 addition to its activity in GABAergic projection neurons. As hs192 and hs799 MZ

567 populations spatially intermingle, ISH alone was insufficient to verify specificity. Thus, to

568 validate differential expression of genes across $h s 192$ and $h s 799$ labeled cells, we

569 performed co-labeling of $\mathrm{GFP}^{+}$enhancer-labeled cells and Tle4 and Zic1, two

570 transcription factors that were enriched in $h s 192^{+}$cells (Figure 7B,C-F). As expected,

571 enhancer-positive cells showed overlapping distributions in the MGE, but $h s 192$ activity

572 and Zic1 expression were highest in the rostroventral MGE and paraseptal region

573 (Figure 7C,F). In addition, Tle4 protein was expressed in significantly more hs192 GFP ${ }^{+}$ 
A
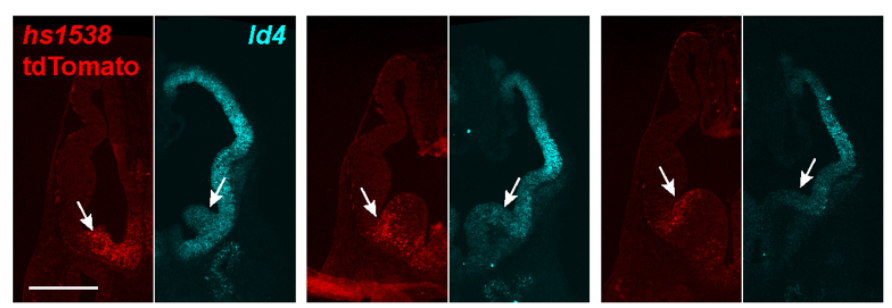

C

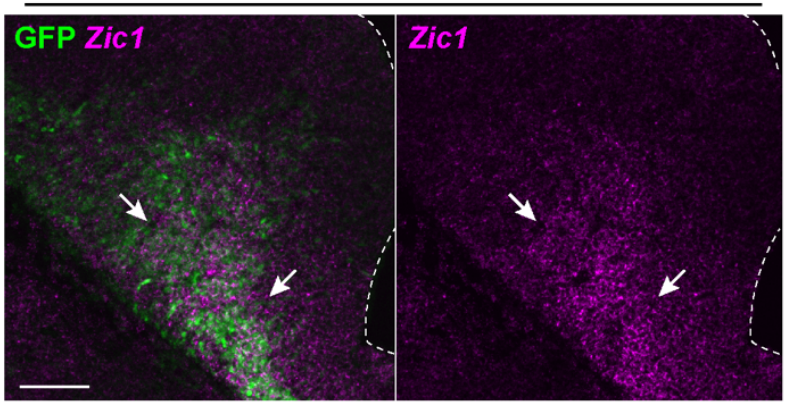

D

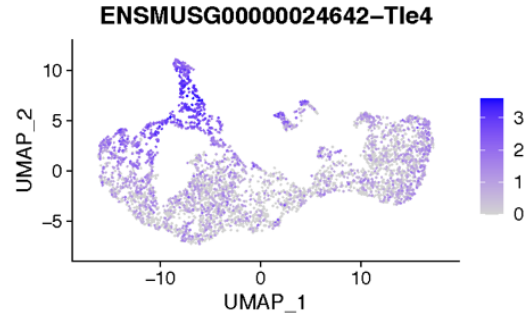

$\mathbf{F}$
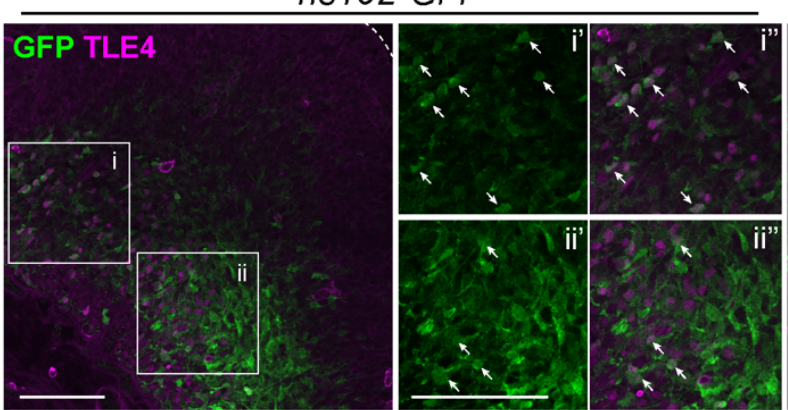

B

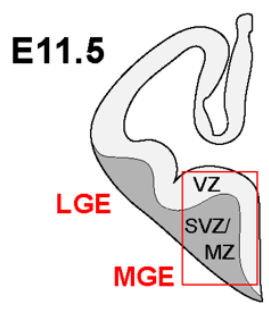

hs799-GFP

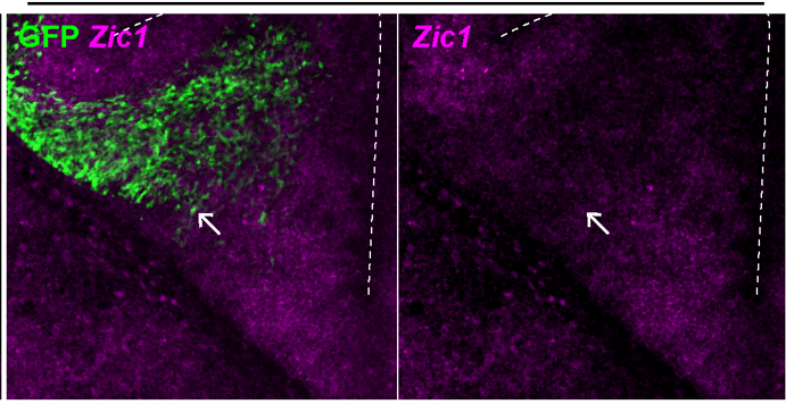

E

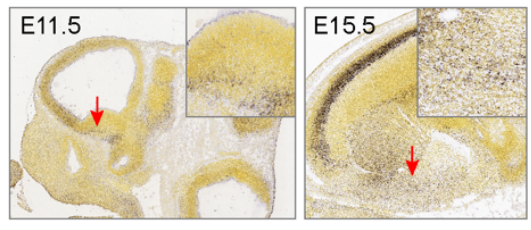

hs799-GFP
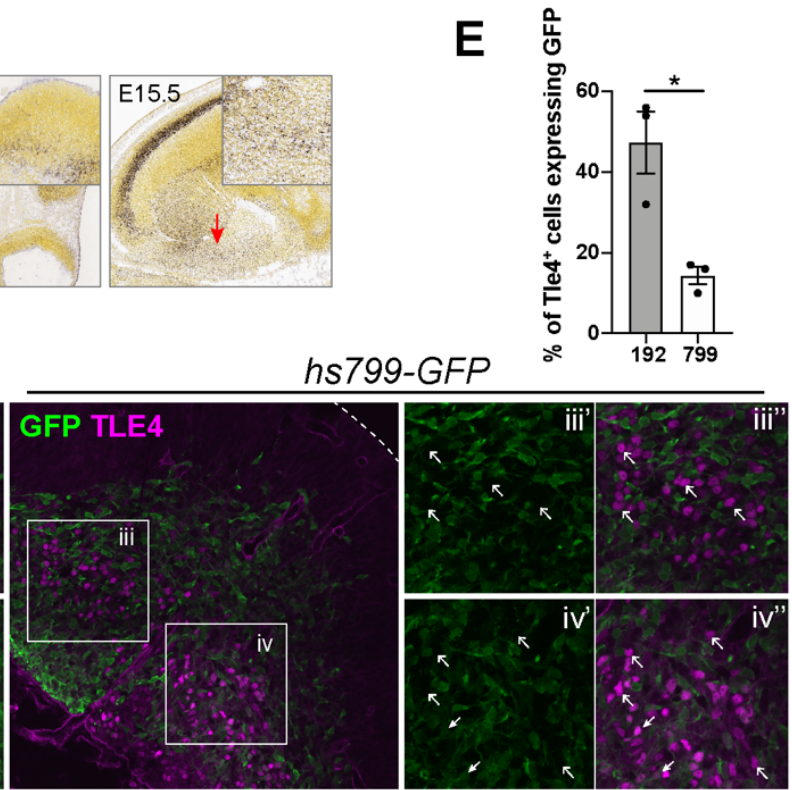

Figure 7: Spatial and epigenomic differences in E11.5 MGE enhancers.

(A) Endogenous tdTomato expression (red) in $h s 1538$ E11.5 coronal sections after tamoxifen administration at E10.75, and Id4 fluorescent ISH (cyan) on equivalent wildtype E11.5 sections. Arrows point to higher expression in the rostral and dorsal MGE VZ compared with ventral MGE. Scale bar: 500 $\mu \mathrm{m}$. (B) Schematic showing location (red box) of low magnification images shown in (C) and (F). (C) Zic1 fluorescent ISH (magenta) and GFP immunohistochemistry (green) on superimposed adjacent coronal sections of E11.5 hs192 and hs799 forebrain. Closed arrows indicate hs192 GFP overlaps with Zic1 expression in the ventral MGE. Open arrows indicate the ventral extent of $h s 799$ GFP cells, which does not extend into the region of high Zic1 expression. (D) UMAP of Tle4 expression and representative ISH on E11.5 sagittal sections from the ABA. (E) Quantification of percent of Tle4 cells that are also GFP positive from (F). (F) Immunohistochemistry for Tle4 and GFP on $h s 192$ and $h s 799$ coronal sections. Double-positive cells are indicated by closed arrows and GFP-negative cells are indicated by open arrows. Scale bars: $100 \mu \mathrm{m}$. Ventricular surface of MGE is indicated by dotted lines. 
575 cells compared to $h s 799 \mathrm{GFP}^{+}$cells (Figure 7E). Thus, ISH and co-labeling experiments

576 validate the results from scRNA-seq-based separation of enhancer-labeled MGE cells.

578 Discussion

580 Transcriptional profiling at single cell resolution has transformed our understanding of

581 the diversity of cell types in the brain. While initial efforts to catalogue brain cell types

582 have returned huge gains (Zeisel et al., 2018), new approaches are now needed to link

583 transcriptional identity to location, function, and developmental lineage. Here, we

584 combined novel enhancer-based cell labeling, TF-anchored clustering, and ISH-based

585 spatial annotation to map the neurogenic landscape of embryonic mouse basal ganglia.

586 Through this integrated approach, we illuminate enhancer activity in specific cells in vivo

587 and provide new insights regarding the specification paths for early GABAergic

588 neurogenesis in the ganglionic eminences.

590 Enhancer activity is often tightly restricted to specific cell types and developmental

591 stages (Dunham et al., 2012; Nord, 2013; Reddington et al., 2020), making enhancers

592 potent tools for genetic labeling and manipulation (Pattabiraman et al., 2014; Silberberg

593 et al., 2016; Visel et al., 2013). However, the specificity and pattern of enhancer action

594 in vivo at the single cell level has not been deeply explored. Most studies that examine

595 enhancer specificity in the brain have used image-based assays of reporter expression

596 (Visel et al., 2013) or orthogonal biochemical and epigenomic proxies to predict cell-

597 type specific enhancer activity (Dunham et al., 2012). In contrast, our approach 
598 represents a major advance in resolution for in vivo function-based modeling of

599 enhancer activity. Our results demonstrate that scRNA-seq can capture reporter

600 transcripts across histogenetic subtypes labeled by individual enhancers, thus

601 identifying enhancer-positive cells with high specificity even when enhancer activity was

602 low or limited to specific cell types. scRNA-seq analysis revealed the onset and offset of

603 enhancer activity as well as cell populations where each enhancer was active with fine-

604 scale resolution. Overall, this study reinforces the specificity of activity across individual

605 enhancers and increases resolution of enhancer activity mapping to offer a

606 representative perspective of in vivo single cell enhancer activity for seven evolutionarily

607 conserved neurodevelopmental enhancers.

608

609 Numerous genetic studies have probed mechanisms of BG development, with two

610 recent studies (Mayer et al., 2018; Mi et al., 2018) using scRNA-seq to follow BG

611 development and CIN lineage specification in E12.5-E14.5 embryonic mouse. Our

612 results capture an earlier time when alternative neuronal lineages originate, using

613 enhancer labeling, TF curation, and ISH to enable lineage tracking and spatial

614 resolution of progenitor and postmitotic populations across the GEs. Similar to other

615 studies, our results find transcriptomic differences among progenitor and postmitotic

616 cells across the GEs, with additional markers of maturation and postmitotic lineages

617 resolved via enhancer labeling. Further, integrating ISH data, we assigned likely

618 identities to four types of MGE progenitors; distinct MGE progenitor regions; and

619 enhancer-labeled subtypes of maturing MGE-derived interneurons, GABAergic 
620 projection neurons, and cholinergic neurons that together mature to form pallidal

621 structures or migrate to become interneurons in the cortex, amygdala, and striatum.

622

623 Progenitor cohorts with overlapping transcriptional states and region-specific signatures

624 were labeled by spatially distinct enhancers with VZ activity ( $h s 1538, h s 1056, h s 841$;

625 Figures 2,3,4). Similar to published studies at older ages (Mayer et al., 2018; Mi et al.,

626 2018), we found at E11.5 that scRNA-seq resolves the maturation gradient of BG

627 progenitor populations from NSCs in the VZ to postmitotic neuronal precursors in SVZ2

628 (Figure 5A). Comparison of $h s 1538^{+}$and $h s 1056^{+}$enabled the discovery of genes

629 whose expression in MGE progenitors defined rostrocaudal and dorsoventral axes. The

630 rostrodorsal $h s 1538^{+}$progenitors were enriched for Id4, Otx2, Tcf7l2, and Zic1 (Figures

$6313,5,7$; Table S4). In contrast, caudoventral progenitors were enriched for $N r 2 f 1 / 2$ and

632 Tal2 (Figure 5). Interestingly, Id4 is both associated with neuroepithelial progenitors

633 (Bedford et al., 2005; Yun et al., 2004) and biased toward rostrodorsal MGE VZ,

634 suggesting potential differences in progenitor state composition across regional

635 domains within E11.5 MGE VZ. Of note, progenitors segregated on the UMAP across

636 these axes when using a TF-anchored transcriptome analysis (Figure 5). We suggest

637 that positional information in E11.5 MGE progenitors is largely encoded by gradients of

638 these and other TFs rather than the expression of specific TF domains. Combinations of

639 TFs then activate region-specific enhancers such as $h s 1538$, as has been shown in

640 classic model of ectoderm patterning in drosophila embryos (Levine, 2008) and in the

641 cortical VZ (Pattabiraman et al., 2014). 
643 Early-born CGE, LGE and MGE GABAergic projection, cholinergic, and inhibitory

644 neuronal lineages are labeled by differential enhancer activities ( $h s 953, h s 599, h s 192$,

645 hs799, respectively) (Figures 3,4,6). Compared to the distinct MGE signature, LGE and

646 CGE cells had more similar transcriptomic identities, but diverged between $h s 599$ and

647 hs953 cells in later post-mitotic neuron clusters. Focusing on MGE, differential scRNA-

648 seq comparison of enhancers $h s 192$ and $h s 799$ in combination with ISH annotation

649 defined three spatially distinct regions in the MGE MZ that give rise to molecularly

650 distinct cells. CINs (expressing multiple markers) were detected in the caudal Diagonal

651 (Dg) region (Figure 6F). Gbx1/2+ cells were mapped to the Pallidal (Pal) region and

652 Zic $^{+}$cells to the rostral Dg regions (Figure $6 \mathrm{I}, \mathrm{L}$ ). We are intrigued by the possibility that

$653 \mathrm{Gbx}^{+}$and $\mathrm{Zic}^{+}$cells contribute to distinct types of MGE GABAergic neurons, including

654 within the GP, in addition to the cholinergic neurons already described (Chen et al.,

655 2010; Magno et al., 2017). Previous studies have not identified a specific MGE region

656 giving rise to CINs, nor spatially distinct zones for the generation of pallidal neurons and

657 CINs. This contrast to our findings may be because our analysis focused on a younger

658 age (E11.5) than most studies. Consistent with our results, (Puelles et al., 2016)

659 previously showed that Sst expression begins in the Dg region. It is probable that as

660 development proceeds, additional MGE regions also generate CINs, as suggested by

661 many studies (Mayer et al., 2018; Mi et al., 2018; Silberberg et al., 2016). Overall, the

662 results from these experiments define emerging GABAergic neuron types and elucidate

663 relationships between spatial and transcriptomic characteristics of lineages in

664 embryonic BG and in early mammalian brain development. 
666 Beyond BG neurogenesis, our study has broader implications for the application of

667 scRNA-seq to developing tissues. We demonstrate that anchoring scRNA-seq analysis

668 on TF transcripts reduces the weight of cell cycle-driven and technical sources of

669 variation (noise), improving the power of histogenetic cell type classification. Limiting

670 analysis to TF transcripts greatly improved resolution of regional identity and maturation

671 state for progenitors in the VZ and SVZ, consistent with the vast body of literature

672 defining TF gradients as the master regulators of lineage specification in the BG and

673 elsewhere. The extensive use of ISH annotations to define regional gradients enabled

674 us to identify and interpret patterns of cell identity captured in scRNA-seq data. Finally,

675 enhancer labeling enabled us to enrich, identify, and compare specific progenitor

676 populations and early neuronal lineages, capturing novel transcriptional signatures for

677 labeled lineages. Our results highlight the value and need for curated approaches in

678 scRNA-seq analysis (e.g. our focus on TF transcripts) and the utility of leveraging

679 orthogonal and accessory data, in this case ISH and enhancer labeling, to understand

680 complex developmental processes. This study thus represents a new frontier, pairing

681 scRNA-seq with functional analysis of enhancer activity in vivo and highlighting the

682 utility of combining enhancer-mediated expression for labeling and characterization of

683 specific cell types and offers insight into GABAergic neurogenesis in the embryonic

684 mouse BG. 


\section{ACKNOWLEDGEMENTS}

686 Next-generation sequencing was performed at the Center for Advanced Technology at

687 UC San Francisco and the UC Davis DNA cores. Confocal microscopy was performed

688 at the UC San Francisco Nikon Imaging Center, supported by NIH S10 Shared

689 Instrumentation grant 1S10OD017993-01A1. Cell sorting was performed at the UC San

690 Francisco HDFCC Laboratory for Cell Analysis, using instrumentation supported by NIH

691 grant P30CA082103. Additional cell sorting and C1 scRNA-seq were performed at the

692 UCSF Parnassus Flow Core (RRID:SCR_018206) supported in part by Grant NIH P30

693 DK063720 and by the NIH S10 Instrumentation Grant S10 1S10OD021822-01. 10x

694 scRNA-seq was performed by the UCSF IHG Genomics Core. L.S.-F. was supported by

695 the UC Davis Floyd and Mary Schwall Fellowship in Medical Research, the UC Davis

696 Emmy Werner and Stanley Jacobsen Fellowship, and by grant number T32-GM008799

697 from NIGMS-NIH. R.C.-P. was supported by a Science Without Borders Fellowship from

698 CNPq (Brazil). This work was supported by the following research grants. J.LR.R.:

699 NIMH R01 MH081880 and NIMH R37/R01 MH049428. A.S.N.: NIH/NIGMS R35

700 GM119831. Z.J.G.: Department of Defense Breast Cancer Research Program

701 (W81XWH-10-1-1023 and W81XWH-13-1-0221), NIH (U01CA199315 and DP2

702 HD080351-01), NSF (MCB-1330864), and the UCSF Center for Cellular Construction

703 (DBI-1548297), an NSF Science and Technology Center. Z.J.G. is a Chan-Zuckerberg

704 BioHub Investigator. L.P. was supported by Seneca Foundation (5672 Fundación

705 Séneca, Autonomous Community of Murcia) Excellency Research contract:

706 19904/GERM/15; project name: Genoarchitectonic Brain Development and Applications

707 to Neurodegenerative Diseases and Cancer. 


\section{AUTHOR CONTRIBUTIONS}

710 L.S.-F. and A.N.R. are listed as joint first authors, as each led components of the

711 experiments and analysis. J.L.R.R. and A.S.N. are listed as joint senior and

712 corresponding authors. L.S.-F., A.N.R., S.N.S., J.L.R.R., and A.S.N. designed the

713 experiments. Dissections, single-cell preparations and histology: A.N.R. and S.N.S.;

714 scRNA-seq library preparation: L.S.-F., A.N.R., and I.Z.; bioinformatics: L.S.-F., R.C.P.,

715 K.J.L., C.S.M., T.E.R., Jr., and A.S.N.; in situ hybridization prioritization and scoring:

716 S.N.S., G.L.M., M.H., C.T., and H.Z. Topological map: L.P. and J.L.R.R. L.S.F., A.N.R.,

717 J.L.R.R., and A.S.N. drafted the manuscript. All authors contributed to manuscript

718 revisions.

720 DECLARATION OF INTERESTS

721 J.L.R.R. is cofounder, stockholder, and currently on the scientific board of Neurona, a

722 company studying the potential therapeutic use of interneuron transplantation.

723 


\section{Methods}

\section{RESOURCE AVAILABILITY}

727 Lead Contact

728 Further information and requests for resources and reagents should be directed to and

729 will be fulfilled by the Lead Contacts, Alex S. Nord (asnord@ucdavis.edu) and John L.

730 R. Rubenstein (john.rubenstein@ucsf.edu).

Materials Availability

733 The enhancer transgenic mouse lines used in this study have been previously published

734 (Silberberg et al., 2016) and deposited to the MMRRC repository.

736 Data and Code Availability

737 The datasets generated during this study are available on GEO (accession TBD). The

738 analysis codes used for this study can be found on the Nord Lab Git Repository

739 (https://github.com/NordNeurogenomicsLab/).

\section{EXPERIMENTAL MODEL AND SUBJECT DETAILS}

\section{Mice}

743 The enhancer transgenic mouse lines used in this study have been previously published

744 (Silberberg et al., 2016) and deposited to the MMRRC repository. All animal care,

745 procedures, and experiments were conducted in accordance with the NIH guidelines

746 and approved by the University of California, San Francisco animal care committee's

747 regulations (Protocol AN180174-02). Pregnant dams were housed in mating pairs, or 
748 singly housed with additional environmental enrichment. Mice were housed in a

749 temperature-controlled environment $\left(22-24^{\circ} \mathrm{C}\right)$, had ad libitum access to food and water,

750 and were reared in normal lighting conditions (12-h light-dark cycle). Embryos of either

751 sex were used, and all embryos of the correct genotype from a single litter were pooled

752 as a single biological replicate for all sequencing experiments.

753

754 We used mice from 7 previously published enhancer transgenic lines (Silberberg et al.,

755 2016): hs192-CreER ${ }^{T 2}$-IRES-GFP, hs599-CreER ${ }^{T 2}-I R E S-G F P$, hs799-CreER ${ }^{T 2}-I R E S-$

756 GFP, hs841-CreER ${ }^{T 2}$-IRES-GFP, hs953-CreER ${ }^{T 2}$-IRES-GFP, hs1056-CreER ${ }^{T 2}$-IRES-

757 GFP and $h s 1538-C r e E R^{T 2}$-IRES-GFP, herein referred to simply by their enhancer ID,

758 e.g., hs192. Enhancer line hemizygous transgenic male mice were mated to CD-1

759 wildtype or Ai14 tdTomato Cre-reporter female mice (MGI ID: 3809524) (Madisen et al.,

7602010 ) to obtain embryos for experiments. All transgenic mice were maintained on a

761 mixed background outcrossed to CD-1. For inducible tdTomato labeling of enhancer

762 positive cells with the $h s 1538$ line, Ai14 reporter female mice were mated to enhancer

763 transgenic males and dosed with $55 \mathrm{mg} / \mathrm{kg}$ tamoxifen dissolved in corn oil at $6 \mathrm{pm}$ the

764 day before embryo harvest (E10.75 for harvest at E11.5). $14 \mathrm{mg} / \mathrm{kg}$ progesterone was

765 included to improve embryo survival.

766

767 METHODS DETAILS

768 C1 scRNA-seq

769 Cell isolation. 
770 Pregnant dams were sacrificed by $\mathrm{CO}_{2}$ inhalation, confirmed by cervical dislocation.

771 E11.5 embryos were removed and placed into ice-cold Earle's Balanced saline solution

772 (EBSS). Transgene-positive embryos were identified by screening on a fluorescent

773 microscope. In a clean dish with ice-cold EBSS, the MGEs, LGEs or CGEs were

774 dissected out and placed into a $1.5 \mathrm{~mL}$ Eppendorf tube containing EBSS on ice. Tissue

775 was pooled from all transgene-positive embryos in a single litter.

776

777 The tissue was dissociated in $300 \mu \mathrm{L}$ of $0.25 \%$ trypsin-EDTA solution supplemented

778 with $10 \mathrm{U} / \mathrm{mL}$ recombinant DNase I (Roche) for 15 minutes at $37^{\circ} \mathrm{C}$. Trypsinization was

779 stopped by addition of $300 \mu \mathrm{L}$ DMEM with $10 \% \mathrm{FBS}$, and the tissue was gently

780 triturated 10-15 times with a P1000 pipette and filtered through a $40 \mu \mathrm{m}$ filter to achieve

781 a single-cell suspension. The cells were spun down for 3 minutes at $500 \mathrm{rcf}$ and

782 resuspended in FACS buffer (EBSS + 0.5\% BSA + $2 \mathrm{mM}$ EDTA). DAPI (50 ng/mL final

783 concentration) was included to stain dead cells.

784

785 For gated samples, GFP- and tdTomato-positive cells were isolated by fluorescence

786 activated cell sorting on a FACSAria II flow cytometer (BD Biosciences). FACS gating

787 was set using a transgene-negative sample, and DAPI-positive dead cells were

788 excluded. Single-cell sorting mode was used to maximize sample purity. For ungated

789 samples, DAPI-stained cells were excluded but GFP-positive and GFP-negative cells

790 were collected.

791

$792 \quad$ C1 cell capture and cDNA generation. 
793 For unsorted and ungated experiments, cells were counted on a haemocytometer and

794 diluted to $150-300$ cells/ $\mu \mathrm{L}$ in FACS buffer. The cell mix was prepared and loaded onto

795 a Fluidigm integrated fluidics chip ( C1 $^{\text {TM }}$ Single-Cell mRNA Seq IFC, 5-10 $\mu \mathrm{m}$, \#100-

796 5759) on the Fluidigm C1 system according to the manufacturer's instructions.

798 For GFP- and tdTomato-gated samples, cells were sorted directly into the loading well

799 of the IFC (integrated fluidic circuit) according to the manufacturer's note

800 (https://www.fluidigm.com/articles/cell-sorting-directly-to-the-c1-ifc). Briefly, the IFC

801 plate was placed on the plate chiller of the FACSAria sorter, the inlets were covered

802 with PCR-plate sealing film, and the sort stream was directed to the cell loading well.

803 The film over the cell loading well was removed and $3 \mu \mathrm{L}$ of $\mathrm{C} 1$ cell suspension buffer

804 was added to the well. 1,500 cells were then sorted directly into the well. Finally, $1.5 \mu \mathrm{L}$

805 of FACS buffer was added to give a final volume of $7.5 \mu \mathrm{l}$ and a concentration of 200

806 cells $/ \mu \mathrm{L}$. The cell suspension was pipetted gently 2-3 times to mix, and cells were

807 loaded onto the IFC using the C1 machine according to the manufacturer's instructions.

808 After capture, each well of the IFC was visually examined on a Keyence microscope

809 and scored for the number of cells and presence or absence of cell debris.

811 Cell lysis, reverse transcription and cDNA amplification were performed on the Fluidigm

812 C1 machine according to the manufacturer's mRNA-seq protocol using the SMARTer

813 Ultra Low RNA Kit for the Fluidigm C1 System (Takara Bio \#634833). cDNA amplicons

$814(\sim 3 \mu \mathrm{L})$ were harvested into a 96-well plate containing $10 \mu \mathrm{L} C 1$ DNA dilution buffer per

815 well, as described in the protocol, and stored at $-80^{\circ} \mathrm{C}$ for library preparation. 
817 C1 scRNA-seq library preparation.

$8181 \mathrm{uL}$ of diluted cDNA per well was used for library preparation using the Nextera XT

819 DNA Sample Prep Kit (Illumina, \#FC-131-1096). Each C1 IFC was pooled into one

820 library, for a total of up to 96 samples per library. Sequencing library quality was

821 assessed using the high-sensitivity dsDNA assay in an Agilent Bioanalyzer.

822

823 Multiplexed 10x scRNA-seq library generation (MULTI-seq)

824 E11.5 MGE tissue was dissected as described above and dissociated using the Papain

825 Dissociation System (Worthington) with a modified protocol. MGEs pooled from a single

826 litter were incubated with $200 \mu \mathrm{L}$ papain solution supplemented with $10 \mathrm{U} / \mathrm{mL}$

827 recombinant DNase I (Roche) for 10 minutes at $37^{\circ} \mathrm{C}$ on a rocking platform, then spun

828 down for 3 minutes at 300 rcf. The papain solution was replaced with $200 \mu \mathrm{L}$ ice-cold

829 EBSS, and the tissue was gently triturated $~ 10$ times with a P1000 pipette to achieve a

830 single-cell suspension. The cells were spun down and resuspended in $200 \mu L$ EBSS for

831 fluorescence activated cell sorting on a FACSAria II machine (BD Biosciences). An

832 aliquot of cells was stained with trypan blue and counted on a haemocytometer. The cell

833 suspension was diluted if necessary with EBSS, aiming for $\leq 500,000$ cells in a volume

834 of $200 \mu \mathrm{L}$.

835

836 Dissociated cells were labeled with barcoded lipid-modified oligonucleotides (LMOs) as

837 previously published (McGinnis et al., 2019), using a different barcode for each single-

838 litter pooled MGE sample. Excess LMOs and papain were quenched by adding $1 \mathrm{~mL}$ of 
839 ovomucoid/BSA inhibitor (Worthington). Barcoded cells were spun down for 5 minutes

840 at $500 \mathrm{rcf}$ and resuspended in $300 \mu \mathrm{L}$ EBSS with $1 \% \mathrm{BSA}$. DAPI $(50 \mathrm{ng} / \mathrm{mL}$ final

841 concentration) was included to stain dead cells. Just prior to sorting, cells were passed

842 through a $40 \mu \mathrm{m}$ filter to remove any remaining clumps.

843

844 GFP-positive cells were isolated by FACS as described above. Cells were sorted into

845 Lo-Bind Eppendorf tubes containing EBSS with 1\% BSA. Sorted, barcoded cell samples

846 were then pooled, spun down, and resuspended in a small volume of EBSS with $1 \%$

847 BSA ready for processing on the Chromium 10x system.

849 Single-cell cDNA libraries were generated using the Chromium Single Cell 3' GEM,

850 Library \& Gel Bead Kit (v3, PN-1000075) according to the manufacturer's instructions.

851 After the first cDNA clean-up step with 0.6x SPRI beads, the supernatant containing the

852 barcode library fraction was saved and processed as described previously (McGinnis et

853 al., 2019). The barcode library (5\%) and cDNA library (95\%) were pooled for

854 sequencing on an Illumina NovaSeq SP lane.

855

856 C1 sequencing, alignment, and gene expression quantification

857 Libraries for the C1 scRNA-seq samples were sequenced on an Illumina HiSeq 4000

858 instrument using a single-end 50-bp protocol. Reads were uniquely aligned to the

859 mouse genome (GRCm38, modified to append a custom chromosome containing the

860 individual sequences of the transgenes CreER ${ }^{T 2}$, IRES, EGFP, and tdTomato) using

861 STAR (v2.7.0e) (Dobin, 2013), and read duplicates were removed using the Picard tools 
862 function MarkDuplicates (v2.18.4) (Picard Toolkit, 2019). Gene counts were generated

863 using subread featureCounts (v1.6.3) (Liao et al., 2014), to ENSEMBL GRCm38

864 release 95, using a customized gtf annotation file containing annotations for the four

865 transgenes. Gene counts were normalized to gene length and library size using reads

866 per kilobase of transcript, per million mapped reads (RPKM) or to counts per million

867 mapped reads (CPM) for downstream analysis and visualization.

869 10x sequencing, alignment, and gene expression quantification

870 Libraries for 10x MULTI-seq samples were sequenced on an Illumina NovaSeq SP

871 instrument. The raw 10x data was processed using CellRanger (v3.0.2) (Zheng et al.,

872 2017) using the custom mouse genome described above. Samples were demultiplexed

873 using the MULTI-seq (McGinnis et al., 2019) pipeline (https://github.com/chris-mcginnis-

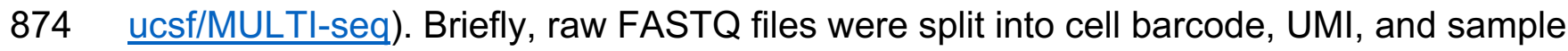

875 barcode sequences, then reads were parsed to generate a sample barcode UMI count

876 matrix. The cell barcode matrix was put through the MULTI-seq classification workflow

877 to identify single cells from unclassified and doublet/multiplet cells. A second round of

878 semi-supervised classification was performed to reclassify negative cells for potential

879 false negatives. The cell by gene UMI count matrix was loaded into Seurat (v3.2.2)

880 (Butler et al., 2018; Stuart et al., 2019) as a Seurat object, and normalized using the

881 Seurat function SCTransform (Hafemeister and Satija, 2019) with default parameters for

882 both full transcriptome and TF-curated analyses.

883

\section{C1 quality control}


885 Raw read quality was assessed using FastQC (v0.11.7) (Andrews, 2010). Aligned

886 library quality was assessed using RSeQC (v2.6.4) (Wang et al., 2012) for 5' or 3' bias,

887 exonic read distribution and GC content distribution. We also assessed quality of

888 individual samples by comparing the following distributions: total uniquely aligned reads,

889 total assigned reads, total expressed genes, percent mitochondrial reads, percent

890 ribosomal genes, and percent pseudogenes. Samples with greater or less than 2

891 standard deviations from the mean in any of the above metrics were discarded. In

892 addition, samples scored as 'two or more cells' during visual inspection were removed

893 from analysis. Pseudogenes, ribosomal genes (ribosomal subunit and rRNAs),

894 mitochondrial genes, and six sex-linked genes (Xist, Eif2s3x, Kdm6a, Ddx3y, Eif2s3y,

$895 K d m 5 d$ ) (Armoskus et al., 2014) were removed from the data matrix.

896

897 We used the R package Seurat (v3.2.2) (Butler et al., 2018; Stuart et al., 2019) for

898 feature selection, clustering and visualization. We compared two normalization

899 approaches for quality control and downstream analysis. RPKM-normalized data was

900 used to create two Seurat objects, with minimum.cells set to 10 , and minimum.genes

901 set to 2,000 . We applied a manual $\log 1 \mathrm{p}$ transformation to the RPKM data to generate

902 normalized data in the 'data' slot of the Seurat object. We applied regression for FACS-

903 processing state and total mapped reads on the gene count data using ScaleData. To

904 define proliferative state and cell cycle phase, we used the Seurat function

905 CellCycleScoring on a published list of cell cycle genes (Kowalczyk et al., 2015) and

906 calculated G1/S and G2/M phase scores for each cell. We then calculated the 
907 difference between $\mathrm{G} 1 / \mathrm{S}$ and G2/M to define whether a cell was mitotic (M) or post-

908 mitotic (PM).

909

910 We also compared our results to those generated with raw count data normalized using

911 the NormalizeData function with scale.factor set to $1 \mathrm{e} 6$ to generate CPM-normalized

912 data. Results were largely similar between RPKM- and CPM-normalized data and only

913 the RPKM-normalized results are reported. We note that short genes were more likely

914 to be considered highly variable using CPM-normalized data (data not shown).

915

916 10x quality control

917 We used the MULTI-seq pipeline described above to remove negative or multiplet cells

918 from downstream analysis. After subsetting data to only singlets, we used the full

919 transcriptome data to perform cell clustering with Seurat, which split cells into two

920 populations based on mitochondria RNA (mtRNA) expression: one population with high

921 mtRNA expression ( $>5 \%$ total UMIs) and one with low expression ( $<5 \%$ total UMIs). We

922 removed all cells from the high mtRNA expression population, and repeated cell

923 clustering using the cleaned cells. We identified one outlier cluster likely corresponding

924 to hematopoietic cell lineages based on expression of hemoglobin genes, and removed

925 that cluster, resulting in 4,001 cells for downstream analysis.

926

927 C1 transcription factor (TF)-based clustering

928 From the full dataset, we extracted counts for 689 transcription factors with mRNA in

929 situ hybridization data available in the Allen Developing Mouse Brain Atlas (Lein et al., 
930 2007) that were scored for expression in E11.5 and E13.5 basal ganglia and cortex

931 (Table S2). Of the 689 scored transcription factors, 455 were expressed in $>10$ cells in

932 our dataset. These 455 genes were used for dimensionality reduction using principal

933 components analysis (PCA) and Uniform Manifold Approximation and Projection

934 (UMAP) (Mclnnes et al., 2018) as visualization tools. Following PCA, we used jackstraw

935 analysis with 100 iterations to define statistically significant $(p$-value $<0.05)$ principal

936 components (PCs) driving variation. The first 13 significant PCs were used to define

937 clusters using FindNeighbors (using k. param = 5, nn.method = "annoy", annoy $\cdot$ metric $=$

938 "euclidean") and FindClusters (using resolution = 1.2, algorithm = 2, group.singletons =

939 F). To generate the UMAP visualization we used Seurat's RunUMAP (reduction = "pca",

940 n.neighbors $=15$, n.epochs $=1000$, negative. sample.rate $=10)$. Using this approach,

941 we defined 12 clusters.

942

\section{$94310 x$ transcription factor (TF)-based clustering}

944 We used a similar approach as described above for the C1 clustering. Of the 689

945 scored transcription factors, 463 were expressed in greater than 10 cells. These 463

946 genes were used for downstream processing. The first 14 significant PCs were used to

947 define clusters using FindNeighbors (using k. param = 15, nn. method = "rann",

948 annoy.metric = "euclidean") and FindClusters (using resolution $=1.4$, algorithm $=2$,

949 group.singletons $=$ F). To generate the UMAP visualization we used Seurat's RunUMAP

950 (reduction $=$ "pca", n.neighbors $=20$, n.epochs $=500$, negative $\cdot$ sample $\cdot$ rate $=15$ ). Using

951 this approach, we defined 18 clusters. 


\section{Differential expression analysis}

954 For differential expression (DE) analysis across TF-based clusters in both $C 1$ and 10x

955 datasets, we expanded to all expressed genes and used Seurat's FindAIIMarkers.

956 Briefly, one cluster of cells was compared to all other cells using a Wilcoxon rank-sum

957 test. We only considered genes that were expressed in at least $25 \%$ of cells in either

958 population and had $\log _{\mathrm{e}}$-fold-change greater than 0.25 between populations. Genes with

959 adjusted $p$-values $<0.05$ were considered statistically significant. We also performed

960 DE analysis between enhancer groups (e.g. MGE-hs192 vs MGE-hs799, or MGE-

961 hs192-PM vs MGE-hs799-PM) using the same parameters.

962

\section{Diffusion mapping pseudotime analysis}

964 We performed diffusion mapping via the R package destiny (Angerer et al., 2016) on the

965 TF-based C1 dataset. Briefly, the log-normalized TF-curated RPKM data was passed to

966 the destiny function $\mathrm{dm}$, which generates 50 diffusion components and corresponding

967 cell eigenvalues. We assessed differential expression along the diffusion components

968 using a generalized additive model via the gam function in $\mathrm{R}$.

\section{Random forest classification}

971 We used random forest (Breiman, 2001) via the R package randomForest on the TF-

972 based $\mathrm{C} 1$ dataset to identify transcription factors important for accurately classifying

973 cells in pairwise comparisons of enhancers. Briefly, we ran randomForest independently

97410 times using the log-normalized TF-curated RPKM C1 data matrix, using 5,000 trees

975 per forest, for each of the pairwise comparisons (MGE-hs1056-M vs dMGE-hs1538-M; 
976 MGE-hs192-PM vs MGE-hs799-PM; CGE-hs841-M vs LGE-hs841-M; and CGE-hs953-

977 PM vs LGE-hs599-PM). We extracted the out-of-box error rates for all forests in addition

978 to the mean decrease in accuracy (MDA) and mean decrease in node impurity (MDNI)

979 scores for all genes, and calculated mean and standard deviation. Genes were ranked

980 by the mean MDA and MDNI scores.

981

982 Gene ontology analysis

983 We used the R package topGO (v2.36.0) (Alexa and Rahnenfuhrer, 2020) to perform

984 gene ontology (GO) analysis by TF-based cluster and enhancer group on DE genes in

985 the 10x dataset. Mouse GO data were downloaded from Bioconductor (org.Mm.eg.db)

986 (Carlson, 2019). We restricted analysis to GO Biological Process annotations and

987 required a minimal node size of greater than 20 . We used the 'weight01' framework and

988 Fisher test to define significant terms $(p$-value $<0.05)$. The background for all

989 enrichment comparisons is all 18,088 expressed genes. For Figure 5, clustering of

990 selected significant GO terms was performed using the R package pheatmap (Kolde,

991 2015) using hclust and default parameters.

992

\section{Full transcriptome analysis}

994 We repeated clustering analysis of single cells using the full transcriptome for both C1

995 and 10x scRNA-seq datasets. To define highly variable genes (HVGs), we calculated

996 the mean of all expressed genes in the Seurat object data slot using the Seurat function

997 FindVariableGenes using default parameters. The top 3,000 HVGs were used for

998 dimensionality reduction using PCA and UMAP visualization. For C1 data, we used the 
999 first 10 PCs to define 11 clusters using FindNeighbors (using k.param = 15, nn.method

1000 = "rann", annoy.metric = "euclidean") and FindClusters (using resolution = 1, algorithm =

10012 , group.singletons = F). For 10x data, we used the first 17 PCs to define 17 clusters

1002 using FindNeighbors (using k.param = 20, nn.method = "rann", annoy.metric =

1003 "euclidean") and FindClusters (using resolution = 1, algorithm = 2, group.singletons =

1004 F). UMAP visualization was generated using RunUMAP with the same parameters

1005 described for each dataset above. DE analysis was performed across clusters as

1006 described above.

1007

1008 Combined analysis of C1 and 10x datasets

1009 We used canonical correlation analysis (CCA) to combine the C1 and 10x datasets

1010 using both TF-curated and full transcriptome datasets. Briefly, the raw counts for both

1011 datasets were merged together, excluding genes that exhibited expression only in one

1012 dataset, and normalized using NormalizeData (normalization.method = "LogNormalize",

1013 scale.factor = 1e6). Integration anchors between both datasets were identified using

1014 FindIntegrationAnchors $($ reduction $=$ "cca", dims = 1:50, normalization.method $=$

1015 "LogNormalize", anchor.features = 3000). Datasets were integrated using 50 CCA

1016 components from FindIntegrationAnchors with IntegrateData (dims = 1:50), and center-

1017 scaled data generated using ScaleData. The integrated dataset was used for principal

1018 components analysis and the first 15 PCs were used to define 17 clusters using

1019 FindNeighbors (using k.param = 5, nn.method = "rann", annoy.metric = "euclidean") and

1020 FindClusters (using resolution $=1$, algorithm $=2$, group.singletons $=\mathrm{F}$ ). UMAP 
1021 visualization was generated using RunUMAP (reduction = "pca", n.neighbors = 20,

1022 n.epochs $=1000$, negative. sample.rate $=10)$.

1024 ABA ISH transcription factor scoring

1025 Expression levels of all transcription factors in the Allen Developing Mouse Brain Atlas

1026 (Lein et al., 2007) were estimated in subdomains of the subpallium for two ages: E11.5

1027 and E13.5. The subpallium was divided into 4 anatomical domains (LGE, MGE,

1028 Septum, and POA). The LGE and MGE were further subdivided into laminar

1029 subdomains: VZ/SVZ and MZ for E11.5; VZ, SVZ1, SVZ2, and MZ for E13.5.

1030 Expression was annotated from sagittal sections in each subdomain as an Intensity

1031 value and a Density value on a 0-5 scale. The intensity value was derived from the

1032 color-coded intensity viewing option called "expression mask" on the ABA. The density

1033 value was assigned by binning the frequency of individual signals across subdomains

1034 onto the $0-5$ scale.

1035

1036 Histology

1037 Section preparation.

1038 Pregnant dams were sacrificed by $\mathrm{CO}_{2}$ inhalation, confirmed by cervical dislocation.

1039 E11.5 embryos were removed and placed into ice-cold 1x PBS. The heads were cut off

1040 and drop-fixed in 4\% paraformaldehyde in PBS overnight at $4{ }^{\circ} \mathrm{C}$. The fixed heads were

1041 then cryoprotected in $20 \%$ sucrose in PBS overnight at $4{ }^{\circ} \mathrm{C}$, embedded in OCT

1042 compound (TissueTek) and frozen on dry ice. Cryostat sections $15 \mu \mathrm{m}$ thick were cut

1043 directly onto SuperFrost slides and allowed to dry. 
1045 Fluorescent immunohistochemistry.

1046 Sections were rinsed in PBS, blocked for 1 hour at room temperature in PBST (PBS +

$10470.25 \%$ Triton X-100) with $10 \%$ FBS, and incubated with primary antibody diluted in

1048 PBST $+10 \%$ FBS overnight at $4{ }^{\circ} \mathrm{C}$. Primary antibodies used were mouse anti-Tle 4

1049 (1:100, Santa Cruz Biotechnology, \#sc-365406, RRID: AB_10841582), rat anti-GFP

1050 (1:1000, Nacalai Tesque, \#04404-84, RRID: AB_10013361) and rabbit anti-GFP

1051 (1:5000, Abcam, \#ab6556, RRID: AB_305564). The following day, sections were

1052 washed $2 \times 15 \mathrm{~min}$ in PBST and $2 \times 15 \mathrm{~min}$ in PBS, incubated for 2 hours at room

1053 temperature with Alexa fluor-conjugated secondary antibodies (1:750, Invitrogen).

1054 Finally, sections were washed $2 \times 15 \mathrm{~min}$ in PBST and $2 \times 15 \mathrm{~min}$ in PBS and

1055 coverslipped with Fluorescence Mounting Medium (DAKO \#S3023).

1057 Fluorescence in situ hybridization (FISH).

1058 The antisense RNA probes used in this study have been described previously (Long et

1059 al., 2009). In situ hybridization was performed on $15 \mu \mathrm{m}$ cryostat sections as described

1060 previously (Lindtner et al., 2019) up until the antibody blocking step, with the addition of

1061 a peroxidase quenching step for 20 minutes in $3 \% \mathrm{H}_{2} \mathrm{O}_{2}$ in $\mathrm{PBS}$ after the SSC washes.

1062 After blocking, slides were incubated with anti-Digoxigenin-POD Fab fragments (Roche

$1063 \# 11207733910$ ) diluted 1:500 in NTT blocking buffer for 1 hour at room temperature.

1064 Slides were then washed for $3 \times 5$ minutes in NTT and developed with Cy5-Tyramide

1065 signal amplification reagent (TSA Plus Kit, Akoya Biosciences \#NEL745001KT)

1066 according to the manufacturer's instructions. Finally, sections were washed $3 \times 15$ 
1067 minutes in PBS, incubated for 5 minutes with nuclear counterstain Hoechst 33342

1068 (1:1000 in PBS, ThermoFisher \#H3570), rinsed $3 \times 5$ minutes in PBS, and coverslipped

1069 with Fluorescence Mounting Medium (DAKO \#S3023).

1070

1071 Imaging.

1072 Low-magnification epifluorescent images were taken using a Coolsnap camera

1073 (Photometrics) mounted on a Nikon Eclipse 80i microscope using NIS Elements

1074 acquisition software (Nikon) and a 4x or 10x objective. Confocal images were taken with

$107520 x$ air and 40x oil objectives on an Andor Borealis CSU-W1 spinning disk confocal

1076 mounted on a Nikon Ti Microscope and captured with an Andor Zyla sCMOS camera

1077 and Micro-Manager software (Open Imaging). The raw images were pre-processed with

1078 ImageJ software (v2.0.0) to adjust brightness/contrast and convert to 8-bit RGB.

1079 Confocal images were stitched laterally to create composites using the Grid/Collection

1080 stitching ImageJ plugin with linear blending (Preibisch et al., 2009).

1081

1082 Cell counts.

1083 Cell counting was performed on single confocal image planes in ImageJ using the Cell

1084 Counter plugin. Counts were summed from at least 3 rostrocaudal sections for each

1085 brain. Tle4-positive cells were counted first with the GFP channel hidden, excluding

1086 cells in the VZ (designated by nuclear staining), then scored as positive or negative for

1087 overlap with GFP staining. 


\section{Supplementary Figures}
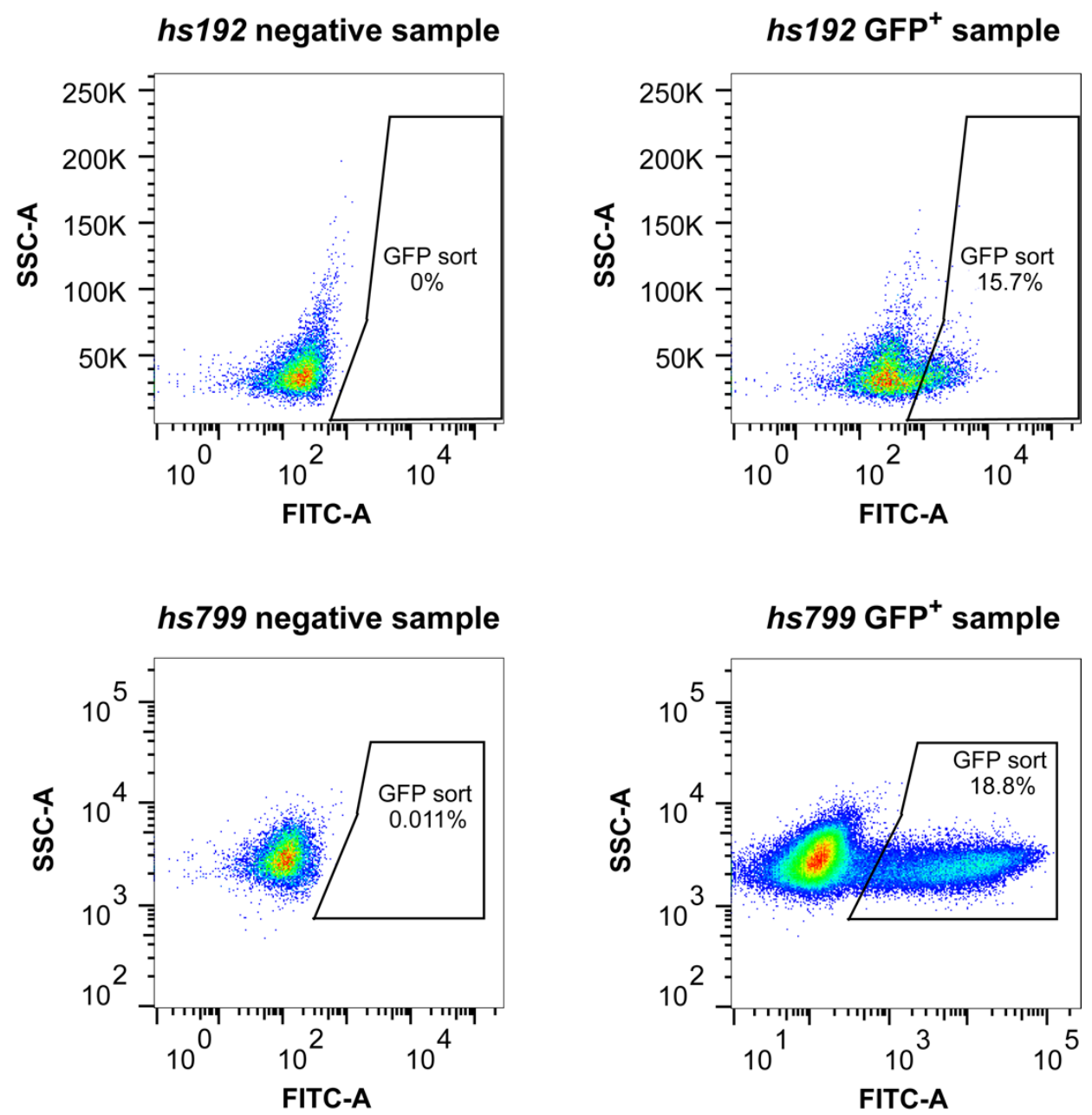

1092

1093 Supplementary Figure 1: FACS gating schematics.

1094 Density plots showing side-scatter (SSC) versus GFP fluorescence intensity (FITC) for

1095 negative and GFP-positive samples from $h s 192$ and $h s 799$ MGE. GFP sort area shows

1096 cells collected for scRNA-seq and the percentage of live cells captured. Negative

1097 samples were used to set the gating. $h s 799^{+}$cells show brighter GFP fluorescence than

$1098 h^{h} 192^{+}$cells. 
bioRxiv preprint doi: https://doi.org/10.1101/2021.01.11.426285; this version posted January 12, 2021. The copyright holder for this preprint (which was not certified by peer review) is the author/funder, who has granted bioRxiv a license to display the preprint in perpetuity. It is made available under aCC-BY-NC-ND 4.0 International license.
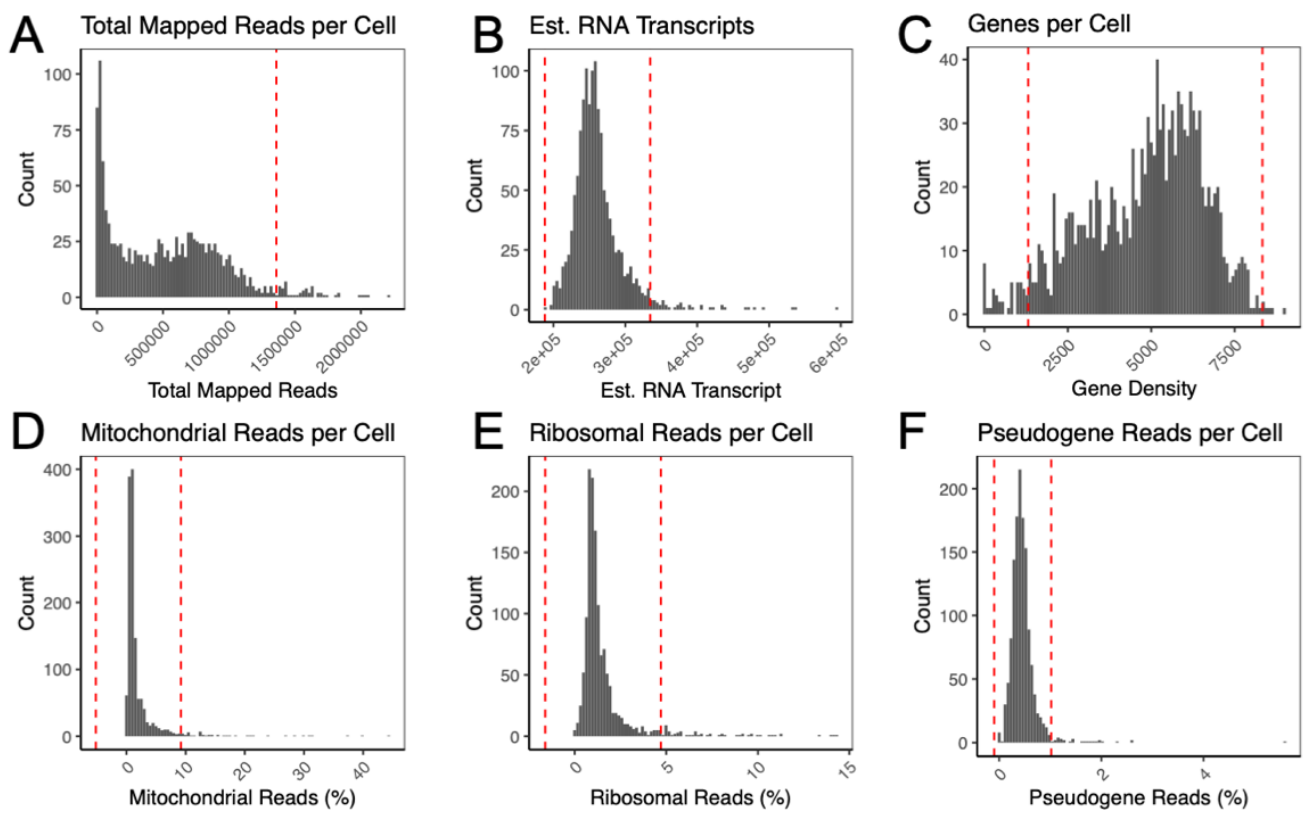

G

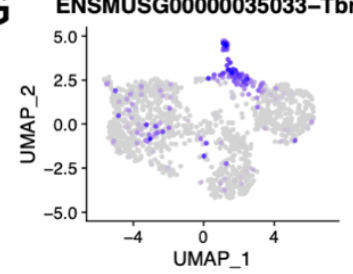

ENSMUSG00000029546-Uncx

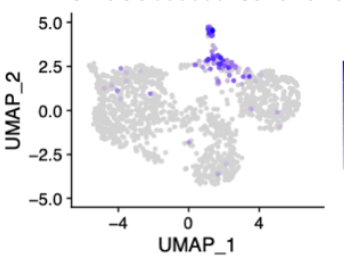

I

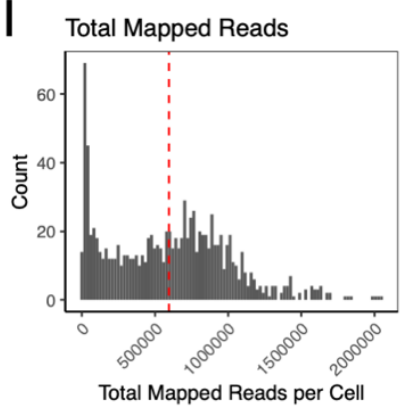

ENSMUSG00000019230-Lhx9

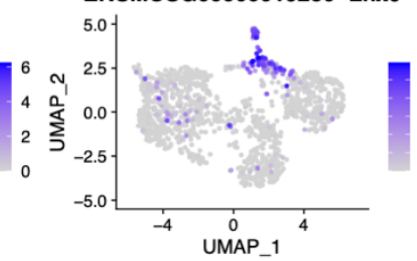

ENSMUSG00000048540-Nhlh2

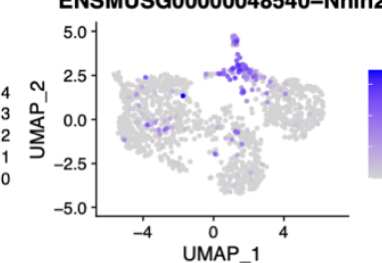

$\mathrm{J}$ Est. RNA Transcripts $\mathrm{K}$

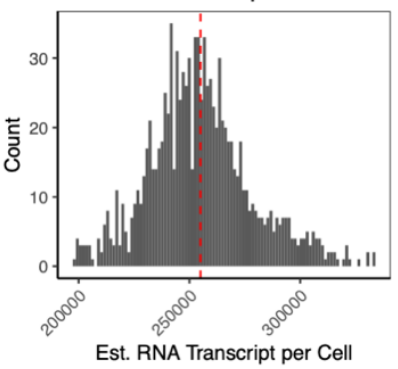

K Genes per Cell

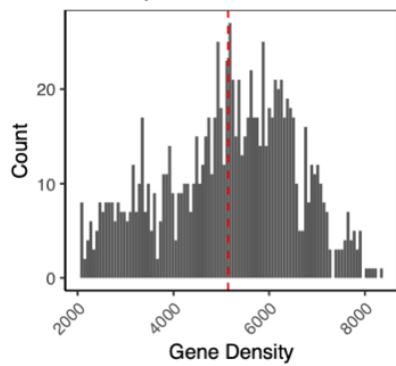

$\mathrm{H}$

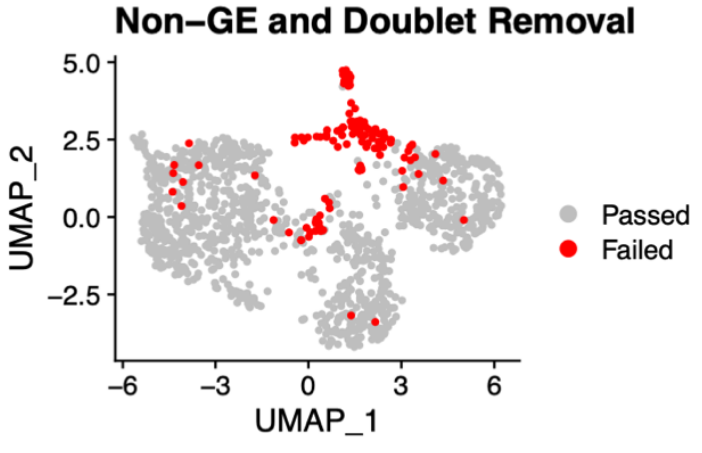

M Transgene Expression by FACS
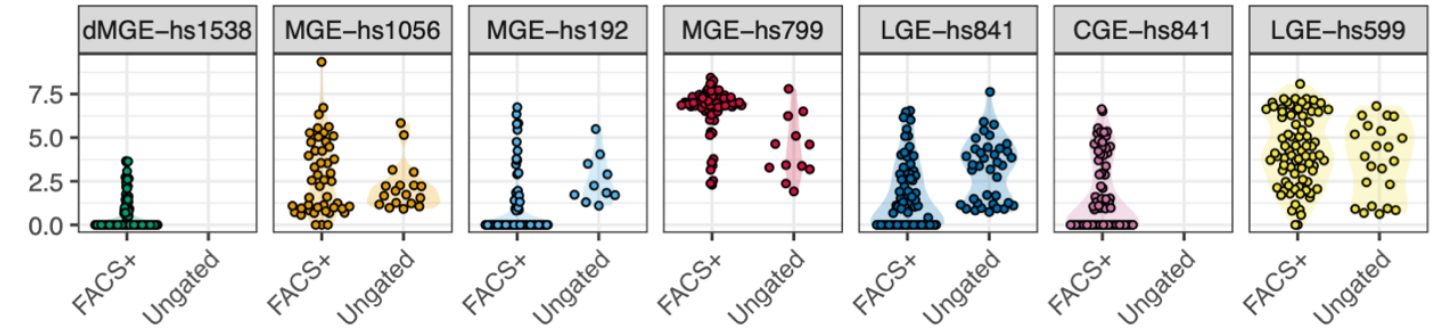

L Mitochondrial Reads per Cell

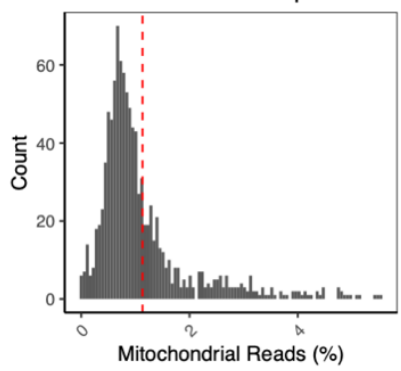




\section{Supplemental Figure 2: C1 scRNA-seq quality control.}

1101 (A-F) Histogram of total uniquely mapped reads (A), estimated RNA transcript (B),

1102 number of expressed genes (C), percent mitochondrial reads (D), percent ribosomal

1103 reads $(\mathbf{E})$, and percent pseudogenes $(\mathbf{F})$ per cell, post-duplication removal for all

1104 sequenced samples. Cells above or below the red lines were removed from

1105 downstream analysis. Red lines represent mean \pm 2 s.d. (G) UMAP of transcription-

1106 factor-curated clusters after removal of cells flagged in A-F, colored by normalized

1107 expression of four genes used for flagging cells as non-ganglionic in origin (Tbr1, Lhx9,

1108 Uncx, and Nh/h2). (H) UMAP colored by quality control flag for contaminating cells and

1109 suspected doublets (passed: used for downstream analysis; failed: removed from

1110 downstream analysis). (I-L) Histograms of total mapped reads (I), estimated RNA

1111 transcripts $(\mathbf{J})$, expressed genes per cell $(\mathbf{K})$, and percent mitochondrial reads per cell

1112 (L), for all cells that passed final quality control. Red line represents mean. (M)

1113 Normalized transgene expression for each of the seven enhancers profiled, separated

1114 by FACS ${ }^{+}$gating or no $\mathrm{FACS}^{+}$gating (if performed). 
bioRxiv preprint doi: https://doi.org/10.1101/2021.01.11.426285; this version posted January 12, 2021. The copyright holder for this preprint (which was not certified by peer review) is the author/funder, who has granted bioRxiv a license to display the preprint in perpetuity. It is made available under aCC-BY-NC-ND 4.0 International license.

HVG $(n=3000)$ clustering by cell cycle phase, dissection, and sequencing lane without cell cycle regression

A

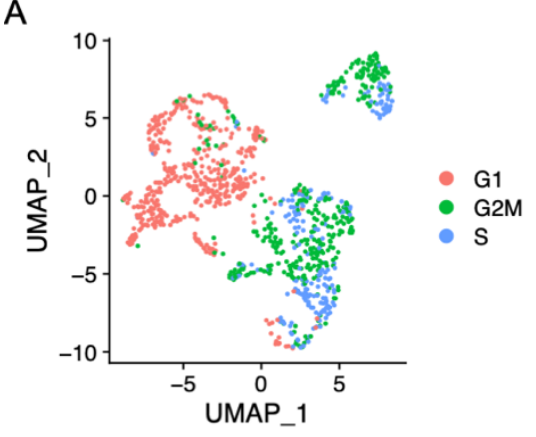

B

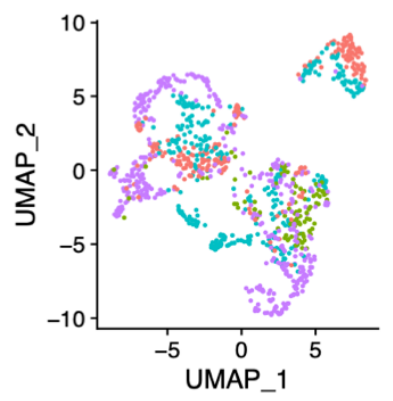

C

- CGE

- dMGE

- LGE

- MGE

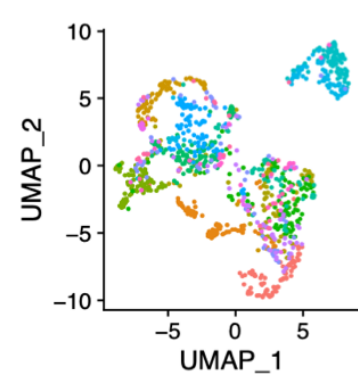

- BGSC01

- BGSC02

- BGSC03

- BGSC04

- BGSC05

- BGSC06

- BGSC07

- BGSC08

- BGSC09

- BGSC10

- BGSC11

- BGSCL2

- BGSCL3

- BGSCL4

RUB150

- RUB201

- BGSC01

- BGSC02

- BGSC03

- BGSC04

- BGSC05

- BGSC06

- BGSC07

- BGSC08

- BGSC09

- BGSC10

- BGSC11

- BGSCL2

- BGSCL3

- BGSCL4

- RUB150

- RUB201

\section{$G_{\text {ENSMUSG00000001496-Nkx2-1 }}$}

ENSMUSG00000027168-Pax6

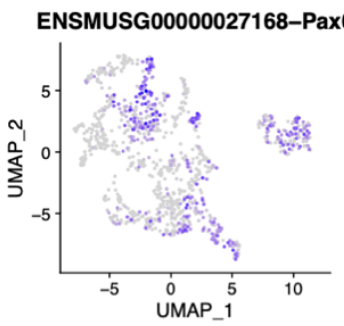

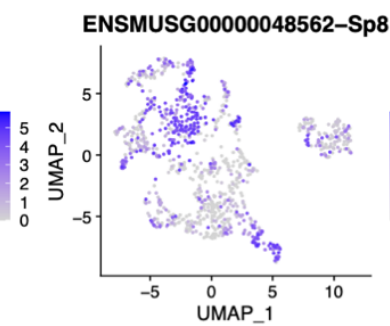

ENSMUSG00000069171-Nr2f1

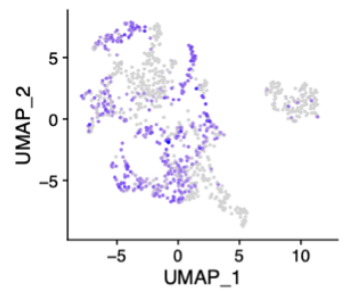

$\mathrm{H}$

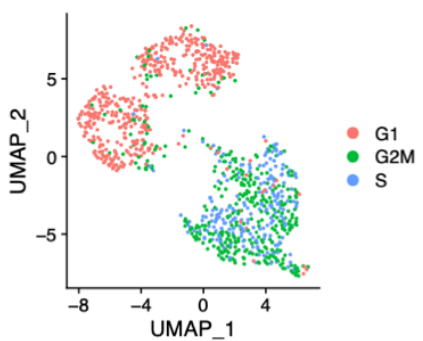

I

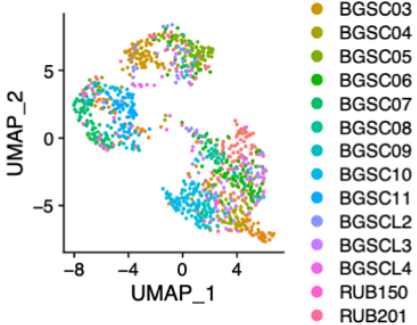

J

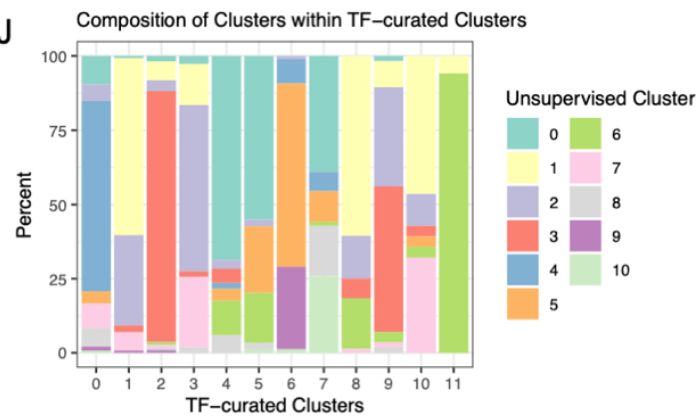

K

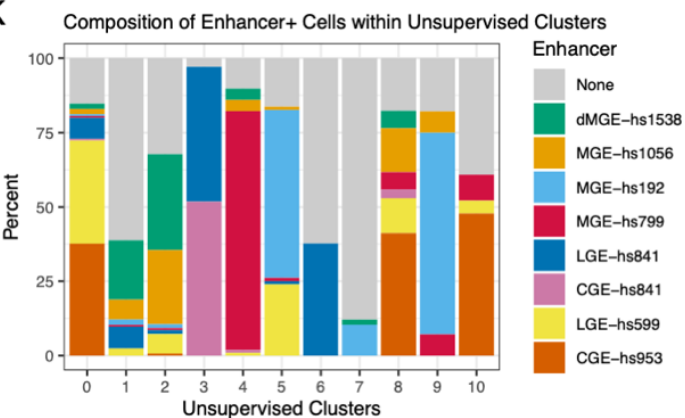


1117 Supplemental Figure 3: Comparison between full transcriptome and TF-curated

1118 analyses for C1 scRNA-seq.

1119 (A-C) UMAP representations using full transcriptome ("unsupervised") clustering of the

1120 top 3,000 highly variable genes (HVGs) with no cell cycle phase regression, colored by

1121 cell cycle phase $(\mathbf{A})$, region of dissection (B), and sequencing lane (C). (D-F) UMAP

1122 representations using full transcriptome of the top 3,000 highly variable genes (HVGs)

1123 after cell cycle phase regression, colored by cell cycle phase (D), region of dissection

$1124(\mathbf{E})$, and sequencing lane $(\mathbf{F}) .(\mathbf{G})$ UMAP colored by normalized expression of four

1125 region-defining transcription factors (Nkx2-1, Pax6, Sp8, and Nr2f1). (H) TF-curated

1126 UMAP colored by cell cycle phase. (I) TF-curated UMAP colored by sequencing lane.

1127 (J) Bar plot of TF-curated clusters by percent representation from unsupervised (full

1128 transcriptome) clusters. Some unsupervised clusters (e.g. cl-10, light green) remain

1129 consistent between clustering methods, while others (e.g. cl-1, light yellow) are split

1130 across multiple TF-curated clusters. (K) Bar plot of unsupervised clusters by enhancer

1131 representation. Some enhancers (e.g. MGE-hs799, red) are relatively consistent across

1132 clustering methods, while others (e.g. MGE-hs192, light blue) split across clusters. 
bioRxiv preprint doi: https://doi.org/10.1101/2021.01.11.426285; this version posted January 12,2021 . The copyright holder for this preprint (which was not certified by peer review) is the author/funder, who has granted bioRxiv a license to display the preprint in perpetuity. It is made available under aCC-BY-NC-ND 4.0 International license.

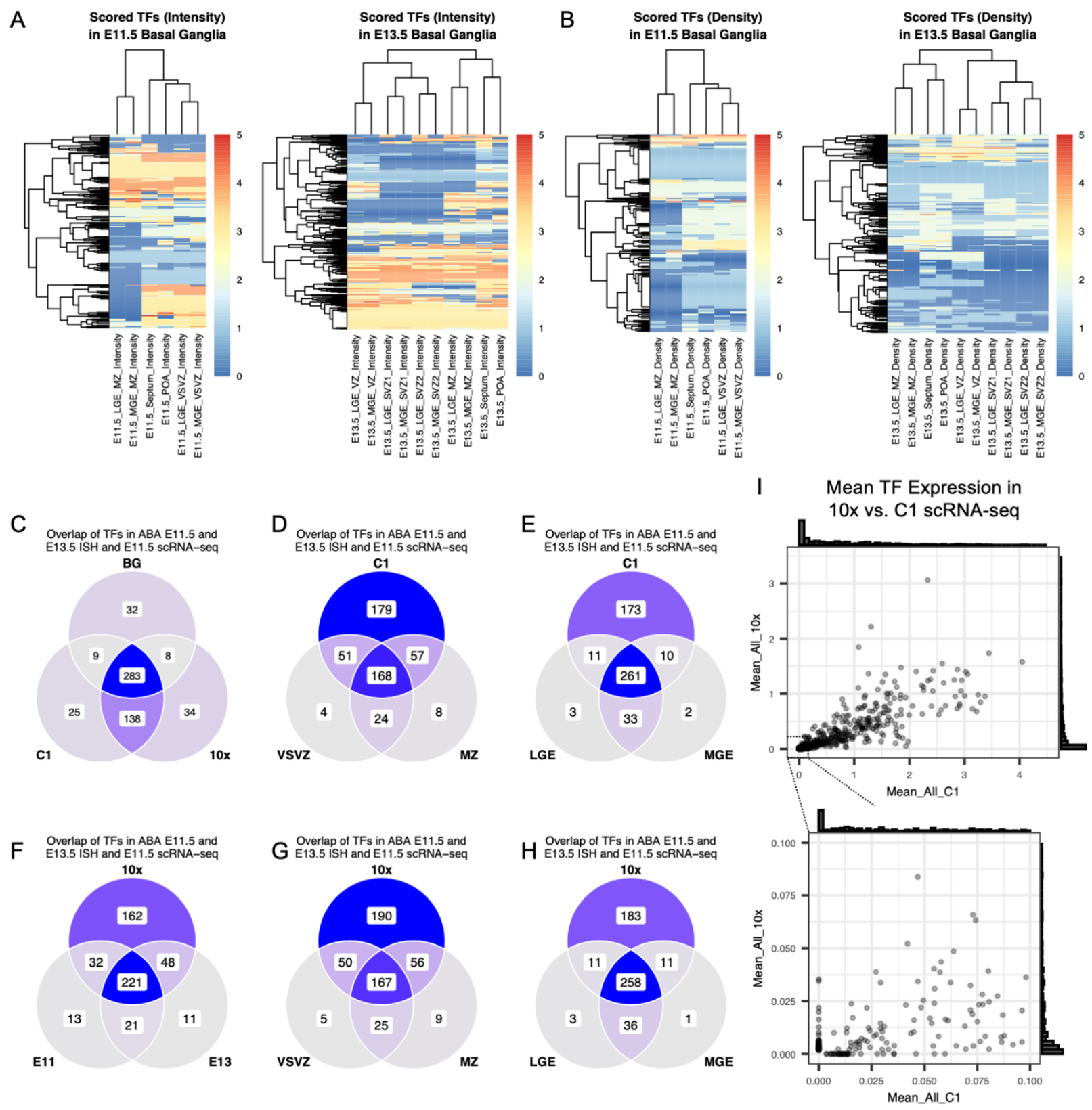

1136 Supplemental Figure 4: Allen Developing Mouse Brain Atlas and scRNA-seq

1137 overlaps.

1138 (A) Heatmap for E11.5 (left) and E13.5 (right) scored transcription factor (TF)

1139 expression by intensity in Allen Developing Mouse Brain Atlas (ABA) RNA in situ

1140 hybridization (ISH) for 689 TFs. Rows: transcription factors; columns: areas and time 
1141 points scored. MGE: medial ganglionic eminence; LGE: lateral ganglionic eminence;

1142 POA: preoptic area; VSVZ: ventricular/subventricular zone; MZ: mantle zone. (B)

1143 Heatmap of scored expression as in (A), by density. (C) Venn diagram of overlap of TFs

1144 with non-zero intensity score in E11.5 and E13.5 basal ganglia (MGE and LGE

1145 combined) from the ABA dataset ("BG") to detected TFs in C1 and 10x scRNA-seq

1146 datasets. (D-E) Overlap of TFs expressed in C1 scRNA-seq, separated by non-zero

1147 VSVZ vs MZ intensity scores (D) or LGE vs MGE scores (E) in E11.5 and E13.5 ABA

1148 ISH. (F) Overlap of TFs expressed in 10x scRNA-seq, separated by non-zero intensity

1149 scores in E11.5 vs E13.5 ABA ISH. (G-H) Overlap of TFs expressed in 10x scRNA-seq,

1150 separated by non-zero VSVZ vs MZ intensity scores (G) or LGE vs MGE scores $(\mathbf{H})$ in

1151 E11.5 and E13.5 ABA ISH. (I) (Top) Plot comparing mean normalized expression of

1152 transcription factors between $10 x$ and $C 1$ scRNA-seq. Cell distribution along $x-$ and $y-$

1153 axes are represented by histograms. (Bottom) Enlargement of the bottom-left corner of

1154 the top panel, showing distribution of low-representation TFs and dataset-specific

1155 expression. 
bioRxiv preprint doi: https://doi.org/10.1101/2021.01.11.426285; this version posted January 12, 2021. The copyright holder for this preprint (which was not certified by peer review) is the author/funder, who has granted bioRxiv a license to display the preprint in perpetuity. It is made available under aCC-BY-NC-ND 4.0 International license.
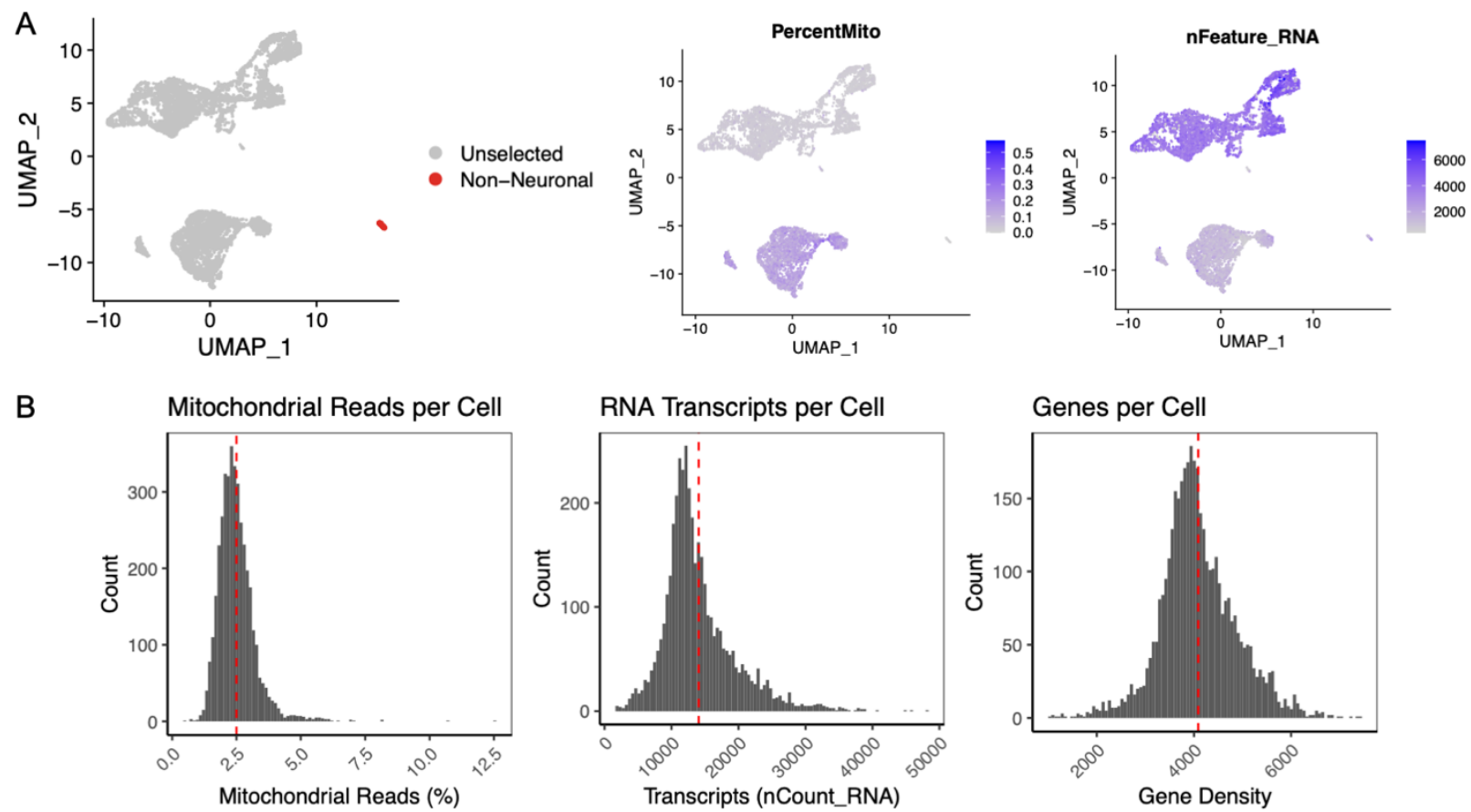

C Separation by Cell Cycle Phase and Barcode in Mitotic Cells using Highly Variable Genes
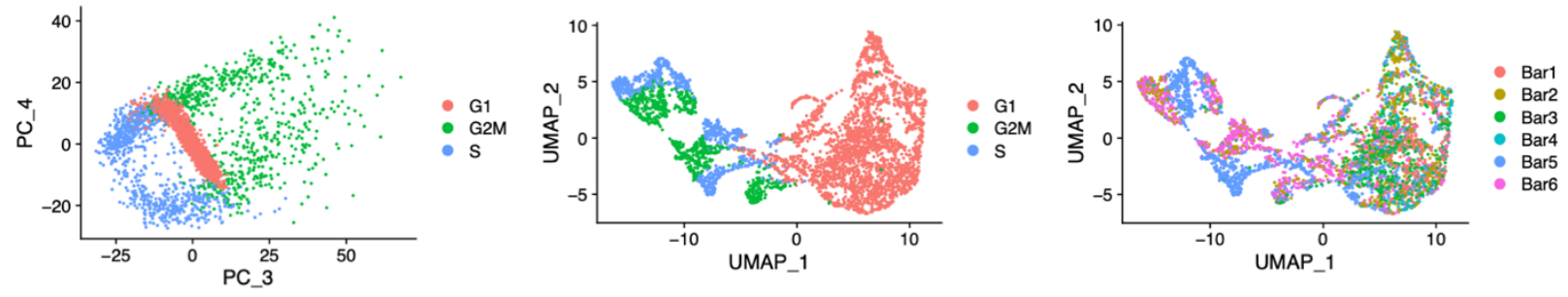

D Continued Separation by Cell Cycle Phase and Barcode post Cell Cycle Regression
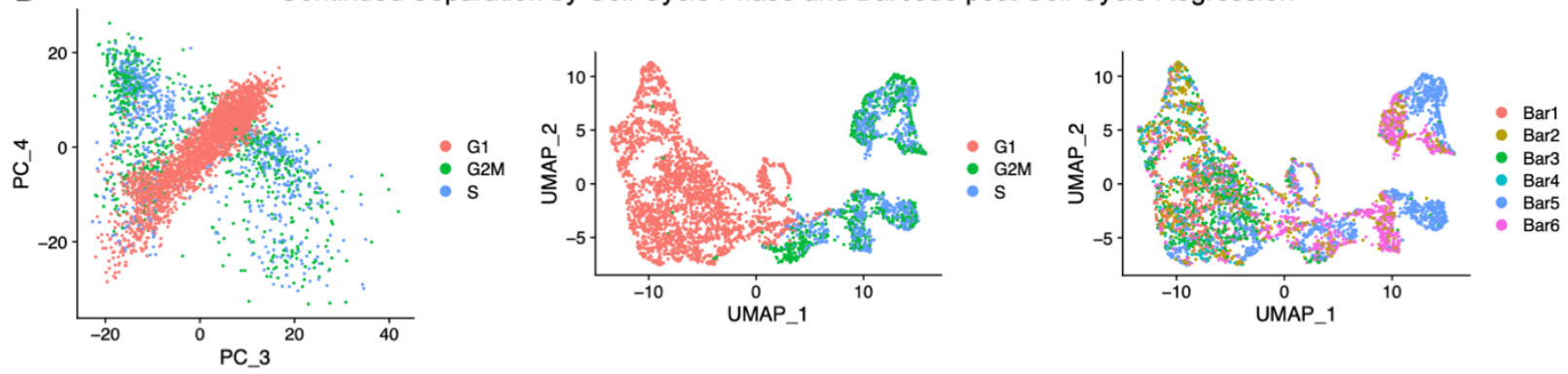

E Reduced Cell Cycle Phase and Barcode Separation using Transcription Factors Only
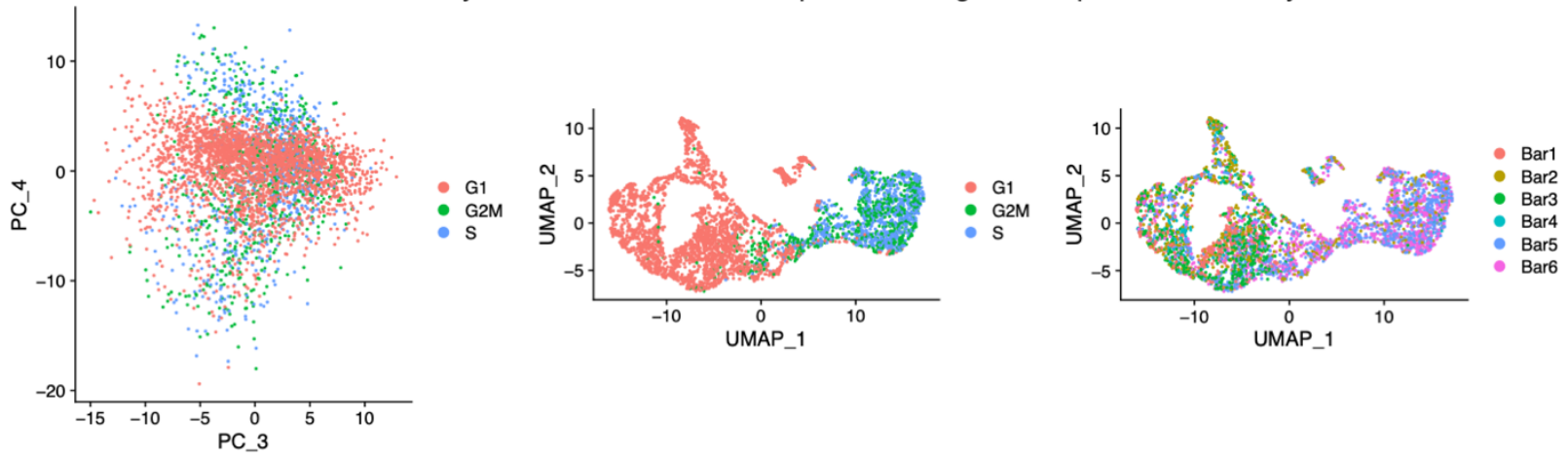


\section{Supplemental Figure 5: 10x scRNA-seq quality control.}

1158 (A) UMAP representation of single cells after sample demultiplexing and removal of

1159 multiplets using the MULTI-seq pipeline, colored by cells flagged for likely non-neuronal

1160 origin (left), percent mitochondrial reads, (middle), and number of expressed genes per

1161 cell (right). (B) Distribution of percent mitochondrial reads (left), estimated RNA

1162 transcripts (middle), and number of expressed genes per cell (right) with means (red

1163 line) after removal of all cells flagged by quality control metrics (effectively cells

1164 UMAP_2 $<0$ in A). (C) Principal components (PCs) 3 and 4 separate mitotic cells by cell

1165 cycle phase in full transcriptome (unsupervised) clustering using the top 3,000 highly

1166 variable genes (HVGs) (left). In UMAP representation of unsupervised clustering, cells

1167 separate by cell cycle phase (middle) and MULTI-seq barcode (right) in mitotic cells. (D)

1168 After cell cycle regression, cell cycle phase separation is negated (left and middle) but

1169 cells still separate by barcode in mitotic cells (right). (E) Using transcription factor-based

1170 clustering, both cell cycle phase separation (left and middle) and barcode separation

1171 (right) are negated. 
bioRxiv preprint doi: https://doi.org/10.1101/2021.01.11.426285; this version posted January 12, 2021. The copyright holder for this preprint (which was not certified by peer review) is the author/funder, who has granted bioRxiv a license to display the preprint in perpetuity. It is made available under aCC-BY-NC-ND 4.0 International license.
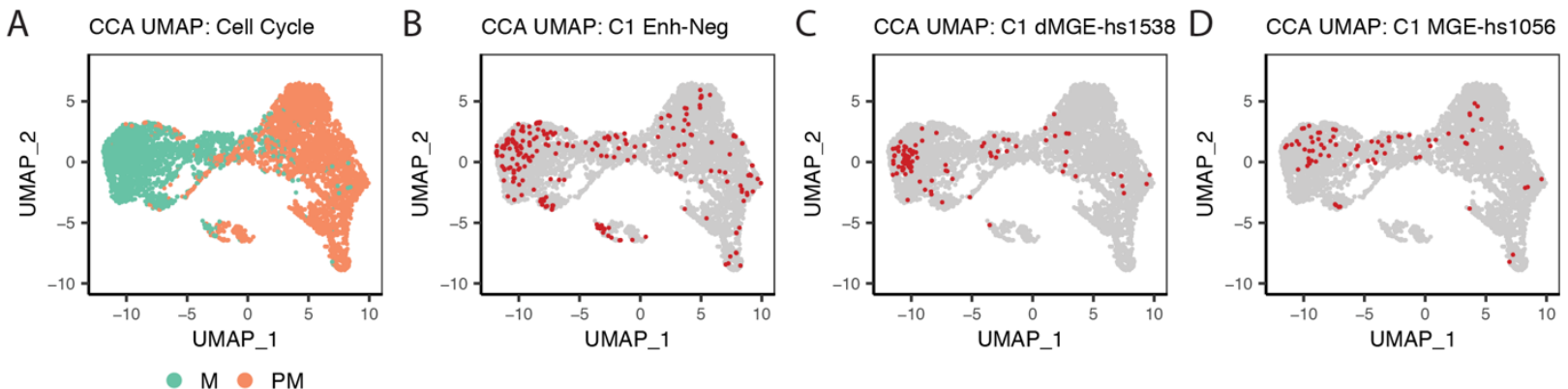

E CCA UMAP: C1 MGE-hs799

F CCA UMAP: 10x MGE-hs799

G
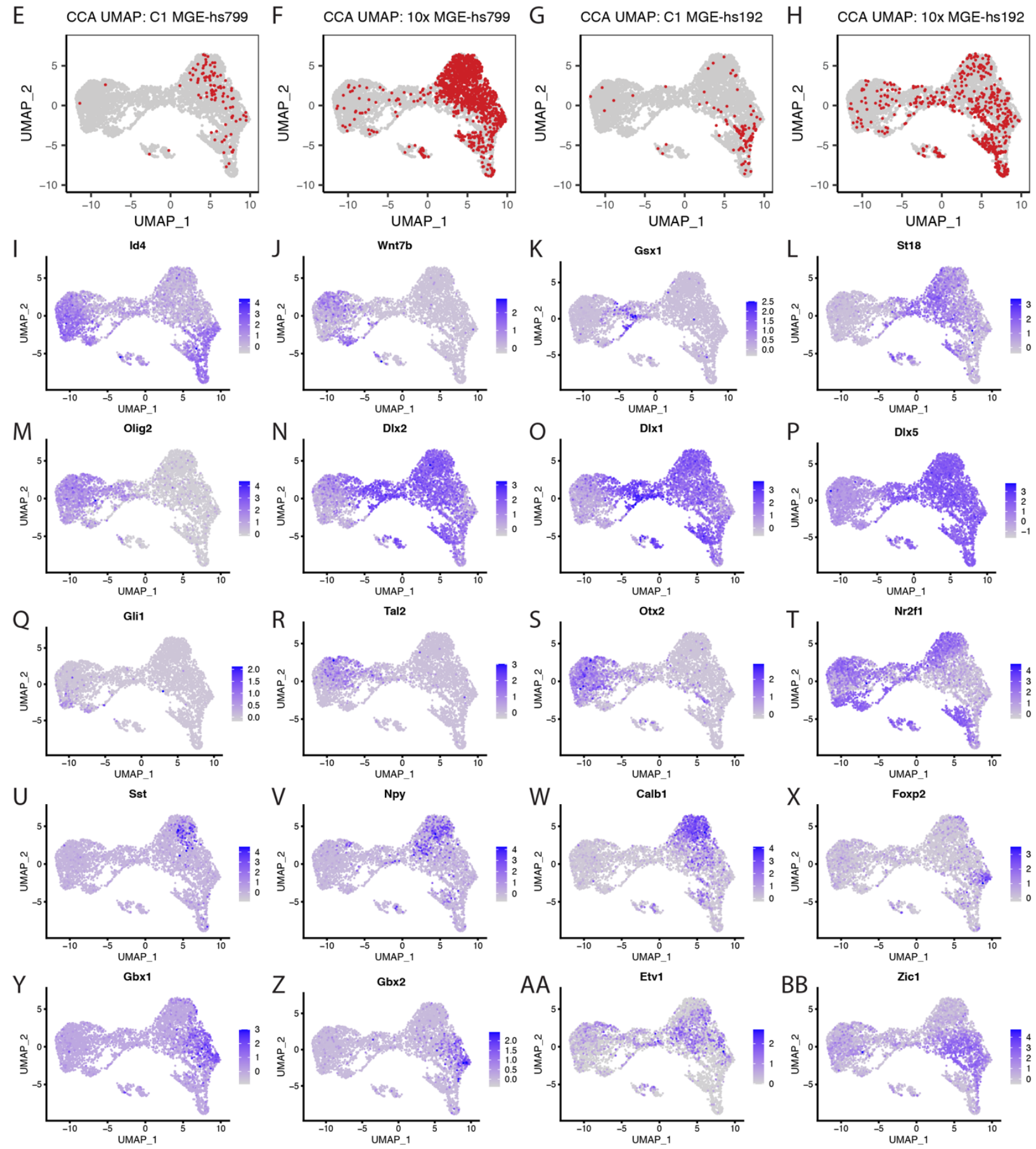
1173 Supplemental Figure 6: Canonical correlation analysis between C1 and 10x MGE

1174 scRNA-seq datasets.

1175 (A) TF-curated UMAP of combined C1 and 10x scRNA-seq using canonical correlation

1176 analysis, colored by mitotic state (green: mitotic; orange: postmitotic) (B-H) UMAP plots

1177 colored by C1 or 10x enhancer group. Red: selected group; grey: all other cells. (I-BB)

1178 UMAP plots of normalized gene expression for genes shown in Figure 5 (I-T) and

1179 Figure $6(\mathrm{U}-\mathrm{BB})$. 
bioRxiv preprint doi: https://doi.org/10.1101/2021.01.11.426285; this version posted January 12, 2021. The copyright holder for this preprint (which was not certified by peer review) is the author/funder, who has granted bioRxiv a license to display the preprint in perpetuity. It is made available under aCC-BY-NC-ND 4.0 International license.
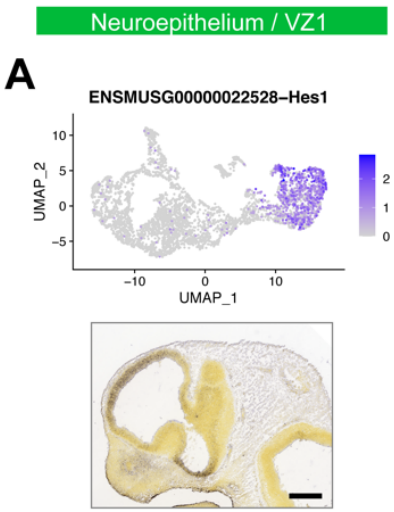

B
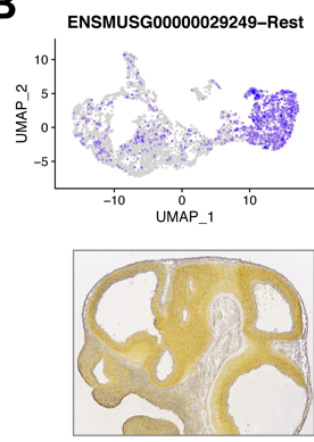

C
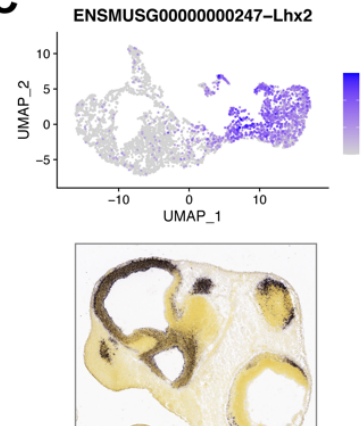

D

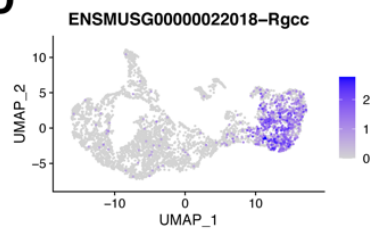

H
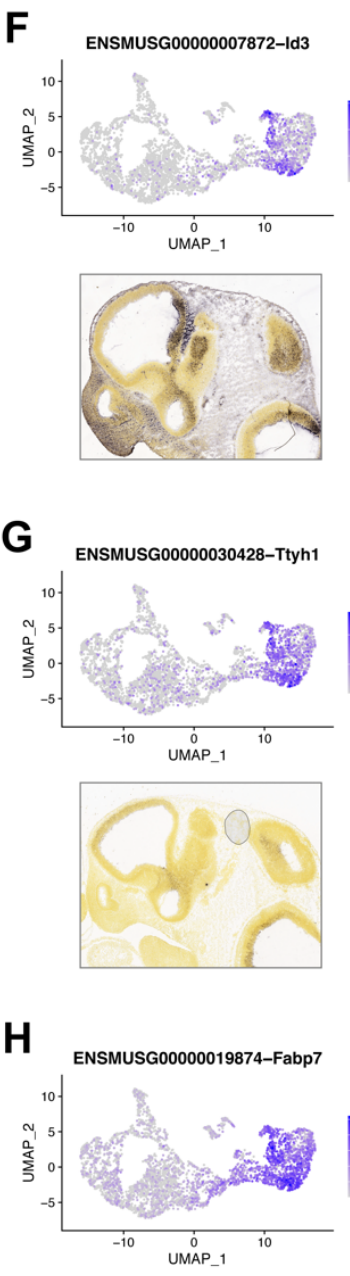

G
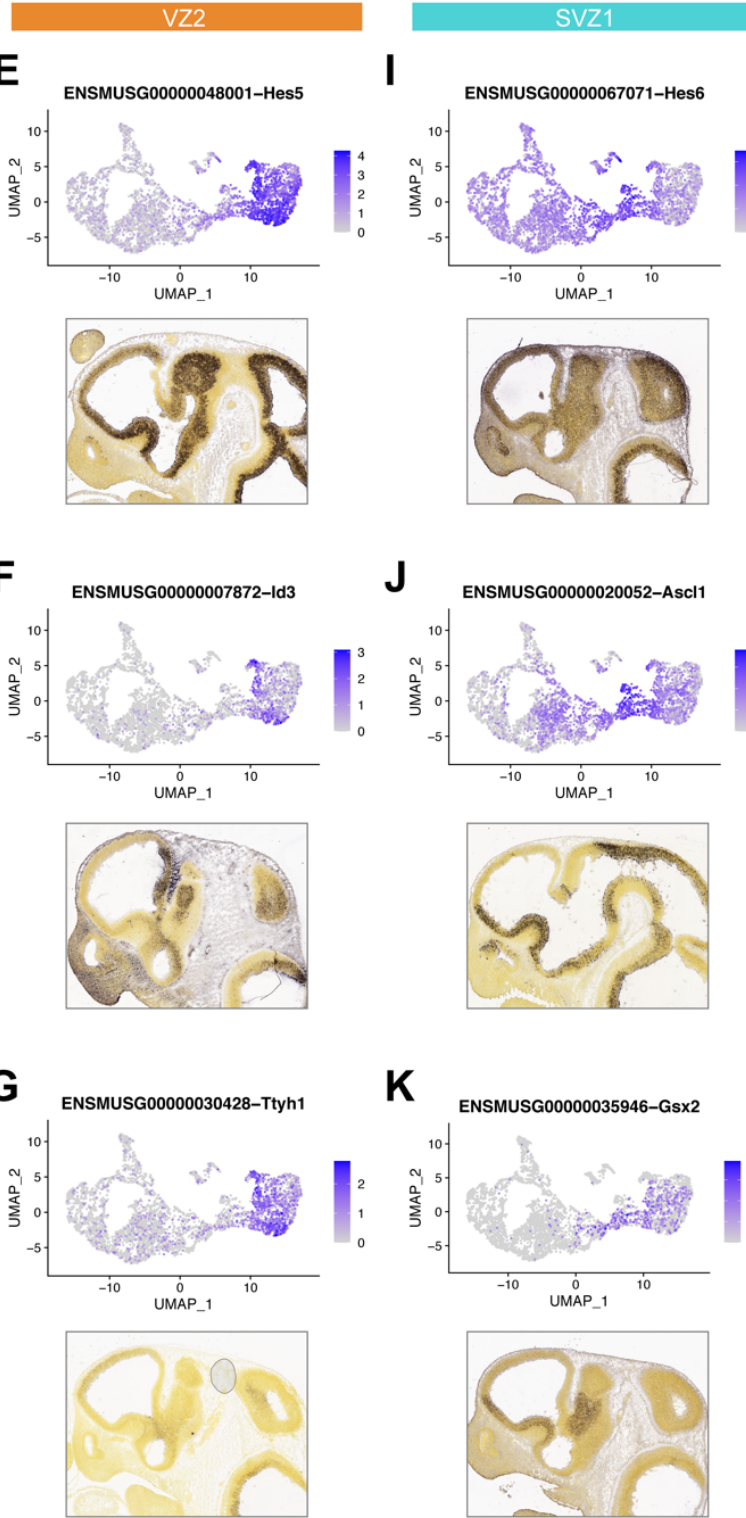

$\mathbf{J}$
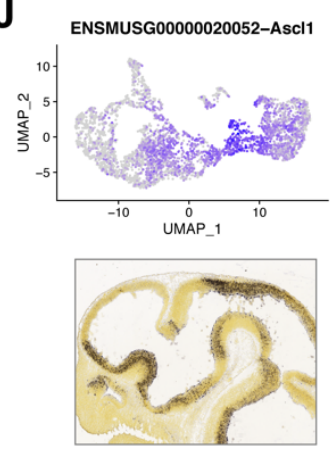

K
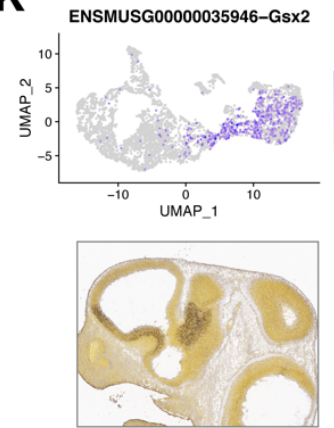

L
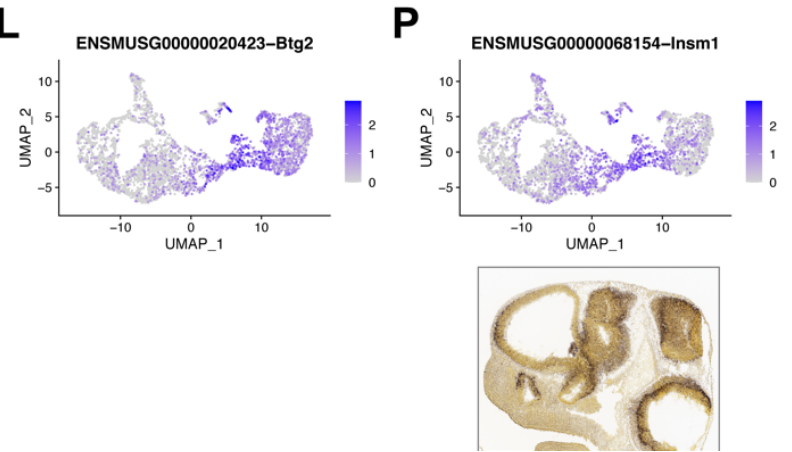

$\mathbf{M}$

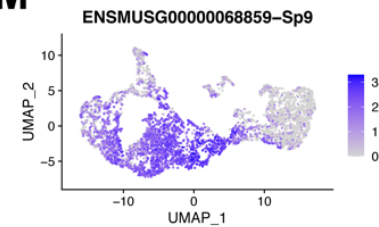

$\mathbf{N}$
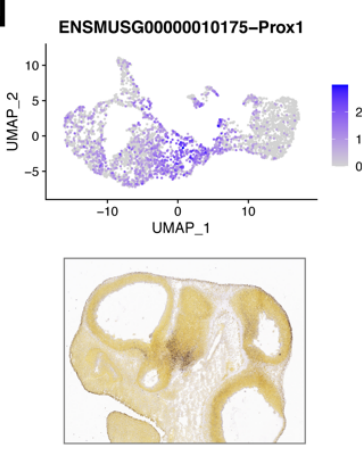

0
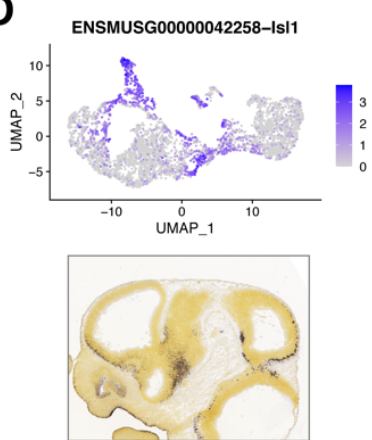

1181

1182 


\section{Supplementary Figure 7: Mitotic genes marking VZ/SVZ transitions.}

1184 (A-F) Gene expression UMAPs and representative ISH on E11.5 sagittal sections from

1185 the ABA showing expression of genes that correlate with developmental progression

1186 from early VZ1 (neuroepithelium) to late SVZ (SVZ2). Scale bar: $500 \mu \mathrm{m}$. 
bioRxiv preprint doi: https://doi.org/10.1101/2021.01.11.426285; this version posted January 12, 2021. The copyright holder for this preprint (which was not certified by peer review) is the author/funder, who has granted bioRxiv a license to display the preprint in perpetuity. It is made available under aCC-BY-NC-ND 4.0 International license.
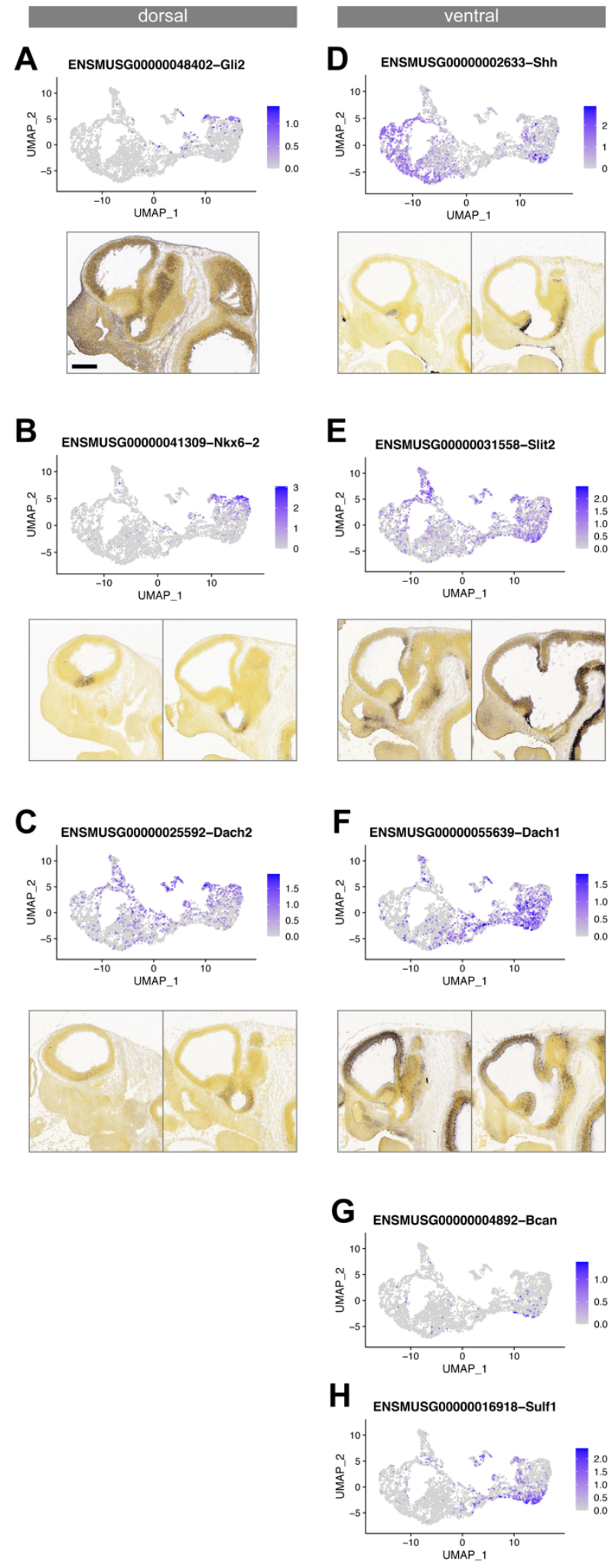
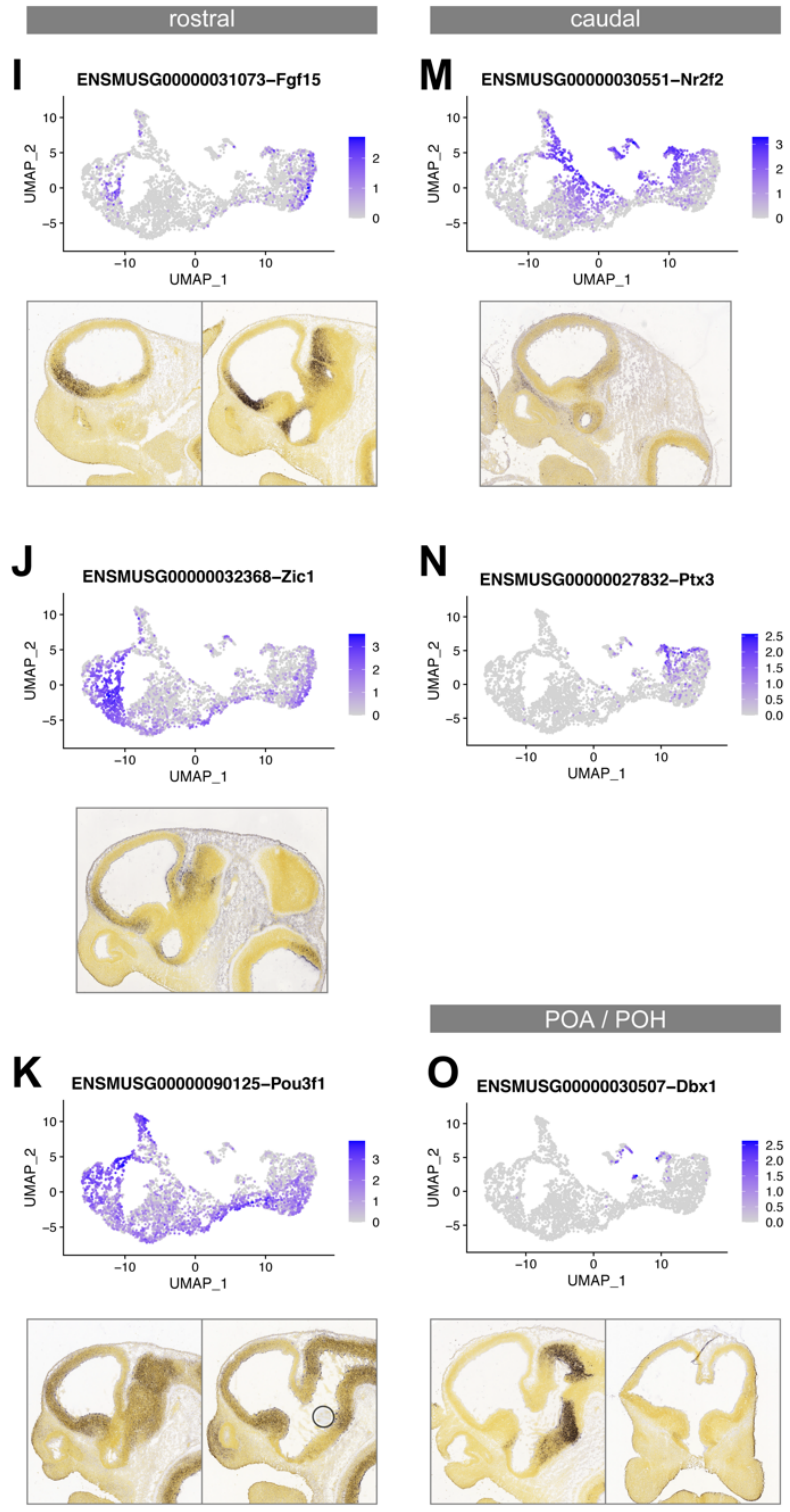

O ensmusG00000030507-Dbx1
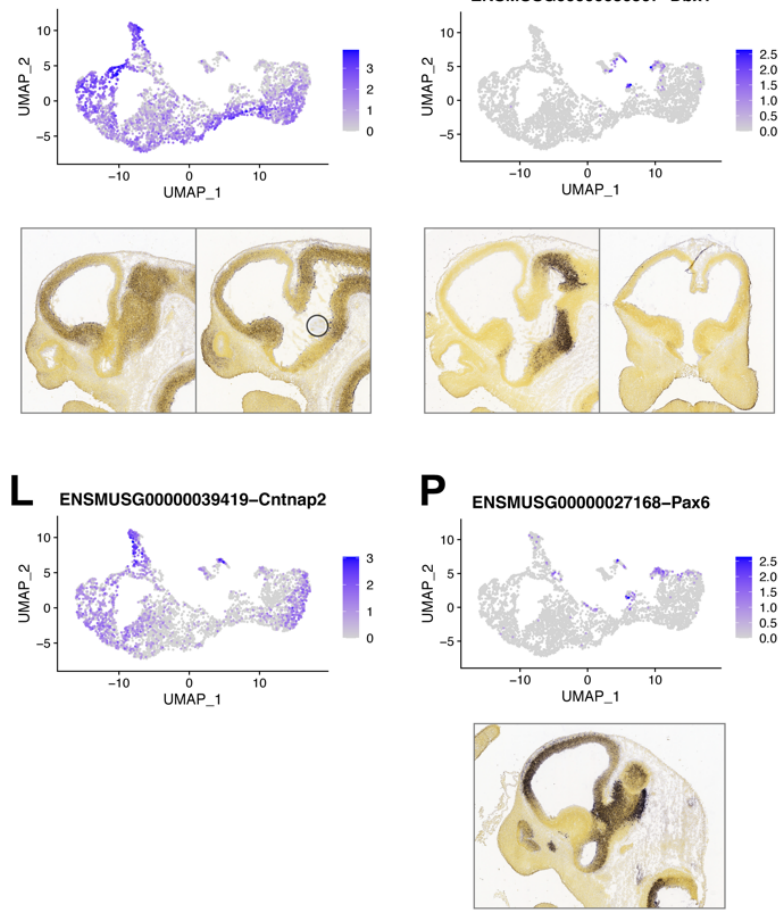
1189 Supplementary Figure 8: Mitotic genes with dorsoventral or rostrocaudal bias.

1190 (A-H) Gene expression UMAPs and representative ISH on E11.5 sagittal sections from

1191 the ABA showing genes with dorsal (Gli2, Nkx6-2, Dach2) or ventral (Shh, Slit2, Dach1,

1192 Bcan, Sulf1) bias in mitotic cells. Dach2, Dach1, Bcan and Sulf1 regional expression in

1193 the MGE has not been reported previously. (I-N) Gene expression UMAPs and

1194 representative ISH on E11.5 sagittal sections from the ABA showing genes with

1195 rostrocaudal bias in mitotic cells (Nr2f2, Ptx3, Fgf15, Cntnap2, Zic1, Pou3f1). (O,P)

1196 UMAP plots and ISH images of genes marking POA2 (Dbx1) or POH (Pax6) VZ cells.

1197 These cells are contiguous with the caudal MGE anatomically and in the UMAP. Scale

1198 bar: $500 \mu \mathrm{m}$. 
bioRxiv preprint doi: https://doi.org/10.1101/2021.01.11.426285; this version posted January 12, 2021. The copyright holder for this preprint (which was not certified by peer review) is the author/funder, who has granted bioRxiv a license to display the preprint in perpetuity. It is made available under aCC-BY-NC-ND 4.0 International license.
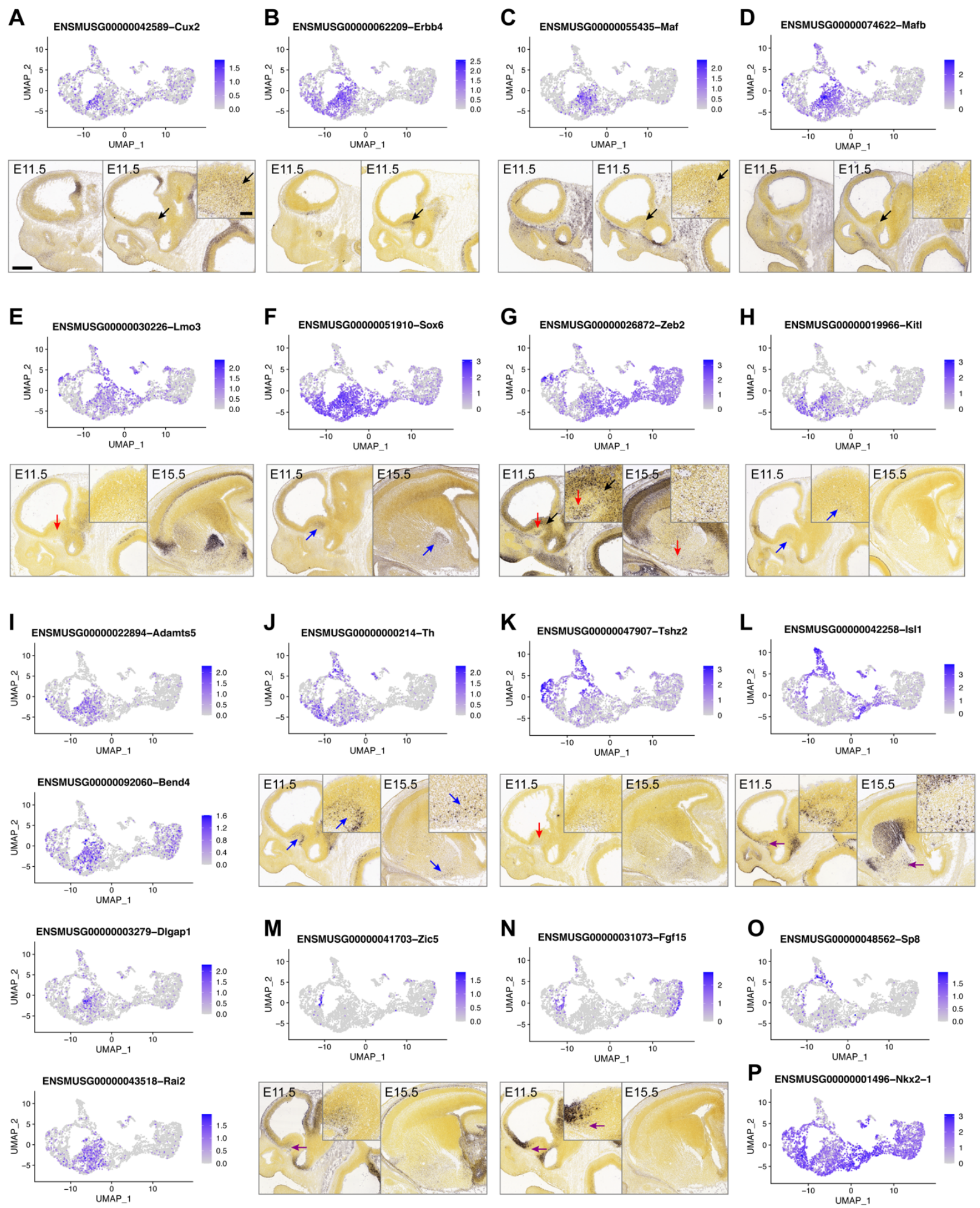


\section{Supplementary Figure 9: Genes marking classes of postmitotic neurons.}

1203 (A-D) Gene expression UMAPs and representative ISH on E11.5 sagittal sections from

1204 the ABA showing expression of markers for early CINs (Cux2, Erbb4, Maf, Mafb). (E-H)

1205 Gene expression UMAPs and representative ISH for genes marking CINs and classes

1206 of projection neurons (Lmo3, Sox6, Zeb2, Kitl). (I) Gene expression UMAPs of proposed

1207 novel early CIN markers (Adamts5, Bend4, Dlgap1, Rai2). (J-N) Gene expression

1208 UMAPs and representative ISH for genes marking classes of MGE-derived projection

1209 neurons (Th, Tshz2) or cholinergic neurons (Is/1, Zic5, Fgf15). (O,P) UMAPs of Sp8 and

$1210 N k \times 2-1$ showing the separation of cl-7 into an Sp8-positive non-MGE zone and an

1211 Nkx2-1-positive MGE-derived zone. Arrows indicate regions of higher magnification

1212 insets and cells of interest. Arrow colors: black, CINs; red, VP; blue, GP; purple; Ch.

1213 Scale bars: low magnification, $500 \mu \mathrm{m}$; high magnification insets, $100 \mu \mathrm{m}$. 


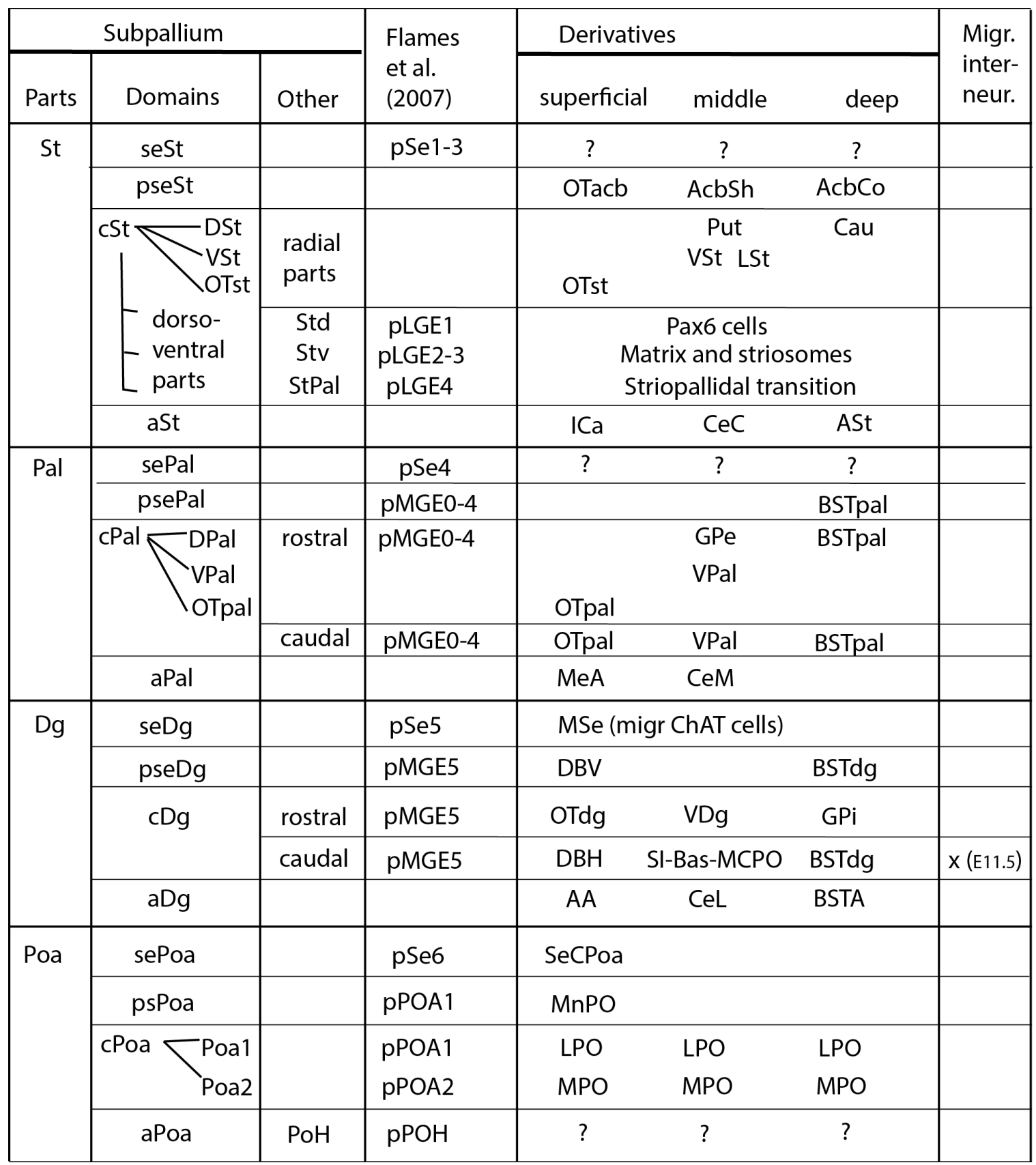

1216 Supplementary Table 6: Proposed origins of subpallial neurons.

1217 Proposed origins of subpallial neurons, based on a body of anatomical, developmental

1218 and genoarchitectural findings (Puelles et al., 2016, 2013; Silberberg et al., 2016). Here

1219 we compared the nomenclature from this paper with that from (Flames et al., 2007). The 
1220 left 3 columns (Subpallium) list the progenitor domains and subdomains: Column 1)

1221 Parts (major subdivisions): Striatum (St), Pallidum (Pal), Diagonal (Dg) and Preoptic

1222 Area (POA). Column 2) Domains (shared pattern across all the parts of septal,

1223 paraseptal, central and amygdalar regions, distinguished along the septoamygdalar

1224 axis): For St [septal St (seSt), paraseptal St (pseSt), central St (cSt), and amygdalar St

1225 (aSt)], for Pal [septal Pal (sePal), paraseptal Pal (psePal), central Pal (cPal), amygdalar

1226 Pal (aPal)], for Dg [septal Dg (seDg), paraseptal Dg (pseDg), central Dg (cDg), and

1227 amygdalar Dg (aDg)], and for Poa [septal Poa (sePoa), paraseptal Poa (psePoa),

1228 central Poa (cPoa), and amygdalar Poa (aPoa)]. Column 3) Other: provides

1229 morphological information on alternative subdivisions distinguished either along the

1230 radial dimension or the dorsoventral dimension. The central column lists the

1231 corresponding progenitor domains proposed in (Flames et al., 2007). Right columns

1232 (Derivatives) list the known neuronal derivatives of the subpallial progenitor domains,

1233 organized into radial (laminar) positions: superficial (closest to the pia), middle and deep

1234 (closest to the ventricle). The column on the far right (migrating interneurons) lists data

1235 from this publication proposing that the caudal Dg is the source for interneurons at

1236 E11.5. Other publications provide evidence that other subpallial progenitors also

1237 generate interneurons (for the pallium, striatum and olfactory bulb) at later ages.

1239 Other abbreviations, in the order that they are listed in the Table: OTacb (accumbens

1240 part of the olfactory tubercle), AcbSh (shell of the accumbens), AcbCo (core of the

1241 accumbens), OTst (central striatal part of the olfactory tubercle), Put and Cau (Putamen

1242 and Caudate, derivatives of the dorsal striatum, DSt), Vst, LSt (Ventral Striatum and 
1243 Lateral Striatal Stripe, derivatives of the Ventral Striatum), Ica (intercalated nuclei of the

1244 amygdala), $\mathrm{CeC}$ (capsular part of the central amygdala), ASt (amygdalo-striatal

1245 transitional area), BSTpal (pallidal part of bed nucleus stria terminalis), GPe (external

1246 globus pallidus), VPal (ventral pallidum), OTpal (pallidal part of olfactory tubercle), MeA

1247 (medial amygdala), CeM (medial part of the central amygdala), MSe (medial septum,

1248 with cholinergic ChaAT neurons that may have migrated in from another region), DBV

1249 (diagonal band, vertical limb), BSTdg (diagonal part of bed nucleus stria terminalis),

1250 OTdg (diagonal part of olfactory tubercle), VDg (ventral diagonal area, GPi (internal

1251 globus pallidus), DBH (diagonal band, horizontal limb), SI-Bas-MCPO (substantia

1252 innominata, nucleus basalis, magnocellular preoptic complex), AA (anterior amygdala),

1253 CeL (lateral part of the central amygdala), BSTA (amygdalar part of BST), SeCPOA

1254 (septocommissural POA), MnPO (median preoptic nucleus), LPO (lateral preoptic area),

1255 MPO (medial preoptic area). 


\section{$\underline{\text { References }}$}

1257 Alexa, A., Rahnenfuhrer, J., 2020. topGO: Enrichment Analysis for Gene Ontology.

1258

1259

1260

1261

1262

1263

1264

1265

1266

1267

1268

1269

1270

1271

1272

1273

1274

1275

1276

1277

1278

1279

1280

1281

1282

1283

1284

1285

1286

1287

1288
Anderson, S.A., Eisenstat, D.D., Shi, L., Rubenstein, J.L.R., 1997. Interneuron Migration from Basal Forebrain to Neocortex: Dependence on Dlx Genes. Science 278, 474-476. https://doi.org/10.1126/science.278.5337.474

Andrews, S., 2010. FastQC: a quality control tool for high throughput sequence data.

Angerer, P., Haghverdi, L., Büttner, M., Theis, F.J., Marr, C., Buettner, F., 2016. destiny: diffusion maps for large-scale single-cell data in R. Bioinformatics 32, 12411243. https://doi.org/10.1093/bioinformatics/btv715

Armoskus, C., Moreira, D., Bollinger, K., Jimenez, O., Taniguchi, S., Tsai, H.-W., 2014. Identification of sexually dimorphic genes in the neonatal mouse cortex and hippocampus. Brain Res. 1562, 23-38. https://doi.org/10.1016/j.brainres.2014.03.017

Asbreuk, C.H.J., van Schaick, H.S.A., Cox, J.J., Kromkamp, M., Smidt, M.P., Burbach, J.P.H., 2002. The homeobox genes Lhx7 and Gbx1 are expressed in the basal forebrain cholinergic system. Neuroscience 109, 287-298.

Batista-Brito, R., Ward, C., Fishell, G., 2020. The generation of cortical interneurons. Comprehensive Developmental Neuroscience. Patterning Cell Type Specif. Dev. CNS PNS 1, 461-480.

Beccari, L., Marco-Ferreres, R., Bovolenta, P., 2013. The logic of gene regulatory networks in early vertebrate forebrain patterning. Mech. Dev. 130, 95-111. https://doi.org/10.1016/j.mod.2012.10.004

Bedford, L., Walker, R., Kondo, T., van Crüchten, I., King, E.R., Sablitzky, F., 2005. Id4 is required for the correct timing of neural differentiation. Dev. Biol. 280, 386395. https://doi.org/10.1016/j.ydbio.2005.02.001

Borello, U., Cobos, I., Long, J.E., Murre, C., Rubenstein, J.L., 2008. FGF15 promotes neurogenesis and opposes FGF8 function during neocortical development. Neural Develop. 3, 17. https://doi.org/10.1186/1749-8104-3-17

Breiman, L., 2001. Random Forests. Mach. Learn. 45, 5-32. https://doi.org/10.1023/A:1010933404324

Butler, A., Hoffman, P., Smibert, P., Papalexi, E., Satija, R., 2018. Integrating single-cell transcriptomic data across different conditions, technologies, and species. Nat. Biotechnol. 36, 411-420. https://doi.org/10.1038/nbt.4096 
Campbell, P., Reep, R.L., Stoll, M.L., Ophir, A.G., Phelps, S.M., 2009. Conservation and diversity of Foxp2 expression in muroid rodents: Functional implications. J. Comp. Neurol. 512, 84-100. https://doi.org/10.1002/cne.21881

Carlson, M., 2019. org.Mm.eg.db: Genome wide annotation for Mouse.

Chen, L., Chatterjee, M., Li, J.Y.H., 2010. The mouse homeobox gene Gbx2 is required for the development of cholinergic interneurons in the striatum. J. Neurosci. Off. J. Soc. Neurosci. 30, 14824-14834.

Dobin, A., 2013. STAR: ultrafast universal RNA-seq aligner. Bioinformatics 29, 15-21.

Dunham, I., Kundaje, A., Aldred, S.F., Collins, P.J., Davis, C.A., Doyle, F., Epstein, C.B., Frietze, S., Harrow, J., Kaul, R., Khatun, J., Lajoie, B.R., Landt, S.G., Lee, B.-K., Pauli, F., Rosenbloom, K.R., Sabo, P., Safi, A., Sanyal, A., Shoresh, N., Simon, J.M., Song, L., Trinklein, N.D., Altshuler, R.C., Birney, E., Brown, J.B., Cheng, C., Djebali, S., Dong, X., Dunham, I., Ernst, J., Furey, T.S., Gerstein, M., Giardine, B., Greven, M., Hardison, R.C., Harris, R.S., Herrero, J., Hoffman, M.M., Iyer, S., Kellis, M., Khatun, J., Kheradpour, P., Kundaje, A., Lassmann, T., Li, Q., Lin, X., Marinov, G.K., Merkel, A., Mortazavi, A., Parker, S.C.J., Reddy, T.E., Rozowsky, J., Schlesinger, F., Thurman, R.E., Wang, J., Ward, L.D., Whitfield, T.W., Wilder, S.P., Wu, W., Xi, H.S., Yip, K.Y., Zhuang, J., Bernstein, B.E., Birney, E., Dunham, I., Green, E.D., Gunter, C., Snyder, M., Pazin, M.J., Lowdon, R.F., Dillon, L.A.L., Adams, L.B., Kelly, C.J., Zhang, J., Wexler, J.R., Green, E.D., Good, P.J., Feingold, E.A., Bernstein, B.E., Birney, E., Crawford, G.E., Dekker, J., Elnitski, L., Farnham, P.J., Gerstein, M., Giddings, M.C., Gingeras, T.R., Green, E.D., Guigó, R., Hardison, R.C., Hubbard, T.J., Kellis, M., Kent, W.J., Lieb, J.D., Margulies, E.H., Myers, R.M., Snyder, M., Stamatoyannopoulos, J.A., Tenenbaum, S.A., Weng, Z., White, K.P., Wold, B., Khatun, J., Yu, Y., Wrobel, J., Risk, B.A., Gunawardena, H.P., Kuiper, H.C., Maier, C.W., Xie, L., Chen, X., Giddings, M.C., Bernstein, B.E., Epstein, C.B., Shoresh, N., Ernst, J., Kheradpour, P., Mikkelsen, T.S., Gillespie, S., Goren, A., Ram, O., Zhang, X., Wang, L., Issner, R., Coyne, M.J., Durham, T., Ku, M., Truong, T., Ward, L.D., Altshuler, R.C., Eaton, M.L., Kellis, M., Djebali, S., Davis, C.A., Merkel, A., Dobin, A., Lassmann, T., Mortazavi, A., Tanzer, A., Lagarde, J., Lin, W., Schlesinger, F., Xue, C., Marinov, G.K., Khatun, J., Williams, B.A., Zaleski, C., Rozowsky, J., Röder, M., Kokocinski, F., Abdelhamid, R.F., Alioto, T., Antoshechkin, I., Baer, M.T., Batut, P., Bell, I., Bell, K., Chakrabortty, S., Chen, X., Chrast, J., Curado, J., Derrien, T., Drenkow, J., Dumais, E., Dumais, J., Duttagupta, R., Fastuca, M., Fejes-Toth, K., Ferreira, P., Foissac, S., Fullwood, M.J., Gao, H., Gonzalez, D., Gordon, A., Gunawardena, H.P., Howald, C., Jha, S., Johnson, R., Kapranov, P., King, B., Kingswood, C., Li, G., Luo, O.J., Park, E., Preall, J.B., Presaud, K., Ribeca, P., Risk, B.A., Robyr, D., Ruan, X., Sammeth, M., Sandhu, K.S., Schaeffer, L., See, L.-H., Shahab, A., Skancke, J., Suzuki, A.M., Takahashi, H., Tilgner, H., Trout, D., Walters, N., Wang, H., Wrobel, J., Yu, Y., Hayashizaki, Y., Harrow, J., Gerstein, M., Hubbard, T.J., 
Reymond, A., Antonarakis, S.E., Hannon, G.J., Giddings, M.C., Ruan, Y., Wold, B., Carninci, P., Guigó, R., Gingeras, T.R., Rosenbloom, K.R., Sloan, C.A., Learned, K., Malladi, V.S., Wong, M.C., Barber, G.P., Cline, M.S., Dreszer, T.R., Heitner, S.G., Karolchik, D., Kent, W.J., Kirkup, V.M., Meyer, L.R., Long, J.C., Maddren, M., Raney, B.J., Furey, T.S., Song, L., Grasfeder, L.L., Giresi, P.G., Lee, B.-K., Battenhouse, A., Sheffield, N.C., Simon, J.M., Showers, K.A., Safi, A., London, D., Bhinge, A.A., Shestak, C., Schaner, M.R., Ki Kim, S., Zhang, Z.Z., Mieczkowski, P.A., Mieczkowska, J.O., Liu, Z., McDaniell, R.M., Ni, Y., Rashid, N.U., Kim, M.J., Adar, S., Zhang, Z., Wang, T., Winter, D., Keefe, D., Birney, E., Iyer, V.R., Lieb, J.D., Crawford, G.E., Li, G., Sandhu, K.S., Zheng, M., Wang, P., Luo, O.J., Shahab, A., Fullwood, M.J., Ruan, X., Ruan, Y., Myers, R.M., Pauli, F., Williams, B.A., Gertz, J., Marinov, G.K., Reddy, T.E., Vielmetter, J., Partridge, E., Trout, D., Varley, K.E., Gasper, C., The ENCODE Project Consortium, Overall coordination (data analysis coordination), Data production leads (data production), Lead analysts (data analysis), Writing group, NHGRI project management (scientific management), Principal investigators (steering committee), Boise State University and University of North Carolina at Chapel Hill Proteomics groups (data production and analysis), Broad Institute Group (data production and analysis), Cold Spring Harbor, U. of G., Center for Genomic Regulation, Barcelona, RIKEN, Sanger Institute, University of Lausanne, Genome Institute of Singapore group (data production and analysis), Data coordination center at UC Santa Cruz (production data coordination), Duke University, E., University of Texas, Austin, University of North Carolina-Chapel Hill group (data production and analysis), Genome Institute of Singapore group (data production and analysis), HudsonAlpha Institute, C., UC Irvine, Stanford group (data production and analysis), 2012. An integrated encyclopedia of DNA elements in the human genome. Nature 489, 57-74. https://doi.org/10.1038/nature11247

Eisenstat, D.D., Liu, J.K., Mione, M., Zhong, W., Yu, G., Anderson, S.A., Ghattas, I., Puelles, L., Rubenstein, J.L.R., 1999. DLX-1, DLX-2, and DLX-5 expression define distinct stages of basal forebrain differentiation. J. Comp. Neurol. 414, 217-237. https://doi.org/10.1002/(SICl)1096-9861(19991115)414:2<217::AIDCNE6>3.0.CO;2-I

Elshatory, Y., Gan, L., 2008. The LIM-homeobox gene Islet-1 is required for the development of restricted forebrain cholinergic neurons. J. Neurosci. Off. J. Soc. Neurosci. 28, 3291-3297.

Feng, L., Hatten, M.E., Heintz, N., 1994. Brain lipid-binding protein (BLBP): A novel signaling system in the developing mammalian CNS. Neuron 12, 895-908. https://doi.org/10.1016/0896-6273(94)90341-7

Flames, N., Pla, R., Gelman, D.M., Rubenstein, J.L.R., Puelles, L., Marín, O., 2007. Delineation of multiple subpallial progenitor domains by the combinatorial 
1372

1373

1374

1375

1376

1377

1378

1379

1380

1381

1382

1383

1384

1385

1386

1387

1388

1389

1390

1391

1392

1393

1394

1395

1396

1397

1398

1399

1400

1401

1402

1403

1404

1405

expression of transcriptional codes. J. Neurosci. Off. J. Soc. Neurosci. 27, 96829695.

Flandin, P., Kimura, S., Rubenstein, J.L.R., 2010. The progenitor zone of the ventral medial ganglionic eminence requires Nkx2-1 to generate most of the globus pallidus but few neocortical interneurons. J. Neurosci. Off. J. Soc. Neurosci. 30, 2812-2823.

Fragkouli, A., Wijk, N.V. van, Lopes, R., Kessaris, N., Pachnis, V., 2009. LIM homeodomain transcription factor-dependent specification of bipotential MGE progenitors into cholinergic and GABAergic striatal interneurons. Development 136, 3841-3851. https://doi.org/10.1242/dev.038083

Hafemeister, C., Satija, R., 2019. Normalization and variance stabilization of single-cell RNA-seq data using regularized negative binomial regression. Genome Biol. 20, 296. https://doi.org/10.1186/s13059-019-1874-1

Haubensak, W., Attardo, A., Denk, W., Huttner, W.B., 2004. Neurons arise in the basal neuroepithelium of the early mammalian telencephalon: A major site of neurogenesis. Proc. Natl. Acad. Sci. 101, 3196-3201. https://doi.org/10.1073/pnas.0308600100

Hoch, R.V., Clarke, J.A., Rubenstein, J.L.R., 2015a. Fgf signaling controls the telencephalic distribution of Fgf-expressing progenitors generated in the rostral patterning center. Neural Develop. 10, 8-15.

Hoch, R.V., Lindtner, S., Price, J.D., Rubenstein, J.L.R., 2015b. OTX2 Transcription Factor Controls Regional Patterning within the Medial Ganglionic Eminence and Regional Identity of the Septum. Cell Rep. 12, 482-494.

Hu, J.S., Vogt, D., Lindtner, S., Sandberg, M., Silberberg, S.N., Rubenstein, J.L.R., 2017. Coup-TF1 and Coup-TF2 control subtype and laminar identity of MGEderived neocortical interneurons. Dev. Camb. Engl. 144, 2837-2851.

Inoue, T., Ota, M., Ogawa, M., Mikoshiba, K., Aruga, J., 2007. Zic1 and Zic3 Regulate Medial Forebrain Development through Expansion of Neuronal Progenitors. J. Neurosci. 27, 5461-5473. https://doi.org/10.1523/JNEUROSCI.4046-06.2007

J.L.R., R., Campbell, K., 2020. Neurogenesis in the basal ganglia. Comprehensive Developmental Neuroscience. Patterning Cell Type Specif. Dev. CNS PNS 1, 399-403.

Kageyama, R., Ohtsuka, T., Kobayashi, T., 2008. Roles of Hes genes in neural development. Dev. Growth Differ. 50 Suppl 1, S97-103. 
1406

1407

1408

1409

1410

1411

1412

1413

1414

1415

1416

1417

1418

1419

1420

1421

1422

1423

1424

1425

1426

1427

1428

1429

1430

1431

1432

1433

1434

1435

1436

1437

1438

1439

1440

1441

1442

1443

1444

Kessaris, N., Magno, L., Rubin, A.N., Oliveira, M.G., 2014. Genetic programs controlling cortical interneuron fate. Curr. Opin. Neurobiol., SI: Inhibition: Synapses, Neurons and Circuits 26, 79-87. https://doi.org/10.1016/j.conb.2013.12.012

Kolde, R., 2015. pheatmap: Pretty heatmaps.

Kowalczyk, M.S., Tirosh, I., Heckl, D., Rao, T.N., Dixit, A., Haas, B.J., Schneider, R.K., Wagers, A.J., Ebert, B.L., Regev, A., 2015. Single-cell RNA-seq reveals changes in cell cycle and differentiation programs upon aging of hematopoietic stem cells. Genome Res. 25, 1860-1872. https://doi.org/10.1101/gr.192237.115

Lein, E.S., Hawrylycz, M.J., Ao, N., Ayres, M., Bensinger, A., Bernard, A., Boe, A.F., Boguski, M.S., Brockway, K.S., Byrnes, E.J., Chen, Lin, Chen, Li, Chen, T.-M., Chi Chin, M., Chong, J., Crook, B.E., Czaplinska, A., Dang, C.N., Datta, S., Dee, N.R., Desaki, A.L., Desta, T., Diep, E., Dolbeare, T.A., Donelan, M.J., Dong, H.W., Dougherty, J.G., Duncan, B.J., Ebbert, A.J., Eichele, G., Estin, L.K., Faber, C., Facer, B.A., Fields, R., Fischer, S.R., Fliss, T.P., Frensley, C., Gates, S.N., Glattfelder, K.J., Halverson, K.R., Hart, M.R., Hohmann, J.G., Howell, M.P., Jeung, D.P., Johnson, R.A., Karr, P.T., Kawal, R., Kidney, J.M., Knapik, R.H., Kuan, C.L., Lake, J.H., Laramee, A.R., Larsen, K.D., Lau, C., Lemon, T.A., Liang, A.J., Liu, Y., Luong, L.T., Michaels, J., Morgan, J.J., Morgan, R.J., Mortrud, M.T., Mosqueda, N.F., Ng, L.L., Ng, R., Orta, G.J., Overly, C.C., Pak, T.H., Parry, S.E., Pathak, S.D., Pearson, O.C., Puchalski, R.B., Riley, Z.L., Rockett, H.R., Rowland, S.A., Royall, J.J., Ruiz, M.J., Sarno, N.R., Schaffnit, K., Shapovalova, N.V., Sivisay, T., Slaughterbeck, C.R., Smith, S.C., Smith, K.A., Smith, B.I., Sodt, A.J., Stewart, N.N., Stumpf, K.-R., Sunkin, S.M., Sutram, M., Tam, A., Teemer, C.D., Thaller, C., Thompson, C.L., Varnam, L.R., Visel, A., Whitlock, R.M., Wohnoutka, P.E., Wolkey, C.K., Wong, V.Y., Wood, M., Yaylaoglu, M.B., Young, R.C., Youngstrom, B.L., Feng Yuan, X., Zhang, B., Zwingman, T.A., Jones, A.R., 2007. Genome-wide atlas of gene expression in the adult mouse brain. Nature 445, 168-176. https://doi.org/10.1038/nature05453

Levine, M., 2008. A systems view of Drosophila segmentation. Genome Biol. 9, 207. https://doi.org/10.1186/gb-2008-9-2-207

Liao, Y., Smyth, G.K., Shi, W., 2014. featureCounts: an efficient general purpose program for assigning sequence reads to genomic features. Bioinformatics 30 , 923-930.

Lim, L., Mi, D., Llorca, A., Marín, O., 2018. Development and Functional Diversification of Cortical Interneurons. Neuron 100, 294-313. https://doi.org/10.1016/j.neuron.2018.10.009

Lindtner, S., Catta-Preta, R., Tian, H., Su-Feher, L., Price, J.D., Dickel, D.E., Greiner, V., Silberberg, S.N., McKinsey, G.L., McManus, M.T., Pennacchio, L.A., Visel, A., Nord, A.S., Rubenstein, J.L.R., 2019. Genomic Resolution of DLX-Orchestrated 
Transcriptional Circuits Driving Development of Forebrain GABAergic Neurons. Cell Rep. 28, 2048-2063.e8. https://doi.org/10.1016/j.celrep.2019.07.022

Long, J.E., Garel, S., Alvarez-Dolado, M., Yoshikawa, K., Osumi, N., Alvarez-Buylla, A., Rubenstein, J.L.R., 2007. Dlx-Dependent and -Independent Regulation of Olfactory Bulb Interneuron Differentiation. J. Neurosci. 27, 3230-3243. https://doi.org/10.1523/JNEUROSCI.5265-06.2007

Long, J.E., Swan, C., Liang, W.S., Cobos, I., Potter, G.B., Rubenstein, J.L.R., 2009. DIx1\&2 and Mash1 transcription factors control striatal patterning and differentiation through parallel and overlapping pathways. J. Comp. Neurol. 512, 556-572.

Madisen, L., Zwingman, T.A., Sunkin, S.M., Oh, S.W., Zariwala, H.A., Gu, H., Ng, L.L., Palmiter, R.D., Hawrylycz, M.J., Jones, A.R., Lein, E.S., Zeng, H., 2010. A robust and high-throughput Cre reporting and characterization system for the whole mouse brain. Nat. Neurosci. 13, 133-140.

Magno, L., Barry, C., Schmidt-Hieber, C., Theodotou, P., Häusser, M., Kessaris, N., 2017. NKX2-1 Is Required in the Embryonic Septum for Cholinergic System Development, Learning, and Memory. Cell Rep. 20, 1572-1584.

Marín, O., Anderson, S.A., Rubenstein, J.L.R., 2000. Origin and Molecular Specification of Striatal Interneurons. J. Neurosci. 20, 6063-6076. https://doi.org/10.1523/JNEUROSCI.20-16-06063.2000

Mayer, C., Hafemeister, C., Bandler, R.C., Machold, R., Batista Brito, R., Jaglin, X., Allaway, K., Butler, A., Fishell, G., Satija, R., 2018. Developmental diversification of cortical inhibitory interneurons. Nature 555, 457-462. https://doi.org/10.1038/nature25999

McGinnis, C.S., Patterson, D.M., Winkler, J., Conrad, D.N., Hein, M.Y., Srivastava, V., Hu, J.L., Murrow, L.M., Weissman, J.S., Werb, Z., Chow, E.D., Gartner, Z.J., 2019. MULTI-seq: sample multiplexing for single-cell RNA sequencing using lipid-tagged indices. Nat. Methods 16, 619-626.

McGregor, M.M., McKinsey, G.L., Girasole, A.E., Bair-Marshall, C.J., Rubenstein, J.L.R., Nelson, A.B., 2019. Functionally Distinct Connectivity of Developmentally Targeted Striosome Neurons. Cell Rep. 29, 1419-1428.e5. https://doi.org/10.1016/j.celrep.2019.09.076

Mclnnes, L., Healy, J., Saul, N., Großberger, L., 2018. UMAP: Uniform Manifold Approximation and Projection. J. Open Source Softw. 3, 861. https://doi.org/10.21105/joss.00861 
Mckinsey, G.L., Lindtner, S., Trzcinski, B., Visel, A., Pennacchio, L.A., Huylebroeck, D., Higashi, Y., Rubenstein, J.L.R., 2013. Dlx1\&2-dependent expression of Zfhx1b (Sip1, Zeb2) regulates the fate switch between cortical and striatal interneurons. Neuron 77, 83-98.

Mi, D., Li, Z., Lim, L., Li, M., Moissidis, M., Yang, Y., Gao, T., Hu, T.X., Pratt, T., Price, D.J., Sestan, N., Marín, O., 2018. Early emergence of cortical interneuron diversity in the mouse embryo. Science $360,81-85$. https://doi.org/10.1126/science.aar6821

Nóbrega-Pereira, S., Gelman, D., Bartolini, G., Pla, R., Pierani, A., Marín, O., 2010. Origin and Molecular Specification of Globus Pallidus Neurons. J. Neurosci. 30, 2824-2834. https://doi.org/10.1523/JNEUROSCI.4023-09.2010

Nord, A.S., 2015. Learning about mammalian gene regulation from functional enhancer assays in the mouse. Genomics, Recent advances in functional assays of transcriptional enhancers 106, 178-184. https://doi.org/10.1016/j.ygeno.2015.06.008

Nord, A.S., 2013. Rapid and pervasive changes in genome-wide enhancer usage during mammalian development. Cell 155, 1521-1531.

Pai, E.L.-L., Vogt, D., Clemente-Perez, A., Mckinsey, G.L., Cho, F.S., Hu, J.S., Wimer, M., Paul, A., Fazel Darbandi, S., Pla, R., Nowakowski, T.J., Goodrich, L.V., Paz, J.T., Rubenstein, J.L.R., 2019. Mafb and c-Maf Have Prenatal Compensatory and Postnatal Antagonistic Roles in Cortical Interneuron Fate and Function. Cell Rep. 26, 1157-1173.e5.

Pattabiraman, K., Golonzhka, O., Lindtner, S., Nord, A.S., Taher, L., Hoch, R., Silberberg, S.N., Zhang, D., Chen, B., Zeng, H., Pennacchio, L.A., Puelles, L., Visel, A., Rubenstein, J.L.R., 2014. Transcriptional Regulation of Enhancers Active in Protodomains of the Developing Cerebral Cortex. Neuron 82, 9891003. https://doi.org/10.1016/j.neuron.2014.04.014 control neuronal versus oligodendroglial cell fate acquisition in the developing forebrain. Neuron 55, 417-433.

1510 Picard Toolkit, 2019. . Broad Institute, GitHub Repository. molecularly distinct cell populations in the embryonic mouse forebrain. J. Neurosci. 14, 6370-6383. https://doi.org/10.1523/JNEUROSCI.14-11- 
1516

1517

1518

1519

1520

1521

1522

1523

1524

1525

1526

1527

1528

1529

1530

1531

1532

1533

1534

1535

1536

1537

1538

1539

1540

1541

1542

1543

1544

1545

1546

1547

1548

1549

1550

1551

Preibisch, S., Saalfeld, S., Tomancak, P., 2009. Globally optimal stitching of tiled 3D microscopic image acquisitions. Bioinformatics 25, 1463-1465.

Puelles, L., Harrison, M., Paxinos, G., Watson, C., 2013. A developmental ontology for the mammalian brain based on the prosomeric model. Trends Neurosci. 36, 570578. https://doi.org/10.1016/j.tins.2013.06.004

Puelles, L., Morales-Delgado, N., Merchán, P., Castro-Robles, B., Martínez-de-la-Torre, M., Díaz, C., Ferran, J.L., 2016. Radial and tangential migration of telencephalic somatostatin neurons originated from the mouse diagonal area. Brain Struct. Funct. 221, 3027-3065.

Reddington, J.P., Garfield, D.A., Sigalova, O.M., Karabacak Calviello, A., MarcoFerreres, R., Girardot, C., Viales, R.R., Degner, J.F., Ohler, U., Furlong, E.E.M., 2020. Lineage-Resolved Enhancer and Promoter Usage during a Time Course of Embryogenesis. Dev. Cell 55, 648-664.e9. https://doi.org/10.1016/j.devcel.2020.10.009

Roychoudhury, K., Salomone, J., Qin, S., Cain, B., Adam, M., Potter, S.S., Nakafuku, M., Gebelein, B., Campbell, K., 2020. Physical interactions between Gsx2 and Ascl1 balance progenitor expansion versus neurogenesis in the mouse lateral ganglionic eminence. Dev. Camb. Engl. 147, dev185348.

Rubin, A.N., Alfonsi, F., Humphreys, M.P., Choi, C.K.P., Rocha, S.F., Kessaris, N., 2010. The Germinal Zones of the Basal Ganglia But Not the Septum Generate GABAergic Interneurons for the Cortex. J. Neurosci. 30, 12050-12062. https://doi.org/10.1523/JNEUROSCI.6178-09.2010

Sanchez-Ortiz, E., Yui, D., Song, D., Li, Y., Rubenstein, J.L., Reichardt, L.F., Parada, L.F., 2012. TrkA Gene Ablation in Basal Forebrain Results in Dysfunction of the Cholinergic Circuitry. J. Neurosci. 32, 4065-4079. https://doi.org/10.1523/JNEUROSCI.6314-11.2012

Sandberg, M., Flandin, P., Silberberg, S., Su-Feher, L., Price, J.D., Hu, J.S., Kim, C., Visel, A., Nord, A.S., Rubenstein, J.L.R., 2016. Transcriptional Networks Controlled by NKX2-1 in the Development of Forebrain GABAergic Neurons. Neuron 91, 1260-1275. https://doi.org/10.1016/j.neuron.2016.08.020

Silberberg, S.N., Taher, L., Lindtner, S., Sandberg, M., Nord, A.S., Vogt, D., Mckinsey, G.L., Hoch, R., Pattabiraman, K., Zhang, D., Ferran, J.L., Rajkovic, A., Golonzhka, O., Kim, C., Zeng, H., Puelles, L., Visel, A., Rubenstein, J.L.R., 2016. Subpallial Enhancer Transgenic Lines: a Data and Tool Resource to Study Transcriptional Regulation of GABAergic Cell Fate. Neuron 92, 59-74. https://doi.org/10.1016/j.neuron.2016.09.027 
1552

1553

1554

1555

1556

1557

1558

1559

1560

1561

1562

1563

1564

1565

1566

1567

1568

1569

1570

1571

1572

1573

1574

1575

1576

1577

1578

1579

1580

1581

1582

1583
Stuart, T., Butler, A., Hoffman, P., Hafemeister, C., Papalexi, E., Mauck, W.M., Hao, Y., Stoeckius, M., Smibert, P., Satija, R., 2019. Comprehensive Integration of SingleCell Data. Cell 177, 1888-1902.e21. https://doi.org/10.1016/j.cell.2019.05.031

Visel, A., Taher, L., Girgis, H., May, D., Golonzhka, O., Hoch, R.V., McKinsey, G.L., Pattabiraman, K., Silberberg, S.N., Blow, M.J., Hansen, D.V., Nord, A.S., Akiyama, J.A., Holt, A., Hosseini, R., Phouanenavong, S., Plajzer-Frick, I., Shoukry, M., Afzal, V., Kaplan, T., Kriegstein, A.R., Rubin, E.M., Ovcharenko, I., Pennacchio, L.A., Rubenstein, J.L.R., 2013. A High-Resolution Enhancer Atlas of the Developing Telencephalon. Cell 152, 895-908. https://doi.org/10.1016/j.cell.2012.12.041

Wang, L., Wang, S., Li, W., 2012. RSeQC: quality control of RNA-seq experiments. Bioinformatics 28, 2184-2185.

Yun, K., Mantani, A., Garel, S., Rubenstein, J., Israel, M.A., 2004. Id4 regulates neural progenitor proliferation and differentiation in vivo. Development 131, 5441-5448. https://doi.org/10.1242/dev.01430

Yuzwa, S.A., Borrett, M.J., Innes, B.T., Voronova, A., Ketela, T., Kaplan, D.R., Bader, G.D., Miller, F.D., 2017. Developmental Emergence of Adult Neural Stem Cells as Revealed by Single-Cell Transcriptional Profiling. Cell Rep. 21, 3970-3986.

Zeisel, A., Hochgerner, H., Lönnerberg, P., Johnsson, A., Memic, F., van der Zwan, J., Häring, M., Braun, E., Borm, L.E., La Manno, G., Codeluppi, S., Furlan, A., Lee, K., Skene, N., Harris, K.D., Hjerling-Leffler, J., Arenas, E., Ernfors, P., Marklund, U., Linnarsson, S., 2018. Molecular Architecture of the Mouse Nervous System. Cell 174, 999-1014.e22. https://doi.org/10.1016/j.cell.2018.06.021

Zheng, G.X.Y., Terry, J.M., Belgrader, P., Ryvkin, P., Bent, Z.W., Wilson, R., Ziraldo, S.B., Wheeler, T.D., McDermott, G.P., Zhu, J., Gregory, M.T., Shuga, J., Montesclaros, L., Underwood, J.G., Masquelier, D.A., Nishimura, S.Y., SchnallLevin, M., Wyatt, P.W., Hindson, C.M., Bharadwaj, R., Wong, A., Ness, K.D., Beppu, L.W., Deeg, H.J., McFarland, C., Loeb, K.R., Valente, W.J., Ericson, N.G., Stevens, E.A., Radich, J.P., Mikkelsen, T.S., Hindson, B.J., Bielas, J.H., 2017. Massively parallel digital transcriptional profiling of single cells. Nat. Commun. 8, 14049. https://doi.org/10.1038/ncomms14049 